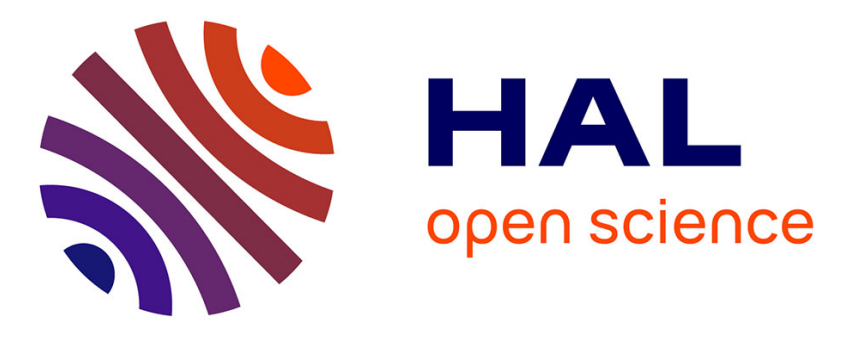

\title{
Global and Local Deadlock Freedom in BIP
}

Paul C Attie, Saddek Bensalem, Marius Bozga, Mohamad Jaber, Joseph

Sifakis, Fadi Zaraket

\section{To cite this version:}

Paul C Attie, Saddek Bensalem, Marius Bozga, Mohamad Jaber, Joseph Sifakis, et al.. Global and Local Deadlock Freedom in BIP. ACM Transactions on Software Engineering and Methodology, 2018, 26 (3), pp.1 - 48. 10.1145/3152910 . hal-01865301

\section{HAL Id: hal-01865301 \\ https://hal.science/hal-01865301}

Submitted on 31 Aug 2018

HAL is a multi-disciplinary open access archive for the deposit and dissemination of scientific research documents, whether they are published or not. The documents may come from teaching and research institutions in France or abroad, or from public or private research centers.
L'archive ouverte pluridisciplinaire HAL, est destinée au dépôt et à la diffusion de documents scientifiques de niveau recherche, publiés ou non, émanant des établissements d'enseignement et de recherche français ou étrangers, des laboratoires publics ou privés. 


\title{
Global and Local Deadlock Freedom in BIP
}

\author{
PAUL C ATTIE, American University of Beirut, Lebanon \\ SADDEK BENSALEM, UJF-Grenoble 1 / CNRS VERIMAG, France \\ MARIUS BOZGA, UJF-Grenoble 1 / CNRS VERIMAG, France \\ MOHAMAD JABER, American University of Beirut, Lebanon \\ JOSEPH SIFAKIS, Ecole Polytechnique Federale Lausanne, Switzerland \\ FADI A ZARAKET, American University of Beirut, Lebanon
}

CCS Concepts: • Theory of computation $\rightarrow$ Program verification; $\bullet$ Software and its engineering $\rightarrow$
Deadlocks; Model checking; Formal software verification; State systems; Synchronization;

Additional Key Words and Phrases: Alternation, Completeness, Nondeterminism

ACM Reference Format:

Paul C Attie, Saddek Bensalem, Marius Bozga, Mohamad Jaber, Joseph Sifakis, and Fadi A Zaraket. 2010. Global and Local Deadlock Freedom in BIP. ACM Trans. Softw. Eng. Methodol. 9, 4, Article 39 (March 2010), 49 pages. https://doi.org/0000001.0000001

\section{INTRODUCTION}

Deadlock freedom is a crucial property of concurrent and distributed systems. With increasing system complexity, the challenge of assuring deadlock freedom and other correctness properties becomes even greater. In contrast to the alternatives of (1) deadlock detection and recovery, and (2) deadlock avoidance, we advocate deadlock prevention: design the system so that deadlocks do not occur.

Deciding deadlock freedom of finite-state concurrent programs is PSPACE-complete, in general [26, chapter 19]. To achieve tractability, we present a criterion for deadlock-freedom that is evaluated by model-checking a set of subsystems of the overall system. If the subsystems are small, the criterion can be checked quickly. The criterion is sound (if true, it implies deadlock-freedom) but not complete (if false, then it yields no information about deadlock). If the subsystems are larger, then our criterion becomes more "accurate": roughly speaking, there is less possibility for the criterion to evaluate to false when the system is actually deadlock-free. In the limit, when the set of subsystems includes the entire system itself, our criterion is complete, so that evaluation to false implies that the system is actually deadlock-prone. Hence, our criterion only fails to resolve the

Authors' addresses: Paul C Attie, American University of Beirut, PO Box 11-0236, Riad El Solh, Beirut, 1107 2020, Lebanon, paul.attie@aub.edu.lb; Saddek Bensalem, UJF-Grenoble 1 / CNRS VERIMAG, UMR 5104, Grenoble, F-38041, France, Saddek. Bensalem@imag.fr; Marius Bozga, UJF-Grenoble 1 / CNRS VERIMAG, UMR 5104, Grenoble, F-38041, France, Marius. Bozga@imag.fr; Mohamad Jaber, American University of Beirut, PO Box 11-0236, Riad El Solh, Beirut, 1107 2020, Lebanon, mj54@aub.edu.lb; Joseph Sifakis, Ecole Polytechnique Federale Lausanne, Route Cantonale, Lausanne, 1015, Switzerland, joseph.sifakis@imag.fr; Fadi A Zaraket, American University of Beirut, PO Box 11-0236, Riad El Solh, Beirut, 11072020 Lebanon, fz11@aub.edu.lb.

Permission to make digital or hard copies of all or part of this work for personal or classroom use is granted without fee provided that copies are not made or distributed for profit or commercial advantage and that copies bear this notice and the full citation on the first page. Copyrights for components of this work owned by others than the author(s) must be honored. Abstracting with credit is permitted. To copy otherwise, or republish, to post on servers or to redistribute to lists, requires prior specific permission and/or a fee. Request permissions from permissions@acm.org.

(C) 2009 Copyright held by the owner/author(s). Publication rights licensed to Association for Computing Machinery.

1049-331X/2010/3-ART39 \$15.00

https://doi.org/0000001.0000001 
question of deadlock-freedom when it's evaluation exhausts available computational resources, because the subsystems being checked have become too large, and state-explosion has set in.

Our method thus combines the possibility of fast response together with theoretical completeness. All deadlock-freedom checks given in the literature to date are, to our knowledge, incomplete in principle, and so remain incomplete even if unlimited computational resources are available. Hence these criteria could fail to resolve deadlock freedom for theoretical reasons, as well as for lack of computational resources. The reason for this incompleteness is that existing criteria all characterize deadlock by the occurrence of a wait-for cycle, e.g., as stated by [3], discussion of related work:

All these methods were designed, to some extent, around the principle that under reasonable assumptions about the system, any deadlock state would contain a proper cycle of ungranted requests.

In a model of concurrency which includes choice of actions (e.g., BIP, CSP, I/O automata, CCS), a wait-for cycle is an incomplete characterization of deadlock, since a process can be in a wait-for cycle, but not deadlocked, due to having a choice of interaction with another process not in the wait-for cycle (see Figure 5 below).

Our method, in contrast, characterizes deadlock by the occurrence of a supercycle $[7,8]$, which, very roughly, is the AND-OR analogue of a wait-for cycle: a subset of processes constitutes a supercycle $S C$ iff every possible action of every process in $S C$ is blocked by another process in $S C$. We show that supercycles are a sound and complete characterization of deadlock: a system is deadlock-prone iff a supercycle can arise in some reachable state. We then present our criterion, which prevents the occurrence of supercycles in reachable states of the system. We first present a "global" version of our criterion, which is both sound and complete w.r.t. absence of supercycles, and then a "local" version, which is sound w.r.t. absence of supercycles, and can be evaluated over small subsystems.

Our criterion guarantees freedom from local (and therefore global) deadlock. A local deadlock occurs when a subsystem is deadlocked while the rest of the system can execute. Other criteria in the literature [2, 3, 13, 17, 21, 23, 24, 29] guarantee only global deadlock freedom.

This paper significantly extends a preliminary conference version [6] as follows: (1) we present an "AND-OR" criterion for deadlock-freedom, which exploits the AND-OR structure of supercycles, and is therefore complete for deadlock-freedom in the limit, while our preliminary work [6] gives a "linear" criterion, which is a special case in which the AND-OR structure is ignored, and (2) experimental results show that the new criterion is more efficient in practice, and also succeeds in cases where the linear criterion fails. We therefore have the best of both worlds: early stopping, and therefore efficient verification of deadlock-freedom, in many cases, together with theoretical completeness. Our criterion is, to the best of our knowledge, the first criterion that is sound and complete for global and local deadlock-freedom in concurrent programs with nondeterministic local choice, i.e., a process can nondeterministically choose among enabled actions.

We present experimental results for dining philosophers and for a multi-token based resource allocation system, which generalizes Milner's token based scheduler [25]. These show that our method compares favorably with existing approaches.

Section 2 presents BIP [14]. Section 3 characterizes local and global deadlocks as the occurrence of a pattern of wait-for edges called a supercycle (see discussion above). Section 4 considers "global" supercycles, i.e., those that occur in the overall system being considered, characterizes these as the greatest fixpoint of a "blocking" operator, and presents some structural properties of supercycles. Section 5 presents global conditions for the prevention of the formation of supercycles. Global means that these conditions are evaluated in the entire system. Section 6 considers "local" supercycles, i.e., those that occur in a given subsystem of the overall system being considered. 
These are characterized as the greatest fixpoint of a "local blocking" operator, which pessimistically assumes that nodes on the boundary of the subsystem are blocked. This pessimistic assumption ensures soundness, at the expense of completeness. Section 7 presents local conditions for the prevention of the formation of supercycles. These can be evaluated in (small) subsystems of the overall system, and are obtained by "projecting" the global conditions onto a subsystem. Section 8 presents the main soundness and completeness results of the paper, and gives the implication relation among our various conditions for deadlock-freedom. Section 9 gives algorithms to evaluate the local conditions, and presents experimental evaluation. Section 10 discusses related work, further work, and concludes.

\section{BIP - BEHAVIOR INTERACTION PRIORITY}

BIP is a component framework for constructing systems by superposing three layers of modeling: Behavior, Interaction, and Priority. A technical treatment of priority is beyond the scope of this paper. Adding priorities never introduces a deadlock, since priority enforces a choice between possible transitions from a state, and deadlock-freedom means that there is at least one transition from every (reachable) state. Hence if a BIP system without priorities is deadlock-free, then the same system with priorities added will also be deadlock-free.

Definition 2.1 (Atomic component). An atomic component $\mathrm{B}_{i}$ is a labeled transition system represented by a triple $\left(Q_{i}, P_{i}, \rightarrow_{i}\right)$ where $Q_{i}$ is a set of states, $P_{i}$ is a set of communication ports, and $\rightarrow_{i} \subseteq Q_{i} \times P_{i} \times Q_{i}$ is a set of transitions, each labeled by some port.

For states $s_{i}, t_{i} \in Q_{i}$ and port $p_{i} \in P_{i}$, write $s_{i} \stackrel{p_{i}}{\rightarrow} t_{i}$, iff $\left(s_{i}, p_{i}, t_{i}\right) \in \rightarrow_{i}$. When $p_{i}$ is clear from the context or not needed, we drop it from the transition and write $s_{i} \rightarrow_{i} t_{i}$. Similarly, $s_{i} \stackrel{p_{i}}{\rightarrow}$ means that there exists $t_{i} \in Q_{i}$ such that $s_{i}{\stackrel{p_{i}}{\rightarrow}}_{i} t_{i}$. In this case, $p_{i}$ is enabled in state $s_{i}$. Ports are used for communication between different components, as discussed below.

Figure 1(a) shows atomic components for a philosopher $P h_{i}$ and a fork $F_{i}$ in dining philosophers. A philosopher $P h_{i}$ that is hungry (in state $h_{i}$ ) can eat by executing get ${ }_{i}$ (to get its forks) and moving to state $e_{i}$ (eating). From $e_{i}, P h_{i}$ releases its forks by executing $p u t_{i}$ (to put down its forks) and moving back to $h_{i}$. Adding the thinking state does not change the deadlock behaviour of the system, since the thinking to hungry transition is internal to $P h_{i}$, and so we omit it. A fork $F_{i}$ is taken by either: (1) the left philosopher (transition $u s e_{i}^{\ell}$ ) and so moves to state $u_{i}^{\ell}$ (used by left philosopher), or (2) the right philosopher (transition $u s e_{i}^{r}$ ) and so moves to state $u_{i}^{r}$ (used by right philosopher) From state $u_{i}^{r}$ (resp. $\left.u_{i}^{\ell}\right), F_{i}$ is released by the right philosopher (resp. left philosopher) and so moves back to state $f_{i}$ (free).

In practice, we describe the transition system using some syntax, e.g., involving local variables (BIP does not have shared variables). We abstract away from issues of syntactic description since we are only interested in enablement of ports and actions. In BIP, the enablement of a port depends only on the local state of a component. In particular, it cannot depend on the state of other components. For example, state $e_{i}$ in atomic component $P h_{i}$ of Figure 1(a) enables port $p u t_{i}$ as there exists a transition from $e_{i}$ labeled with port $p u t_{i}$. Hence, there exists a predicate $e n b_{p_{i}}^{i}$ that holds in state $s_{i}$ of component $\mathrm{B}_{i}$ iff port $p_{i}$ is enabled in $s_{i}$, i.e., $s_{i}\left(e n b_{p_{i}}^{i}\right)=$ true iff $s_{i}{\stackrel{p_{i}}{\rightarrow}}_{i}$.

Definition 2.2 (Interaction). For a given system built from a set of $n$ atomic components $\left\{\mathrm{B}_{i}=\right.$ $\left.\left(Q_{i}, P_{i}, \rightarrow_{i}\right)\right\}_{i=1}^{n}$, we require that their respective sets of ports are pairwise disjoint, i.e., for all $i, j$ such that $i, j \in\{1 . . n\} \wedge i \neq j$, we have $P_{i} \cap P_{j}=\emptyset$. An interaction is a set of ports not containing two or more ports from the same component. That is, for an interaction a we have 

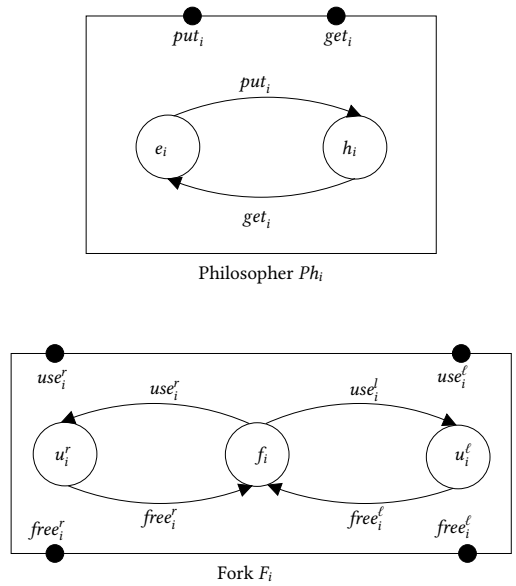

(a) Philosopher $P h_{i}$ and fork $F_{i}$ atomic components.

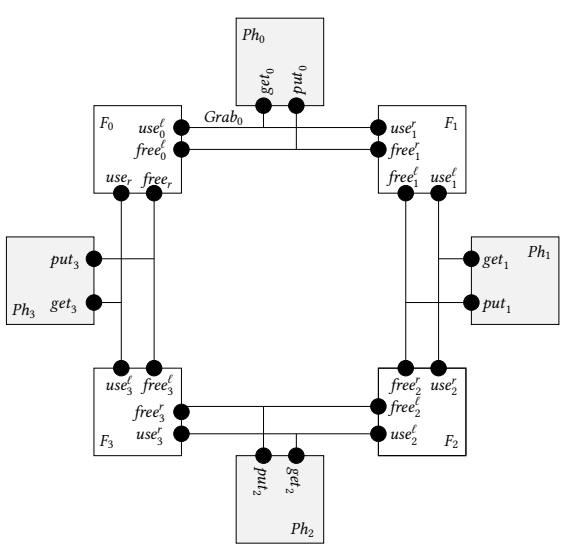

(b) Dining philosophers composite component with four philosophers.

Fig. 1. Dining philosophers.

$\mathrm{a} \subseteq P \wedge\left(\forall i \in\{1 . . n\}:\left|\mathrm{a} \cap P_{i}\right| \leq 1\right)$, where $P=\bigcup_{i=1}^{n} P_{i}$ is the set of all ports in the system. When we write a $=\left\{p_{i}\right\}_{i \in I}$, we assume that $p_{i} \in P_{i}$ for all $i \in I$, where $I \subseteq\{1 . . n\}$.

The Connectors that connect ports in Figure 1(b) illustrate interactions. For example, the interaction $\mathrm{Grab}_{0}=\left\{\right.$ get $_{0}$, use $\left._{0}^{\ell}, u s e_{1}^{r}\right\}$ connects ports get $_{0}, u s e_{0}^{\ell}$, and $u s e_{1}^{r}$ from components $P h_{0}, F_{0}$, and $F_{1}$ respectively, and corresponds to philosopher component $P h_{0}$ acquiring both forks and moving to it's eating state.

Execution of an interaction $\mathrm{a}=\left\{p_{i}\right\}_{i \in I}$ involves all the components which have ports in a. We denote by components (a) the set of atomic components participating in a. Formally, components $(\mathrm{a})=$ $\left\{\mathrm{B}_{i} \mid p_{i} \in \mathrm{a}\right\}$.

Definition 2.3 (Composite component). A composite component (or simply component) $\mathrm{B} \triangleq$ $\gamma\left(\mathrm{B}_{1}, \ldots, \mathrm{B}_{n}\right)$ is defined by a composition operator parameterized by a set of interactions $\gamma \subseteq 2^{P}$. B has a transition system $(Q, \gamma, \rightarrow)$, where $Q=Q_{1} \times \cdots \times Q_{n}$ and $\rightarrow \subseteq Q \times \gamma \times Q$ is the least set of transitions satisfying the rule

$$
\frac{\mathrm{a}=\left\{p_{i}\right\}_{i \in I} \in \gamma \quad \forall i \in I: s_{i}{\stackrel{p_{i}}{\rightarrow}}_{i} t_{i} \quad \forall i \notin I: s_{i}=t_{i}}{\left\langle s_{1}, \ldots, s_{n}\right\rangle \stackrel{\mathrm{a}}{\rightarrow}\left\langle t_{1}, \ldots, t_{n}\right\rangle}
$$

This inference rule says that a composite component $\mathrm{B}=\gamma\left(\mathrm{B}_{1}, \ldots, \mathrm{B}_{n}\right)$ can execute an interaction $\mathrm{a} \in \gamma$, iff for each port $p_{i} \in \mathrm{a}$, the corresponding atomic component $\mathrm{B}_{i}$ can execute a transition labeled with $p_{i}$; the states of components that do not participate in the interaction stay unchanged. Note that interactions are the only means of inter-component communication and synchronization in BIP.

Example 2.4 (Composite component). Figure 1(b) shows a composite component consisting of four philosophers and the four forks between them. Each philosopher $P h_{i}(0 \leq i \leq 3)$ and its two neighboring forks share two interactions: $G r a b_{i}=\left\{\right.$ get $\left._{i}, u e_{i}^{\ell}, u s e_{i+1}^{r}\right\}$ in which the philosopher obtains the forks, and $\operatorname{Rel}_{i}=\left\{\right.$ put $_{i}$, free $_{i}^{\ell}$, free $\left._{i+1}^{r}\right\}$ in which the philosopher releases the forks (addition of indices being modulo 4). 
Definition 2.5 (Interaction enablement). An atomic component $\mathrm{B}_{i}=\left(Q_{i}, P_{i}, \rightarrow_{i}\right)$ enables a port $p_{i} \in P_{i}$ in state $s_{i}$ iff $s_{i} \stackrel{p}{\rightarrow}_{i}$. $\mathrm{B}_{i}$ enables interaction a in state $s_{i}$ iff $s_{i} \stackrel{p}{\rightarrow}_{i}$, where $\left\{p_{i}\right\}=P_{i} \cap$ a is the port of $\mathrm{B}_{i}$ involved in a. That is, $\mathrm{B}_{i}$ enables a in state $s_{i}$ iff $\mathrm{B}_{i}$ enables port a $\cap P_{i}$ in state $s_{i}$.

Recall that $e n b_{p_{i}}^{i}$ denotes the enablement condition for port $p_{i}$ in component $\mathrm{B}_{i}$, that is, en $b_{p_{i}}^{i}$ holds iff $s_{i} \stackrel{p_{i}}{\rightarrow}$, where $s_{i}$ is the current state of $\mathrm{B}_{i}$. Let $e n b_{\mathrm{a}}^{i}$ denote the enablement condition for interaction a in component $\mathrm{B}_{i}$, that is, en $b_{\mathrm{a}}^{i}=e n b_{p_{i}}^{i}$ where $\left\{p_{i}\right\}=\mathrm{a} \cap P_{i}$.

Let $\mathrm{B}=\gamma\left(\mathrm{B}_{1}, \ldots, \mathrm{B}_{n}\right)$ be a composite component, let $s=\left\langle s_{1}, \ldots, s_{n}\right\rangle$ be a state of $\mathrm{B}$, and let $\mathrm{a}=\left\{p_{i}\right\}_{i \in I}$ be an interaction of $\mathrm{B}$. Then $\mathrm{B}$ enables a in $s$ iff every $\mathrm{B}_{i} \in$ components(a) enables a in $s_{i}$, i.e., iff $\bigwedge_{i \in I} e n b_{\mathrm{a}}^{i}$ holds in $s$.

The definition of interaction enablement is a consequence of Definition 2.3. Interaction a being enabled in state $s$ means that executing a is one of the possible transitions that can be taken from $s$

To avoid pathological cases of deadlock due solely to a single component refusing to enable any interaction at all, we assume that every component always enables at least one interaction. Structurally, this means that there is no local state with zero transitions, and every port labeling a transition is part of at least one interaction. Intuitively, component $\mathrm{B}_{i}$ in state $s$ must enable at least one interaction a. However, a requires enablement from all components involved in it to execute. That might not be the case as a may be blocked by another component $\mathrm{B}_{j} \neq \mathrm{B}_{i}$. Therefore the assumption is not enough to guarantee deadlock freedom.

Definition 2.6 (Local enablement assumption). For every component $\mathrm{B}_{i}=\left(Q_{i}, P_{i}, \rightarrow_{i}\right)$, the following holds. In every $s_{i} \in Q_{i}, \mathrm{~B}_{i}$ enables some interaction a.

Definition 2.7 (BIP-system). Let $\mathrm{B}=\gamma\left(\mathrm{B}_{1}, \ldots, \mathrm{B}_{n}\right)$ be a composite component with transition system $(Q, \gamma, \rightarrow)$, and let $Q_{0} \subseteq Q$ be a set of initial states. Then (B, $\left.Q_{0}\right)$ is a BIP system.

Figure 1(b) gives a BIP-system with philosophers initially in state $h$ (hungry) and forks initially in state $f$ (free). To avoid tedious repetition, we fix, for the rest of the paper, an arbitrary BIP-system $\left(\mathrm{B}, Q_{0}\right)$, with $\mathrm{B} \triangleq \gamma\left(\mathrm{B}_{1}, \ldots, \mathrm{B}_{n}\right)$, and transition system $(Q, \gamma, \rightarrow)$.

Definition 2.8 (Execution). Let $\rho=s_{0} \mathrm{a}_{1} s_{1} \ldots s_{j-1} \mathrm{a}_{j} s_{j} \ldots$ be an alternating sequence of states of $\mathrm{B}$ and interactions of $\mathrm{B}$. Then $\rho$ is an execution of (B, $\left.Q_{0}\right)$ iff $(1) s_{0} \in Q_{0}$, and (2) $\forall j>0: s_{j-1} \stackrel{\mathrm{a}_{j}}{\rightarrow} s_{j}$.

Definition 2.9 (Reachable state, transition). A state or transition that occurs in some execution is called reachable. rstates $\left(\mathrm{B}, Q_{0}\right)$ denotes the set of reachable states of $\left(\mathrm{B}, Q_{0}\right)$.

Definition 2.10 (State projection). Let $s=\left\langle s_{1}, \ldots, s_{n}\right\rangle$ be a state of $\left(\mathrm{B}, Q_{0}\right)$. Let $\left\{\mathrm{B}_{i_{1}}, \ldots, \mathrm{B}_{i_{k}}\right\} \subseteq$ $\left\{\mathrm{B}_{1}, \ldots, \mathrm{B}_{n}\right\}$. Then $s\left\lceil\left\{\mathrm{~B}_{i_{1}}, \ldots, \mathrm{B}_{i_{k}}\right\} \triangleq\left\langle s_{i_{1}}, \ldots, s_{i_{k}}\right\rangle\right.$. For a single $\mathrm{B}_{i}$, we write $s\left\lceil\mathrm{~B}_{i}=s_{i}\right.$. We extend state projection to sets of states element-wise.

Definition 2.11 (Subcomponent). Let $\left\{\mathrm{B}_{i_{1}}, \ldots, \mathrm{B}_{i_{k}}\right\} \subseteq\left\{\mathrm{B}_{1}, \ldots, \mathrm{B}_{n}\right\}$. Let $P^{\prime}=P_{i_{1}} \cup \cdots \cup P_{i_{k}}$, i.e., the union of the ports of $\left\{\mathrm{B}_{i_{1}}, \ldots, \mathrm{B}_{i_{k}}\right\}$. Then the subcomponent $\mathrm{B}^{\prime}$ of $\mathrm{B}$ based on $\left\{\mathrm{B}_{i_{1}}, \ldots, \mathrm{B}_{i_{k}}\right\}$ is as follows:

(1) $\gamma^{\prime} \triangleq\left\{a \cap P^{\prime} \mid a \in \gamma \wedge a \cap P^{\prime} \neq \emptyset\right\}$

(2) $\mathrm{B}^{\prime} \triangleq \gamma^{\prime}\left(\mathrm{B}_{i_{1}}, \ldots, \mathrm{B}_{i_{k}}\right)$

That is, $\gamma^{\prime}$ consists of those interactions in $\gamma$ that have at least one participant in $\left\{\mathrm{B}_{i_{1}}, \ldots, \mathrm{B}_{i_{k}}\right\}$, and restricted to the participants in $\left\{\mathrm{B}_{i_{1}}, \ldots, \mathrm{B}_{i_{k}}\right\}$, i.e., participants not in $\left\{\mathrm{B}_{i_{1}}, \ldots, \mathrm{B}_{i_{k}}\right\}$ are removed.

We write $s \backslash \mathrm{B}^{\prime}$ to indicate state projection onto $\mathrm{B}^{\prime}$, and define $s\left\lceil\mathrm{~B}^{\prime} \triangleq s\left\lceil\left\{\mathrm{~B}_{i_{1}}, \ldots, \mathrm{B}_{i_{k}}\right\}\right.\right.$, where $\mathrm{B}_{i_{1}}, \ldots, \mathrm{B}_{i_{k}}$ are the atomic components in $\mathrm{B}^{\prime}$. We say that $s \backslash \mathrm{B}^{\prime}$ is a state of $\mathrm{B}^{\prime}$. 
Definition 2.12 (Subsystem). Let $\left\{\mathrm{B}_{i_{1}}, \ldots, \mathrm{B}_{i_{k}}\right\} \subseteq\left\{\mathrm{B}_{1}, \ldots, \mathrm{B}_{n}\right\}$. Then the subsystem $\left(\mathrm{B}^{\prime}, Q_{0}^{\prime}\right)$ of $\left(\mathrm{B}, Q_{0}\right)$ based on $\left\{\mathrm{B}_{i_{1}}, \ldots, \mathrm{B}_{i_{k}}\right\}$ is as follows:

(1) $\mathrm{B}^{\prime}$ is the subcomponent of $\mathrm{B}$ based on $\left\{\mathrm{B}_{i_{1}}, \ldots, \mathrm{B}_{i_{k}}\right\}$

(2) $Q_{0}^{\prime}=Q_{0}\left\lceil\left\{\mathrm{~B}_{i_{1}}, \ldots, \mathrm{B}_{i_{k}}\right\}\right.$

Definition 2.13 (Execution projection). Let $\left(\mathrm{B}^{\prime}, Q_{0}^{\prime}\right)$, with $\mathrm{B}^{\prime}=\gamma^{\prime}\left(\mathrm{B}_{i_{1}}, \ldots, \mathrm{B}_{i_{k}}\right)$ be the subsystem of $\left(\mathrm{B}, Q_{0}\right)$ based on $\left\{\mathrm{B}_{i_{1}}, \ldots, \mathrm{B}_{i_{k}}\right\}$. Let $P^{\prime}=P_{i_{1}} \cup \cdots \cup P_{i_{k}}$, i.e., $P^{\prime}$ is the set of ports of $\left(\mathrm{B}^{\prime}, Q_{0}^{\prime}\right)$. Let $\rho=s_{0} \mathrm{a}_{1} s_{1} \ldots s_{j-1} \mathrm{a}_{j} s_{j} \ldots$ be an execution of $\left(\mathrm{B}, Q_{0}\right)$. Then, $\rho\left\lceil\left(\mathrm{B}^{\prime}, Q_{0}^{\prime}\right)\right.$, the projection of $\rho$ onto $\left(\mathrm{B}^{\prime}, Q_{0}^{\prime}\right)$, is the sequence resulting from:

(1) replacing each $s_{j}$ by $s_{j}\left\{\left\{\mathrm{~B}_{i_{1}}, \ldots, \mathrm{B}_{i_{k}}\right\}\right.$, i.e., replacing each state by its projection onto $\left\{\mathrm{B}_{i_{1}}, \ldots, \mathrm{B}_{i_{k}}\right\}$, then

(2) removing all $\mathrm{a}_{j} s_{j}$ where $\mathrm{a}_{j} \cap P^{\prime}=\emptyset$, then

(3) replacing each $a_{j}$ by $a_{j} \cap P^{\prime}$, i.e., replacing each interaction by its projection onto the port set $P^{\prime}$.

Proposition 2.14 (Execution projection). Let $\left(\mathrm{B}^{\prime}, Q_{0}^{\prime}\right)$, with $\mathrm{B}^{\prime}=\gamma^{\prime}\left(\mathrm{B}_{i_{1}}, \ldots, \mathrm{B}_{i_{k}}\right)$ be the subsystem of $\left(\mathrm{B}, Q_{0}\right)$ based on $\left\{\mathrm{B}_{i_{1}}, \ldots, \mathrm{B}_{i_{k}}\right\}$. Let $P^{\prime}=P_{i_{1}} \cup \cdots \cup P_{i_{k}}$, i.e., the union of the ports of $\left\{\mathrm{B}_{i_{1}}, \ldots, \mathrm{B}_{i_{k}}\right\}$. Let $\rho=s_{0} \mathrm{a}_{1} s_{1} \ldots s_{j-1} \mathrm{a}_{j} s_{j} \ldots$ be an execution of $\left(\mathrm{B}, Q_{0}\right)$. Then, $\rho \uparrow\left(\mathrm{B}^{\prime}, Q_{0}^{\prime}\right)$ is an execution of $\left(\mathrm{B}^{\prime}, Q_{0}^{\prime}\right)$.

Proof. By Definitions 2.10, 2.12, and 2.13, we have $\rho\left\lceil\left(\mathrm{B}^{\prime}, Q_{0}^{\prime}\right)=s_{0}^{\prime} \mathrm{b}_{1} s_{1}^{\prime} \mathrm{b}_{2} s_{2}^{\prime} \ldots\right.$ for some $s_{0}^{\prime}, \mathrm{b}_{1} s_{1}^{\prime} \mathrm{b}_{2} s_{2}^{\prime} \ldots$, where $s_{j}^{\prime} \in Q^{\prime}=Q\left\lceil\left\{\mathrm{~B}_{i_{1}}, \ldots, \mathrm{B}_{i_{k}}\right\}\right.$ for $j \geq 0$. Also by Definitions 2.10, 2.12, and 2.13, we have $s_{0}^{\prime} \in Q_{0}^{\prime}=Q_{0}\left\lceil\left\{\mathrm{~B}_{i_{1}}, \ldots, \mathrm{B}_{i_{k}}\right\}\right.$, since $s_{0}^{\prime}=s_{0}\left\lceil\mathrm{~B}^{\prime}\right.$, and $s_{0} \in Q_{0}$, by Definition 2.8.

Consider an arbitrary step $\left(s_{j-1}^{\prime}, \mathrm{b}_{j}, s_{j}^{\prime}\right)$ of $\rho\left\lceil\left(\mathrm{B}^{\prime}, Q_{0}^{\prime}\right)\right.$. Since $\mathrm{b}_{j} s_{j}^{\prime}$ was not removed in Clause 2 of Definition 2.13, we have

(1) $s_{j}^{\prime}=s_{\ell} \uparrow\left\{\mathrm{B}_{i_{1}}, \ldots, \mathrm{B}_{i_{k}}\right\}$ for some $\ell>0$ and such that $\mathrm{a}_{\ell} \cap P^{\prime} \neq \emptyset$

(2) $\mathrm{b}_{j}=\mathrm{a}_{\ell} \cap P^{\prime}$

(3) $s_{j-1}^{\prime}=s_{m}\left\lceil\left\{\mathrm{~B}_{i_{1}}, \ldots, \mathrm{B}_{i_{k}}\right\}\right.$ for the smallest $m$ such that

$m<\ell$ and $\forall m^{\prime}: m+1 \leq m^{\prime}<\ell: \mathrm{a}_{m^{\prime}} \cap P^{\prime}=\emptyset$

From (3) we have $\forall m^{\prime}: m+1 \leq m^{\prime}<\ell: \mathrm{a}_{m^{\prime}} \cap P^{\prime}=\emptyset$. So by Definitions 2.3 and 2.13, we have $s_{m}\left\lceil\left\{\mathrm{~B}_{i_{1}}, \ldots, \mathrm{B}_{i_{k}}\right\}=s_{\ell-1}\left\lceil\left\{\mathrm{~B}_{i_{1}}, \ldots, \mathrm{B}_{i_{k}}\right\}\right.\right.$. From (3) we have $s_{j-1}^{\prime}=s_{m}\left\lceil\left\{\mathrm{~B}_{i_{1}}, \ldots, \mathrm{B}_{i_{k}}\right\}\right.$. Hence $s_{j-1}^{\prime}=s_{\ell-1}\left\lceil\left\{\mathrm{~B}_{i_{1}}, \ldots, \mathrm{B}_{i_{k}}\right\}\right.$.

From $s_{\ell-1} \stackrel{\mathrm{a}_{\ell}}{\rightarrow} s_{\ell}, \mathrm{a}_{\ell} \cap P^{\prime} \neq \emptyset$, and Definition 2.3, we have $s_{\ell-1}\left\lceil\left\{\mathrm{~B}_{i_{1}}, \ldots, \mathrm{B}_{i_{k}}\right\} \stackrel{\mathrm{a}_{\ell} \cap P^{\prime}}{\rightarrow} s_{\ell}\left\lceil\left\{\mathrm{B}_{i_{1}}, \ldots, \mathrm{B}_{i_{k}}\right\}\right.\right.$. $s_{j-1}^{\prime}=s_{\ell-1}\left\lceil\left\{\mathrm{~B}_{i_{1}}, \ldots, \mathrm{B}_{i_{k}}\right\}\right.$ was established above. $s_{j}^{\prime}=s_{\ell}\left\lceil\left\{\mathrm{B}_{i_{1}}, \ldots, \mathrm{B}_{i_{k}}\right\}\right.$ is from $(1) . \mathrm{b}_{j}=\mathrm{a}_{\ell} \cap P^{\prime}$ is from (2). Hence we obtain $s_{j-1}^{\prime} \stackrel{\mathrm{b}_{j}}{\rightarrow} s_{j}^{\prime}$, i.e., that $s_{j-1}^{\prime}, \mathrm{b}_{j} s_{j}^{\prime}$ is a step of $\left(\mathrm{B}^{\prime}, Q_{0}^{\prime}\right)$.

Since $\left(s_{j-1}^{\prime}, \mathrm{b}_{j}, s_{j}^{\prime}\right)$ was arbitrarily chosen, we conclude that every step of $\rho \uparrow\left(\mathrm{B}^{\prime}, Q_{0}^{\prime}\right)$ is a step of $\left(\mathrm{B}^{\prime}, Q_{0}^{\prime}\right)$. This establishes Clause (2) of Definition 2.8. The first state of $\rho\left\lceil\left(\mathrm{B}^{\prime}, Q_{0}^{\prime}\right)\right.$ is $s_{0}^{\prime}$, and $s_{0}^{\prime} \in Q_{0}^{\prime}$ was shown above, so we establish Clause (1) of Definition 2.8.

Since both clauses of Definition 2.8 are satisfied, we conclude that $\rho\left\lceil\left(\mathrm{B}^{\prime}, Q_{0}^{\prime}\right)\right.$ is an execution of $\left(\mathrm{B}^{\prime}, Q_{0}^{\prime}\right)$.

Corollary 2.15. Let $\left(\mathrm{B}^{\prime}, Q_{0}^{\prime}\right)$ be a subsystem of $\left(\mathrm{B}, Q_{0}\right)$, and let $P^{\prime}$ be the port set of $\left(\mathrm{B}^{\prime}, Q_{0}^{\prime}\right)$. Let $s$ be a reachable state of $\left(\mathrm{B}, Q_{0}\right)$. Then $s \backslash \mathrm{B}^{\prime}$ is a reachable state of $\left(\mathrm{B}^{\prime}, Q_{0}^{\prime}\right)$. Let $s \stackrel{\mathrm{a}}{\rightarrow} t$ be a reachable transition of $\left(\mathrm{B}, Q_{0}\right)$, and let $\mathrm{a} \cap P^{\prime}$ be an interaction of $\left(\mathrm{B}^{\prime}, Q_{0}^{\prime}\right)$. Then $s\left\lceil\mathrm{~B}^{\prime} \stackrel{\mathrm{a} \cap P^{\prime}}{\rightarrow} t\left\lceil\mathrm{~B}^{\prime}\right.\right.$ is a reachable transition of $\left(\mathrm{B}^{\prime}, Q_{0}^{\prime}\right)$.

Proof. Immediate corollary of Proposition 2.14 . 
Example 2.16 (Execution projection). In the dining philosophers example of Figure 1, let the (single) initial state be $\left(h_{0}, h_{1}, h_{2}, h_{3}, f_{0}, f_{1}, f_{2}, f_{3}\right)$, i.e., all philosophers are hungry and all forks are free. Also, Grab $b_{0}=\left\{\right.$ get $_{0}$, use $_{0}^{\ell}$, use $\left._{1}^{r}\right\}$ (resp. Grab $2=\left\{\right.$ get $_{2}$, use $_{2}^{\ell}$, use $\left.\left._{3}^{r}\right\}\right)$ is the interaction in which $P h_{0}$ (resp. $P h_{2}$ ) picks up both forks, and $R e l_{0}=\left\{p u t_{0}\right.$, free $_{0}^{\ell}$, free $\left._{1}^{r}\right\}$ is the interaction in which $P h_{0}$ releases both forks. Consider the following execution: $\rho=\left(h_{0}, h_{1}, h_{2}, h_{3}, f_{0}, f_{1}, f_{2}, f_{3}\right) \quad\left\{\right.$ get $_{0}$, use $_{0}^{\ell}$, use $\left._{1}^{r}\right\} \quad\left(e_{0}, h_{1}, h_{2}, h_{3}, u_{0}^{\ell}, u_{1}^{r}, f_{2}, f_{3}\right) \quad$ get $_{2}$, use $_{2}^{\ell}$, use $\left._{3}^{r}\right\}$ $\left(e_{0}, h_{1}, e_{2}, h_{3}, u_{0}^{\ell}, u_{1}^{r}, u_{2}^{\ell}, u_{3}^{r}\right)\left\{p u t_{0}\right.$, free $_{0}^{\ell}$, free $\left._{1}^{r}\right\} \quad\left(h_{0}, h_{1}, e_{2}, h_{3}, f_{0}, f_{1}, u_{2}^{\ell}, u_{3}^{r}\right) \cdots$. The projection of this execution on the subsystem defined by subcomponent $\left\{P h_{0}, P h_{1}, F_{0}\right\}$, is equal to: $\rho\left\lceil\left\{P h_{0}, P h_{1}, F_{0}\right\}, Q_{0}^{\prime}\right)=\left(h_{0}, h_{1}, f_{0}\right)\left\{\right.$ get $\left._{0}, u s e_{0}^{\ell}\right\}\left(e_{0}, h_{1}, u_{0}^{\ell}\right)\left\{\right.$ put $_{0}$, free $\left._{0}^{\ell}\right\}\left(h_{0}, h_{1}, f_{0}\right) \cdots$. In particular, we project the states and interactions with respect to the subcomponent. Notice that interaction $\mathrm{Grab}_{2}$ disappears as its ports do not belong to the subcomponent. Clearly, $\rho \uparrow\left(\left\{P h_{0}, P h_{1}, F_{0}\right\}, Q_{0}^{\prime}\right)$ is an execution of $\left(\left\{P h_{0}, P h_{1}, F_{0}\right\}, Q_{0}^{\prime}\right)$.

\section{CHARACTERIZING DEADLOCK-FREEDOM}

Definition 3.1 (Global deadlock-freedom). A BIP-system $\left(\mathrm{B}, Q_{0}\right)$ is free of global deadlock iff in every reachable state $s$ of $\left(\mathrm{B}, Q_{0}\right)$, some interaction a is enabled. Formally, $\forall s \in \operatorname{rstates}\left(\mathrm{B}, Q_{0}\right), \exists \mathrm{a}: s \stackrel{\mathrm{a}}{\longrightarrow} \mathrm{B}$.

Definition 3.2 (Local deadlock-freedom). A BIP-system (B, $\left.Q_{0}\right)$ is free of local deadlock iff for every subsystem $\left(\mathrm{B}^{\prime}, Q_{0}^{\prime}\right)$ of $\left(\mathrm{B}, Q_{0}\right)$, and every reachable state $s$ of $\left(\mathrm{B}, Q_{0}\right),\left(\mathrm{B}^{\prime}, Q_{0}^{\prime}\right)$ has some interaction enabled in state $s \backslash \mathrm{B}^{\prime}$. Formally:

for every subsystem $\left(\mathrm{B}^{\prime}, Q_{0}^{\prime}\right)$ of $\left(\mathrm{B}, Q_{0}\right)$ :

$$
\forall s \in \operatorname{rstates}\left(\mathrm{B}, Q_{0}\right), \exists \mathrm{a}: s\left\lceil\mathrm{~B}^{\prime} \stackrel{\mathrm{a} \cap P^{\prime}}{\longrightarrow} \mathrm{B}^{\prime}\right.
$$

where $P^{\prime}$ is the set of ports of $\mathrm{B}^{\prime}$.

Note that every reachable state $s^{\prime}$ of subsystem $\mathrm{B}^{\prime}$ (within the context of the overall system B) is a projection of a reachable state $s$ of $\mathrm{B}$, i.e., $s^{\prime}=s\left\lceil\mathrm{~B}^{\prime}\right.$. Our definition requires that, in every reachable state $s^{\prime}, \mathrm{B}^{\prime}$ is not prevented from executing some interaction a due to blocking relationships within $\mathrm{B}^{\prime}$, which would constitute a local deadlock. We thus require that $\mathrm{B}^{\prime}$, considered in isolation from its containing system $\mathrm{B}$, must enable some interaction a. Within $\mathrm{B}$ overall, it is permissible for a to be disabled because some $\mathrm{B}_{i} \in$ components(a) does not enable a, provided that $\mathrm{B}_{i}$ is not a component of $\mathrm{B}^{\prime}$. We now make these ideas precise.

\subsection{Wait-for graphs}

The wait-for graph for a state $s$ is a directed bipartite and-or graph which contains as nodes the atomic components $\mathrm{B}_{1}, \ldots, \mathrm{B}_{n}$, and all the interactions $\gamma$. Edges in the wait-for-graph are from a component $\mathrm{B}_{i}$ to all the interactions that $\mathrm{B}_{i}$ enables (in $s$ ), and from an interaction a to all the components that participate in a and which do not enable it (in $s$ ).

Definition 3.3 (Wait-for graph $W_{\mathrm{B}}(s)$ ). Let $\mathrm{B}=\gamma\left(\mathrm{B}_{1}, \ldots, \mathrm{B}_{n}\right)$ be a BIP composite component, and let $s=\left\langle s_{1}, \ldots, s_{n}\right\rangle$ be an arbitrary state of $\mathrm{B}$. The wait-for graph $W_{\mathrm{B}}(s)$ of $s$ is a directed bipartite and-or graph, where

1. the nodes of $W_{\mathrm{B}}(s)$ are as follows:

(a) the and-nodes are the atomic components $\mathrm{B}_{i}, i \in\{1 . . n\}$,

(b) the or-nodes are the interactions $a \in \gamma$,

2. there is an edge in $W_{\mathrm{B}}(s)$ from $\mathrm{B}_{i}$ to every node a such that $\mathrm{B}_{i} \in \operatorname{components}(\mathrm{a})$ and $s_{i}\left(e n b_{\mathrm{a}}^{i}\right)=$ true, i.e., from $\mathrm{B}_{i}$ to every interaction which $\mathrm{B}_{i}$ enables in $s_{i}$,

3. there is an edge in $W_{\mathrm{B}}(s)$ from a to every $\mathrm{B}_{i}$ such that $\mathrm{B}_{i} \in$ components $(\mathrm{a})$ and $s_{i}\left(e n b_{\mathrm{a}}^{i}\right)=$ false, i.e., from a to every component $\mathrm{B}_{i}$ which participates in a but does not enable it, in state $s_{i}$. 
A component $\mathrm{B}_{i}$ is an and-node since all of its successor actions (or-nodes) must be disabled for $B_{i}$ to be incapable of executing. An interaction a is an or-node since it is disabled if any of its participant components do not enable it. An edge (path) in a wait-for graph is called a wait-for edge (wait-for path).

Definition 3.4 (Subgraph of a wait-for graph). $U$ is a subgraph of $W_{\mathrm{B}}(s)$ iff the nodes of $U$ are a subset of the nodes of $W_{\mathrm{B}}(s)$ and the edges of $U$ are the induced edges from $W_{\mathrm{B}}(s)$, i.e., if $u, v$ are nodes of $U$ and $u \rightarrow v$ is an edge of $W_{\mathrm{B}}(s)$, then $u \rightarrow v$ is an edge of $U$. Write $U \sqsubseteq W_{\mathrm{B}}(s)$ when $U$ is a subgraph of $W_{\mathrm{B}}(s)$, and extend the definition of $\sqsubseteq$ to subgraphs of $W_{\mathrm{B}}(s)$ in the obvious manner, so that $U \sqsubseteq V$ means that $U$ is a subgraph of $V$.

Write a $\rightarrow \mathrm{B}_{i}\left(\mathrm{~B}_{i} \rightarrow\right.$ a respectively) for a wait-for edge from a to $\mathrm{B}_{i}$ ( $\mathrm{B}_{i}$ to a respectively). We abuse notation by writing $v \in W_{\mathrm{B}}(s)$ to indicate that $v$ is a node of $W_{\mathrm{B}}(s)$, and $e \in W_{\mathrm{B}}(s)$ to indicate that $e$ (either $\mathrm{a} \rightarrow \mathrm{B}_{i}$ or $\mathrm{B}_{i} \rightarrow$ a) is an edge in $W_{\mathrm{B}}(s)$. Also $\mathrm{B}_{i} \rightarrow \mathrm{a} \rightarrow \mathrm{B}_{i}^{\prime} \in W_{\mathrm{B}}(s)$ for $\mathrm{B}_{i} \rightarrow \mathrm{a} \in W_{\mathrm{B}}(s) \wedge \mathrm{a} \rightarrow \mathrm{B}_{i}^{\prime} \in W_{\mathrm{B}}(s)$, i.e., for a wait-for path of length 2 , and similarly for longer wait-for paths. Likewise use $v \in U, e \in U$, where $U$ is a subgraph of $W_{\mathrm{B}}(s)$.

Consider the dining philosophers system given in Figure 1. Figure 2(a) shows its wait-for graph in its sole initial state. Figure 2(b) shows the wait-for graph after execution of $G r a b_{0}$. In all figures of wait-for graphs, we show components in red, interactions in blue, edges from components to interactions as solid, and edges from interactions to components as dashed.

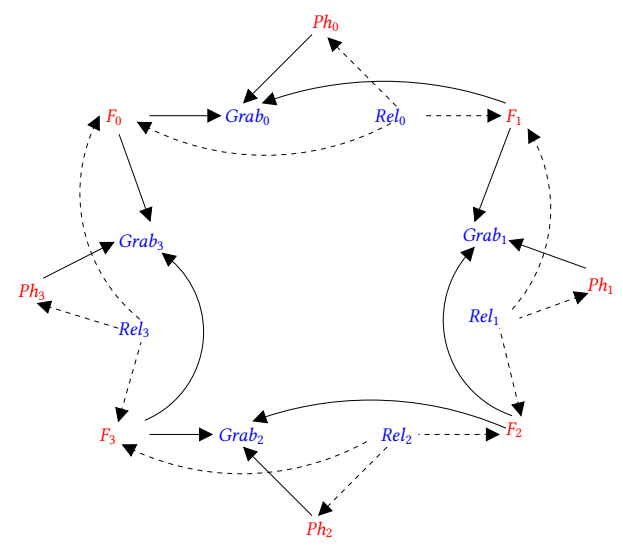

(a) Wait-for-graph in initial state.

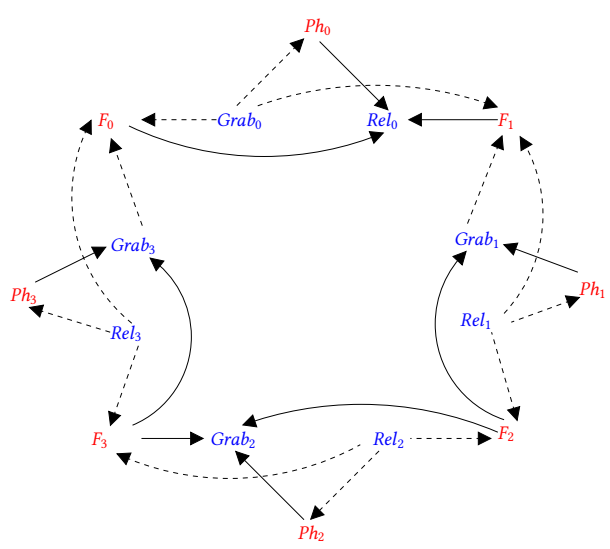

(b) Wait-for-graph after execution of $G r a b_{0}$.

Fig. 2. Example wait-for-graphs for dining philosophers system of Figure 1.

A key principle of the dynamics of the change of wait-for graphs is that wait-for edges not involving some interaction a and its participants $\mathrm{B}_{i} \in$ components $(\mathrm{a})$ are unaffected by the execution of a. Say that edge $e$ in a wait-for graph is $\mathrm{B}_{i}$-incident iff $\mathrm{B}_{i}$ is one of the endpoints of $e$.

Proposition 3.5 (WAIT-For Edge Preservation). Let $s \stackrel{\text { a }}{\rightarrow} t$ be a transition of composite component $\mathrm{B}=\gamma\left(\mathrm{B}_{1}, \ldots, \mathrm{B}_{n}\right)$, and let e be a wait-for edge in $W_{\mathrm{B}}(s)$ that is not $\mathrm{B}_{i}$-incident, for every $\mathrm{B}_{i} \in$ components $(\mathrm{a})$. Then $e \in W_{\mathrm{B}}(s)$ iffe $e \in W_{\mathrm{B}}(t)$.

Proof. Fix $e$ to be an arbitrary wait-for-edge that is not $\mathrm{B}_{i}$-incident. $e$ is either $\mathrm{B}_{j} \rightarrow \mathrm{b}$ or $\mathrm{b} \rightarrow \mathrm{B}_{j}$, for some component $\mathrm{B}_{j}$ of $\mathrm{B}$ that is not in components $(\mathrm{a})$, and an interaction $\mathrm{b}$ (different from $\mathrm{a}$ ) that 
$\mathrm{B}_{j}$ participates in. Now $s\left\lceil\mathrm{~B}_{j}=t\left\lceil\mathrm{~B}_{j}\right.\right.$, since $s \stackrel{\mathrm{a}}{\rightarrow} t$ and $\mathrm{B}_{j} \notin$ components $(\mathrm{a})$. Hence $s\left(e n b_{\mathrm{b}}^{j}\right)=t\left(e n b_{\mathrm{b}}^{j}\right)$. It follows from Definition 3.3 that $e \in W_{\mathrm{B}}(s)$ iff $e \in W_{\mathrm{B}}(t)$.

\subsection{Supercycles and deadlock-freedom}

We characterize a deadlock as the existence in the wait-for graph of a graph-theoretic construct that we call a supercycle.

Definition 3.6 (Supercycle). Let $\mathrm{B}=\gamma\left(\mathrm{B}_{1}, \ldots, \mathrm{B}_{n}\right)$ be a composite component and $s$ be a state of B. A subgraph $S C$ of $W_{\mathrm{B}}(s)$ is a supercycle in $W_{\mathrm{B}}(s)$ if and only if all of the following hold:

1. $S C$ is nonempty, i.e., contains at least one node,

2. if $\mathrm{B}_{i}$ is a node in $S C$, then for all interactions a such that there is an edge in $W_{\mathrm{B}}(s)$ from $\mathrm{B}_{i}$ to a:

(a) a is a node in $S C$, and

(b) there is an edge in $S C$ from $\mathrm{B}_{i}$ to a,

that is, $\mathrm{B}_{i} \rightarrow \mathrm{a} \in W_{\mathrm{B}}(s)$ implies $\mathrm{B}_{i} \rightarrow \mathrm{a} \in S C$,

3. if a is a node in $S C$, then there exists a $\mathrm{B}_{j}$ such that:

(a) $\mathrm{B}_{j}$ is a node in $S C$, and

(b) there is an edge from a to $\mathrm{B}_{j}$ in $W_{\mathrm{B}}(s)$, and

(c) there is an edge from a to $\mathrm{B}_{j}$ in $S C$,

that is, $\mathrm{a} \in S C$ implies $\exists \mathrm{B}_{j}: \mathrm{a} \rightarrow \mathrm{B}_{j} \in W_{\mathrm{B}}(s) \wedge \mathrm{a} \rightarrow \mathrm{B}_{j} \in S C$. $S C$.

Intuitively, $S C$ is a supercycle iff every node is $S C$ is blocked from executing by other nodes in

Definition 3.7 (Supercycle-free). $W_{\mathrm{B}}(s)$ is supercycle-free iff there does not exist a supercycle $S C$ in $W_{\mathrm{B}}(s)$. In this case, say that state $s$ is supercycle-free.

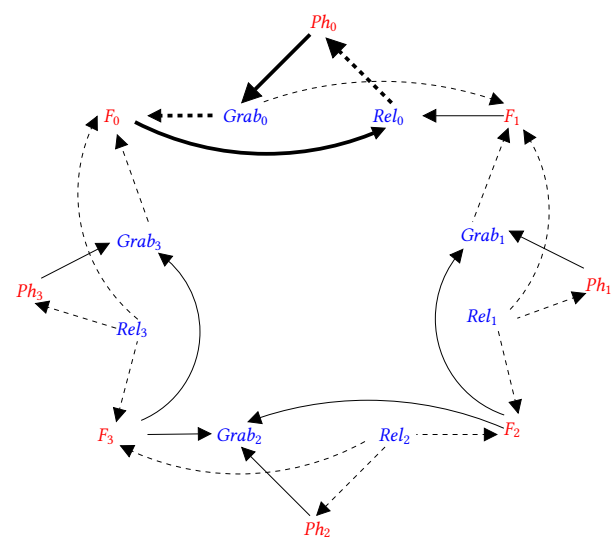

Fig. 3. Example supercycle for dining philosophers system of Figure 1.

Figure 3 shows an example supercycle (with edges in bold) for the dining philosophers system of Figure 1. $P h_{0}$ waits for (enables) a single interaction, $G r a b_{0}$. Grab $b_{0}$ waits for (is disabled by) fork $F_{0}$, which waits for interaction $R e l_{0}$. $R e l_{0}$ in turn waits for $P h_{0}$. However, this supercycle occurs when $P h_{0}$ is in state $h_{0}$ and $F_{0}$ is in state $u_{0}^{\ell}$. This state is not reachable from the initial state.

Figure 4 shows an example of a supercycle that is not a simple cycle. The "essential" part of the supercycle, consisting of components $\mathrm{B}_{1}, \mathrm{~B}_{2}, \mathrm{~B}_{3}$, and their interactions $\mathrm{a}, \mathrm{b}, \mathrm{c}, \mathrm{d}$, is in bold. The 
supercycle can be extended to contain $B_{4}$, but neither $B_{5}$ nor $B_{6}: B_{6}$ is enabled, and $B_{5}$ is ready to execute the interaction $h$, which waits only for $B_{6}$. Figure 5 shows that deleting the wait-for edge

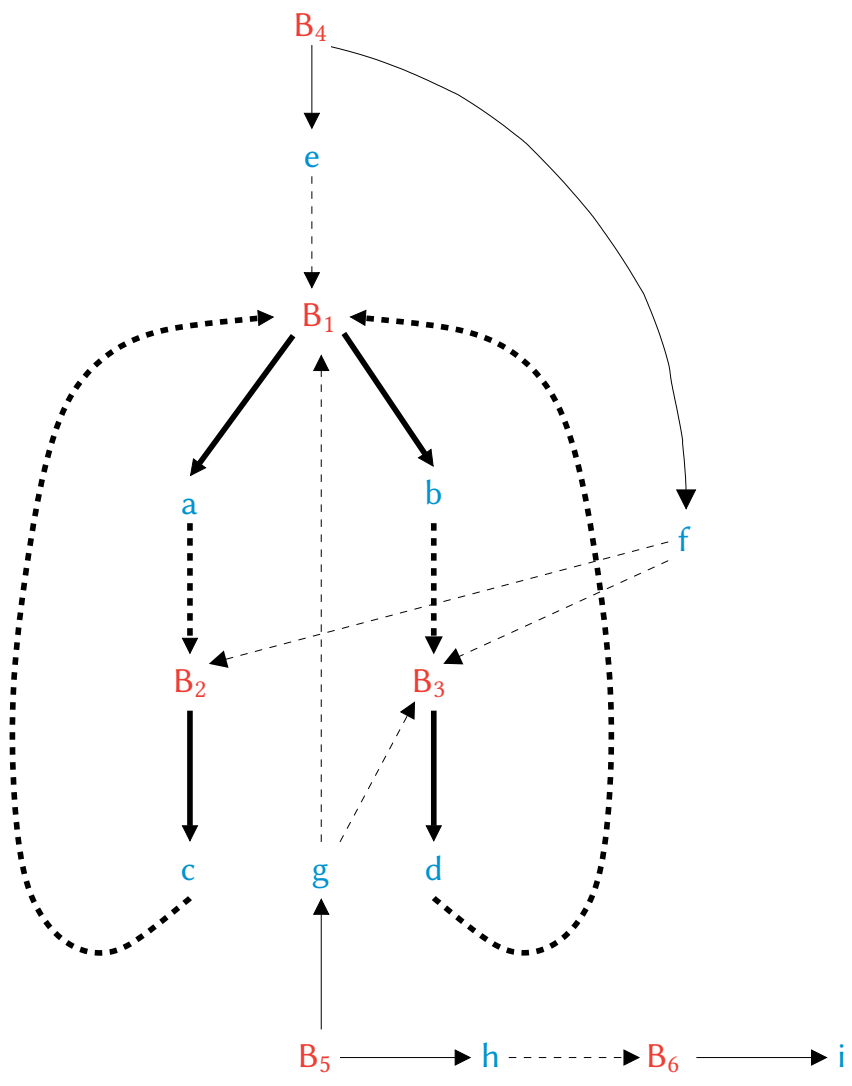

Fig. 4. Example supercycle that is not a simple cycle.

from $d$ to $B_{1}$ in Figure 4 results in an example where there is a cycle of wait-for edges, without there being a supercycle. This shows that a cycle does not necessarily imply a supercycle, and hence deadlock.

The existence of a supercycle is sufficient and necessary for the occurrence of a deadlock, and so checking for supercycles gives a sound and complete check for deadlocks. Proposition 3.8 states that the existence of a supercycle implies a local deadlock: all components in the supercycle are blocked forever.

Proposition 3.8. Let s be a state of $\mathrm{B}$. If $S C \sqsubseteq W_{\mathrm{B}}(s)$ is a supercycle, then all atomic components $\mathrm{B}_{i}$ in $\mathrm{SC}$ cannot execute a transition in any state $u$ reachable from $s$, including $s$ itself.

Proof. Let $\mathrm{B}_{i}$ be an arbitrary component in $S C$. By Definition 3.6, every interaction that $\mathrm{B}_{i}$ enables has a wait-for edge to some other component $\mathrm{B}_{j}$ in $S C$ and so cannot be executed in state $s$. Hence in any transition from $s$ to another global state $t$, all of the components $\mathrm{B}_{i}$ in $S C$ remain in the same local state. Hence $S C \sqsubseteq W_{\mathrm{B}}(t)$, i.e., the same supercycle $S C$ remains in global state $t$. Repeating this argument from state $t$ and onwards leads us to conclude that $S C \sqsubseteq W_{\mathrm{B}}(u)$ for any state $u$ reachable from $s$. 


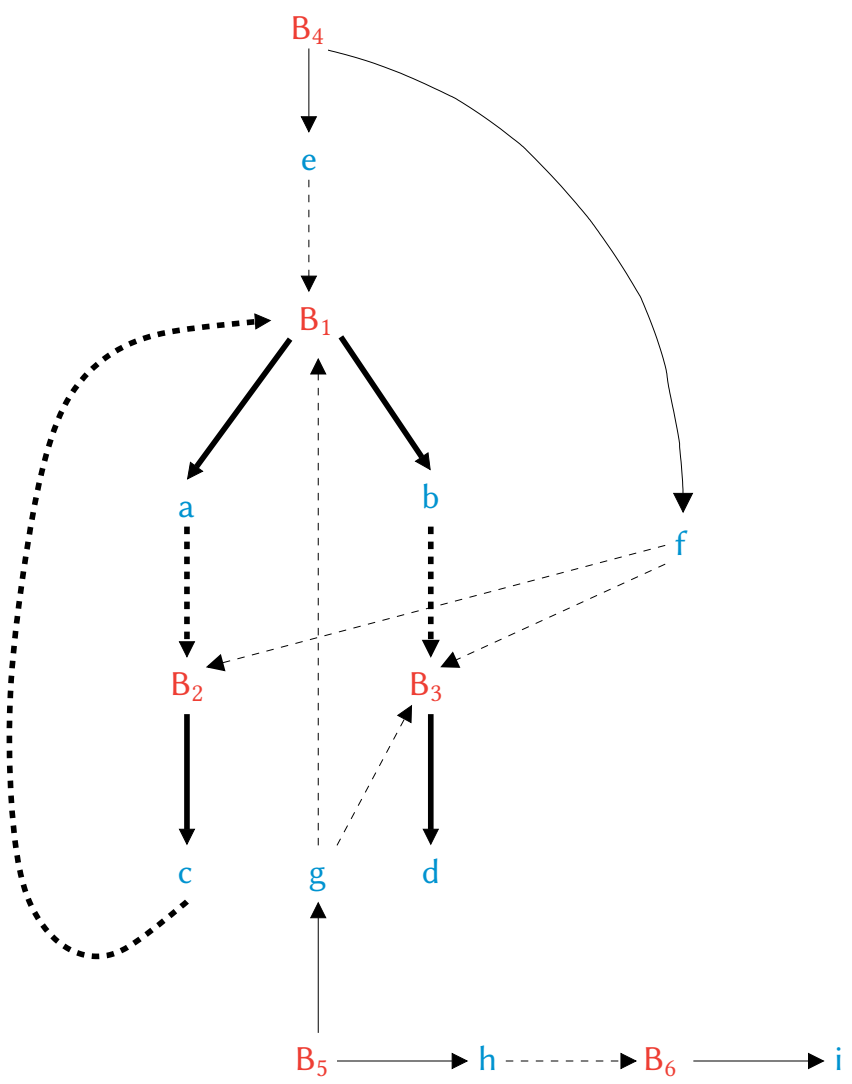

Fig. 5. Example where a wait-for cycle does not imply deadlock.

Proposition 3.9 states that the existence of a supercycle is necessary for a local deadlock to occur: if a set of components, considered in isolation, are blocked, then there exists a supercycle consisting of exactly those components, together with the interactions that each component enables.

Proposition 3.9. Let $\mathrm{B}^{\prime}$ be a subcomponent of $\mathrm{B}$, and let $s$ be an arbitrary state of $\mathrm{B}$ such that $\mathrm{B}^{\prime}$, when considered in isolation, has no enabled interaction in state $s \backslash \mathrm{B}^{\prime}$. Then, $W_{\mathrm{B}}(s)$ contains a supercycle.

Proof. Let $\mathrm{B}_{i}$ be an arbitrary atomic component in $\mathrm{B}^{\prime}$, and let a be any interaction that $\mathrm{B}_{i}$ enables. Since $\mathrm{B}^{\prime}$ has no enabled interaction, it follows that a is not enabled in $\mathrm{B}^{\prime}$, and therefore has a wait-for edge to some atomic component $\mathrm{B}_{j}$ in $\mathrm{B}^{\prime}$. Hence let $S C$ be the subgraph of $W_{\mathrm{B}}(s)$ induced by:

(1) the atomic components of $\mathrm{B}^{\prime}$,

(2) the interactions a that each atomic component $\mathrm{B}_{i}$ enables, and the edges $\mathrm{B}_{i} \rightarrow \mathrm{a}$, and

(3) the edges $\mathrm{a} \rightarrow \mathrm{B}_{j}$ from each interaction a to some atomic component $\mathrm{B}_{j}$ in $\mathrm{B}^{\prime}$, where $\mathrm{B}_{j}$ does not enable a.

$S C$ satisfies Definition 3.6 and so is a supercycle. 
We consider subcomponent $\mathrm{B}^{\prime}$ in isolation to avoid other phenomena that prevent interactions from executing, e.g., conspiracies [9]. Now the contrapositive of Proposition 3.9 is that absence of supercycles in $W_{\mathrm{B}}(s)$ means there is no locally deadlocked subsystem.

COROLLARY 3.10 (SUPERCYCLE-FREE IMPLIES FREE OF LOCAL DEADLOCK). If, for every reachable state $s$ of $\left(\mathrm{B}, Q_{0}\right), W_{\mathrm{B}}(s)$ is supercycle-free, then $\left(\mathrm{B}, Q_{0}\right)$ is free of local deadlock.

Proof. Suppose that $\left(\mathrm{B}, Q_{0}\right)$ is not free of local deadlock. Then there exists a subsystem $\left(\mathrm{B}^{\prime}, Q_{0}^{\prime}\right)$ of $\left(\mathrm{B}, Q_{0}\right)$, and a reachable state $s$ of $\left(\mathrm{B}^{\prime}, Q_{0}^{\prime}\right)$, such that $\mathrm{B}^{\prime}$ enables no interaction in state $s\left\lceil\mathrm{~B}^{\prime}\right.$. By Proposition 3.9, $W_{\mathrm{B}}(s)$ contains a supercycle.

\subsection{Subsystems and Supercycles}

In the sequel, we say "deadlock-free" to mean "free of local deadlock". We wish to check whether supercycles can be formed or not. In principle, we could check directly whether $W_{\mathrm{B}}(s)$ contains a supercycle, for each reachable state $s$. However, this approach is subject to state-explosion, and so is usually unlikely to be viable in practice. Instead, we formulate global conditions for supercycle-freedom, and then "project" these conditions onto small subsystems, to obtain local versions of these conditions that are (1) efficiently checkable, and (2) imply the global versions. To formulate the global conditions, we characterize the static (structural) and dynamic (formation) properties of supercycles, in Sections 4 and 5, respectively. To define the projection of the global deadlock-freedom conditions onto small subsystems, we present the notion of local supercycle in Section 6. For each interaction a in the BIP-system $\left(\mathrm{B}, Q_{0}\right)$, the local check computes a subsystem which includes a and also other components/interactions at a given "distance" from a. It then checks whether any of the subsystem components is involved in a local supercycle.

Figure 6 illustrates the wait-for graph (in the initial state) of the dining philosopher subsystem corresponding to the $G r a b_{0}$ interaction with a distance of 1, i.e., its components and their interactions. The subsystem includes the $G r a b_{0}$ interaction, the components $P h_{0}, F_{0}$, and $F_{1}$ that participate in $\mathrm{Grab}_{0}$, and the interactions $\operatorname{Rel}_{0}, \mathrm{Grab}_{1}, \mathrm{Rel}_{1}, \mathrm{Grab}_{3}$, and $\mathrm{Rel}_{3}$ which have at least one of these components as participants. We notice that no component in Figure 6 is involved in a supercycle. The interactions $\mathrm{Grab}_{1}, \mathrm{Rel}_{1}, \mathrm{Grab}_{3}$, and $\mathrm{Rel}_{3}$ (underlined in the figure) are "border interactions", since they have participating components that are outside the subsystem. The enablement of border interactions cannot be determined from the subsystem in isolation. Hence, to ensure soundness of our supercycle-freedom check, we must assume pessimistically that the border interactions are not enabled. This pessimism may yield a false negative, thereby causing our check to be incomplete. The further the distance is increased, the "more complete" the local check becomes, as the local states of the larger subsystem give a better over-approximation to the global states of the entire system.

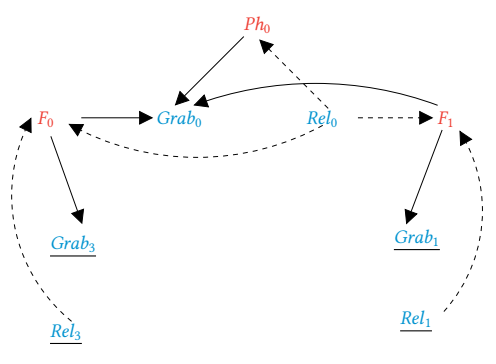

Fig. 6. Wait-for graph in the initial state of the dining philosophers subsystem for $G r a b_{0}$ and distance 1. 


\subsection{Abstract supercycle freedom conditions}

Since we will present several conditions for supercycle-freedom, we now present an abstract definition of the essential properties that all such conditions must have. The key idea is that execution of an interaction a does not create a supercycle, and so any condition which implies this for $a$ is sufficient. If a different condition implies the same for another interaction aa, this presents no problem w.r.t. establishing deadlock-freedom. Hence, it is sufficient to have one such condition for each interaction in $\left(\mathrm{B}, Q_{0}\right)$. Since each condition restricts the behavior of interaction execution, we call it a "behavioral restriction condition".

Definition 3.11 (Behavioral restriction condition). A behavioral restriction condition $\mathcal{B C}$ is a predicate $\mathcal{B C}:\left(\mathrm{B}, Q_{0}, \mathrm{a}\right) \rightarrow$ true, false $\}$.

$\mathcal{B C}$ is a predicate on the effects of a particular interaction a within a given system $\left(\mathrm{B}, Q_{0}\right)$.

Definition 3.12 (Supercycle-freedom preserving). A behavioral restriction condition $\mathcal{B C}$ is supercycle-freedom preserving iff, for every BIP-system (B, $\left.Q_{0}\right)$ and interaction a $\in \gamma$ of $\left(\mathrm{B}, Q_{0}\right)$ : if $\mathcal{B C}\left(\mathrm{B}, Q_{0}, \mathrm{a}\right)=$ true, then

for every reachable transition $t \stackrel{\text { a }}{\rightarrow} s$ of $\left(\mathrm{B}, Q_{0}\right)$

if $t$ is supercycle-free, then $s$ is supercycle-free.

THEOREM 3.13 (DEADLOCK-FREEDOM VIA SUPERCYCLE-FREEDOM PRESERVING RESTRICTION). Assume that

(1) for all $s_{0} \in Q_{0}, W_{\mathrm{B}}\left(s_{0}\right)$ is supercycle-free, and

(2) there exists a supercycle-freedom preserving restriction $\mathcal{B C}$ such that, for all a $\in \gamma$ : $\mathcal{B C}\left(\mathrm{B}, Q_{0}, \mathrm{a}\right)=$ true.

Then for every reachable state $u$ of $\left(\mathrm{B}, Q_{0}\right): W_{\mathrm{B}}(u)$ is supercycle-free.

Proof. Let $u$ be an arbitrary reachable state. The proof is by induction on the length of the finite execution $\alpha$ that ends in $u$. Assumption 1 provides the base case, for $\alpha$ having length 0 , and so $u \in Q_{0}$. For the induction step, we establish: for every reachable transition $t \stackrel{\text { a }}{\rightarrow} s, W_{\mathrm{B}}(t)$ is supercycle-free implies that $W_{\mathrm{B}}(s)$ is supercycle-free. This is immediate from Assumption 2, and Definition 3.12.

Since the above proof does not make any use of the requirement that there is a single restriction $\mathcal{B C}$ for all interactions, we immediately have:

COROLLARY 3.14 (DEADLOCK-FREEDOM VIA SEVERAL SUPERCYCLE-FREEDOM PRESERVING RESTRICTIONS). Assume that

(1) for all $s_{0} \in Q_{0}, W_{\mathrm{B}}\left(s_{0}\right)$ is supercycle-free, and

(2) for all a $\in \gamma$, there exists a supercycle-freedom preserving restriction $\mathcal{B C}$ such that $\mathcal{B C}\left(\mathrm{B}, Q_{0}, \mathrm{a}\right)=$ true.

Then for every reachable state $u$ of $\left(\mathrm{B}, Q_{0}\right): W_{\mathrm{B}}(u)$ is supercycle-free.

Proof. Similar to the proof of Theorem 3.13, except that, for the transition $t \stackrel{\text { a }}{\rightarrow} s$, use the supercycle-freedom preserving restriction $\mathcal{B C}$ corresponding to a.

\section{GLOBAL SUPERCYCLES}

Recall that $\left(\mathrm{B}, Q_{0}\right)$ is an arbitrary fixed BIP-system, (with $\mathrm{B}=\gamma\left(\mathrm{B}_{1}, \ldots, \mathrm{B}_{n}\right)$ ), which we use in all definitions, theorems etc. We characterize a supercycle as a post-fixpoint of a "blocking operator" $\mathcal{S}$ (defined below) over the complete Boolean lattice formed from the subgraphs of $W_{\mathrm{B}}(s)$, with $\sqsubseteq$ 
(Definition 3.4) as the ordering. Roughly, $\mathcal{S}$ maps a subset $X$ of the nodes of $W_{\mathrm{B}}(s)$ (i.e., some subset of the components and interactions in $\left.\left(\mathrm{B}, Q_{0}\right)\right)$ to a set of nodes $Y$ whose execution is blocked by $X$. An interaction a in $Y$ is blocked by $X$ if some participant of a is in $X$ and does not enable a. A component $\mathrm{B}_{i}$ in $Y$ is blocked by $X$ if every interaction that $\mathrm{B}_{i}$ enables is in $X$. In terms of $W_{\mathrm{B}}(s)$, a is blocked by $X$ if there is a wait-for edge from a to some node in $X$, and $\mathrm{B}_{i}$ is blocked by $X$ if every wait-for edge from $B_{i}$ is to a node in $X$.

Since $\mathcal{S}$ is monotone, its greatest fixpoint $S C$ exists. If $W_{\mathrm{B}}(s)$ is supercycle-free, then $S C$ is the empty wait-for graph $\emptyset$. Otherwise $S C$ is the largest supercycle in $W_{\mathrm{B}}(s)$. We define the dual $\mathcal{V}$ of $\mathcal{S}$, whose least fixpoints are the nodes that are not members of any supercycle, and we say that such nodes have a supercycle violation. Since $\mathcal{V}$ is monotone and continuous, and the underlying lattice is finite, its least fixpoint can be computed as usual by iterating $\mathcal{V}$, starting from $\emptyset$. This provides a method of computing the nodes with supercycle violations, which is the basis for our deadlock-freedom criterion.

\subsection{A fixpoint characterization of supercycles}

Definition 4.1 (Set of subgraphs). $\mathcal{P}\left(W_{\mathrm{B}}(s)\right)=\left\{X \mid X \sqsubseteq W_{\mathrm{B}}(s)\right\}$.

We include in $\mathcal{P}\left(W_{\mathrm{B}}(s)\right)$ the empty wait-for graph, which we denote by $\emptyset$. Let nodes $(\mathrm{B})=$ $\left\{\mathrm{B}_{1}, \ldots, \mathrm{B}_{n}\right\} \cup \gamma$, i.e., nodes $(\mathrm{B})$ is the set of components and interactions in $\mathrm{B}$, and let $\mathcal{P}($ nodes $(\mathrm{B}))$ be the powerset of nodes $(\mathrm{B})$. Then $\mathcal{P}\left(W_{\mathrm{B}}(s)\right)$ is isomorphic to $\mathcal{P}(\operatorname{nodes}(\mathrm{B}))$, where each $X \in \mathcal{P}\left(W_{\mathrm{B}}(s)\right)$ is mapped to the set of nodes that it contains.

Definition 4.2 (Wait-for lattice). Define the partially ordered set $\mathcal{L}_{\mathrm{B}}(s)=\left\langle\mathcal{P}\left(W_{\mathrm{B}}(s)\right.\right.$ ), ㄷ whose elements are all the subgraphs of $W_{\mathrm{B}}(s)$, and where $U \sqsubseteq V$ is as in Definition 3.4.

The following proposition follows immediately from the definitions; its proof is left to the reader.

Proposition 4.3. $\mathcal{L}_{\mathrm{B}}(s)=\left\langle\mathcal{P}\left(W_{\mathrm{B}}(s)\right)\right.$, $\left.\sqsubseteq\right\rangle$ is a finite complete Boolean lattice as follows:

- meet is given by graph intersection: $X \sqcap Y$ consists of the nodes that are present in both $X$ and $Y$, together with the edges induced by $W_{\mathrm{B}}(s)$, i.e., if $u \in X \sqcap Y, v \in X \sqcap Y$, and $u \rightarrow v \in W_{\mathrm{B}}(s)$, then $u \rightarrow v \in X \sqcap Y$.

- join is given by graph union: $X \sqcup Y$ consists of the nodes that are present in $X$, or in $Y$, or in both, together with the edges induced by $W_{\mathrm{B}}(s)$. Note that $\sqcup$ is not disjoint graph union: it is possible for $X$ and $Y$ to have nodes and edges in common. Note also that $X \sqcup Y$ may contain edges not present in either $X$ nor $Y$, since the edges are those induced by $W_{\mathrm{B}}(s)$.

- $W_{\mathrm{B}}(s)$ is the top element.

- the empty wait-for graph $\emptyset$ is the bottom element.

- the complement $\bar{X}$ of $X$ is obtained by taking all the nodes of $W_{\mathrm{B}}(s)$ that are not in $X$, together with the induced edges.

As noted, $\sqcup, \sqcap$ and complement are determined entirely by the sets of nodes in the relevant subgraphs. The resulting edges are always those that are induced by $W_{\mathrm{B}}(s)$. Let $\langle\mathcal{P}(\operatorname{nodes}(\mathrm{B})), \subseteq\rangle$ be the lattice defined using the subset ordering $\subseteq$. Then $\mathcal{L}_{\mathrm{B}}(s)=\left\langle\mathcal{P}\left(W_{\mathrm{B}}(s)\right)\right.$, $\rceil$ is isomorphic to $\langle\mathcal{P}(\operatorname{nodes}(\mathrm{B})), \subseteq\rangle$, where each $X \in \mathcal{P}\left(W_{\mathrm{B}}(s)\right)$ is mapped to the set of nodes that it contains.

Definition 4.4 $\left(\right.$ blocks $\left._{s}\right)$. Let $X \sqsubseteq W_{\mathrm{B}}(s)$ and $\mathrm{a}, \mathrm{B}_{i}$ be nodes in $W_{\mathrm{B}}(s)$. Then blocks $s_{s}(\mathrm{a}, X) \triangleq\left(\exists \mathrm{B}_{i} \in\right.$ $\left.X: \mathrm{a} \rightarrow \mathrm{B}_{i} \in W_{\mathrm{B}}(s)\right)$, and blocks $\left(\mathrm{B}_{i}, X\right) \triangleq\left(\forall \mathrm{a}: \mathrm{B}_{i} \rightarrow \mathrm{a} \in W_{\mathrm{B}}(s) \Rightarrow \mathrm{a} \in X\right)$.

Hence an interaction a is blocked by a set of nodes $X$ if some participant $\mathrm{B}_{i}$ of a is in $X$, and $\mathrm{B}_{i}$ does not enable a. A component $\mathrm{B}_{i}$ is blocked by $X$ if all of the interactions that $\mathrm{B}_{i}$ enables are in $X$. 
Definition $4.5\left(\mathcal{S}_{s}\right)$. Define $\mathcal{S}_{s}: \mathcal{P}\left(W_{\mathrm{B}}(s)\right) \rightarrow \mathcal{P}\left(W_{\mathrm{B}}(s)\right)$ as follows. $\mathcal{S}_{s}(X)$ is the subgraph with nodes $\left\{v \mid \operatorname{blocks}_{s}(v, X)\right\}$, together with their induced edges.

Definition $4.6\left(\mathcal{V}_{s}\right)$. Define $\mathcal{V}_{s}: \mathcal{P}\left(W_{\mathrm{B}}(s)\right) \rightarrow \mathcal{P}\left(W_{\mathrm{B}}(s)\right)$ as follows. $\mathcal{V}_{s}(X)$ is the subgraph with nodes $\{v \mid \neg$ blocks $(v, \bar{X})\}$, together with their induced edges.

Hence $\mathcal{V}_{s}(X)=\overline{\mathcal{S}_{s}(\bar{X})}$, i.e., $\mathcal{V}_{s}$ and $\mathcal{S}_{s}$ are duals. Note that $\mathcal{S}_{s}$ and $\mathcal{V}_{s}$ are defined given both a particular BIP system B and a particular state $s$ of B. Hence we should really write $\mathcal{S}_{\mathrm{B}, s}(X), \mathcal{V}_{\mathrm{B}, s}(X)$ to indicate this functional dependence. Since however, B is a fixed BIP-system, we omit the B subscript to avoid notational clutter. In giving examples, we usually omit the subscript for the state, since the state will be implicitly given by the example.

Proposition 4.7. $\mathcal{S}_{s}$ and $\mathcal{V}_{s}$ are monotone and continuous.

Proof. We show first that $\mathcal{S}_{s}$ is monotone, i.e., $X \sqsubseteq Y \Rightarrow \mathcal{S}_{s}(X) \sqsubseteq \mathcal{S}_{s}(Y)$. Let $v$ be an arbitrary node in $\mathcal{S}_{s}(X)$, so that blocks $_{s}(v, X)$ holds. There are two cases.

Case of $v$ is an interaction a. By Definitions 4.4 and 4.5, we have $\left(\exists \mathrm{B}_{i} \in X: \mathrm{a} \rightarrow \mathrm{B}_{i} \in W_{\mathrm{B}}(s)\right)$. Since $X \sqsubseteq Y$, this same $\mathrm{B}_{i}$ is also a node of $Y$, and so $\exists \mathrm{B}_{i} \in Y: \mathrm{a} \rightarrow \mathrm{B}_{i} \in W_{\mathrm{B}}(s)$. Hence blocks $_{s}(\mathrm{a}, Y)$, and so a $\in \mathcal{S}_{s}(Y)$.

Case of $v$ is a component $\mathrm{B}_{i}$. By Definitions 4.4 and 4.5 , we have $\left(\forall \mathrm{a}: \mathrm{B}_{i} \rightarrow \mathrm{a} \in W_{\mathrm{B}}(s) \Rightarrow \mathrm{a} \in X\right)$. Since $X \sqsubseteq Y$, we have $\left(\forall \mathrm{a}: \mathrm{B}_{i} \rightarrow \mathrm{a} \in W_{\mathrm{B}}(s) \Rightarrow \mathrm{a} \in Y\right)$. Hence blocks $s_{s}\left(\mathrm{~B}_{i}, Y\right)$, and so $\mathrm{B}_{i} \in \mathcal{S}_{s}(Y)$.

In both cases, we have $v \in \mathcal{S}_{s}(Y)$. Since $v$ was chosen arbitrarily from $\mathcal{S}_{s}(X)$, it follows that $\mathcal{S}_{s}(X) \sqsubseteq \mathcal{S}_{s}(Y)$. Hence $\mathcal{S}_{s}$ is monotone. Since the dual of a monotone mapping in a complete Boolean lattice is also monotone, we have that $\mathcal{V}_{s}$ is monotone. Finally, since $\mathcal{L}_{\mathrm{B}}(s)$ is finite, it follows that $\mathcal{S}_{s}$ and $\mathcal{V}_{s}$ are continuous.

Hence, by the Knaster-Tarski theorem, the least and greatest fixpoints of $\mathcal{S}_{s}$ and $\mathcal{V}_{s}$ exist.

Proposition 4.8. Let $X \neq \emptyset$ and $X \sqsubseteq W_{\mathrm{B}}(s)$, i.e., $X$ is a non-empty subgraph of $W_{\mathrm{B}}(s)$. Then $X$ is a supercycle in $W_{\mathrm{B}}(s)$ iff $X \sqsubseteq \mathcal{S}_{s}(X)$.

Proof. Let $X$ be a supercycle in $W_{\mathrm{B}}(s)$. By Definition 3.6, every node in $X$ is blocked by $X$, i.e., $\left(\forall x \in X: \operatorname{blocks}_{s}(x, X)\right)$. By Definition 4.5, $X \sqsubseteq \mathcal{S}_{s}(X)$. Conversely, suppose $X \sqsubseteq \mathcal{S}_{s}(X)$ for some subgraph $X$ of $W_{\mathrm{B}}(s)$. Hence $\left(\forall x \in X: x \in \mathcal{S}_{s}(X)\right)$, so by Definition 4.5, $\left(\forall x \in X:\right.$ blocks $\left._{s}(x, X)\right)$. Hence every node in $X$ is blocked by $X$, and so $X$ satisfies Definition 3.6, and is therefore a supercycle.

Thus the supercycles of $W_{\mathrm{B}}(s)$ are exactly the post-fixpoints of $\mathcal{S}$.

Proposition 4.9. Let $S C, S C^{\prime}$ be supercycles in $W_{\mathrm{B}}(s)$. Then $S C \sqcup S C^{\prime}$ is a supercycle in $W_{\mathrm{B}}(s)$.

Proof. By Proposition 4.8, $S C$ and $S C^{\prime}$ are post-fixpoints of $\mathcal{S}_{s}$. Since the join of post-fixpoints is a post-fixpoint, the proposition follows by applying Proposition 4.8 again.

Proposition 4.10. Let $S C$ be the greatest post-fixpoint of $\mathcal{S}_{s}$. Then either $(a) W_{\mathrm{B}}(s)$ is supercycle-free and $S C=\emptyset$, or $(b) W_{\mathrm{B}}(s)$ contains supercycles, and $S C$ is the largest supercycle in $W_{\mathrm{B}}(s)$.

Proof. By the Knaster-Tarski theorem, the greatest post-fixpoint is the join of all the postfixpoints. If $W_{\mathrm{B}}(s)$ is supercycle-free, then by Proposition 4.8, the only post-fixpoint of $\mathcal{S}_{s}$ is $\emptyset$ Hence $S C=\emptyset$. If $W_{\mathrm{B}}(s)$ contains supercycles, then by Proposition 4.8, the set of post-fixpoints of $\mathcal{S}_{s}$ is exactly the set of supercycles of $W_{\mathrm{B}}(s)$. Hence $S C$ is the join of all these supercycles. By Proposition 4.9, $S C$ is itself a supercycle. Hence $S C$ is the largest supercycle in $W_{\mathrm{B}}(s)$. 
Let $l f p, g f p$ denote the least fixpoint and greatest fixpoint operators, respectively.

Proposition 4.11. $v \in l f p\left(\mathcal{V}_{s}\right)$ iff $v$ is not a node in any supercycle of $W_{\mathrm{B}}(s)$.

Proof. From the Park conjugate (dual) fixpoint theorem in complete Boolean lattices [27], we have $l f p\left(\mathcal{V}_{s}\right)=\overline{g f p\left(\mathcal{S}_{s}\right)}$. By Proposition 4.10, $g f p\left(\mathcal{S}_{s}\right)$ is the largest supercycle in $W_{\mathrm{B}}(s)$. Hence the nodes not in $g f p\left(\mathcal{S}_{s}\right)$ are exactly the nodes that are not in any supercycle. These are exactly the nodes in $l f p\left(\mathcal{V}_{s}\right)$.

Define $\mathcal{V}_{s}^{1}(X)=\mathcal{V}_{s}(X)$, and for $d>1, \mathcal{V}_{s}^{d}(X)=\mathcal{V}_{s}\left(\mathcal{V}_{s}^{d-1}(X)\right)$, i.e., a superscript indicates functional iteration of $\mathcal{V}$. Also let $\sqcup$ be the "quantifier" version of $\sqcup$. Note that $\mathcal{V}_{s}^{d}(\emptyset) \sqsubseteq \mathcal{V}_{s}^{d^{\prime}}(\emptyset)$ when $d \leq d^{\prime}$, since $\mathcal{V}$ is monotone. Hence $\mathcal{V}_{s}^{1}(\emptyset), \mathcal{V}_{s}^{2}(\emptyset), \ldots$ is a non-decreasing sequence.

Proposition 4.12. $\operatorname{lfp}\left(\mathcal{V}_{s}\right)=\bigsqcup_{d \geq 1} \mathcal{V}_{s}^{d}(\emptyset)$.

Proof. By Proposition 4.7, $\mathcal{V}_{s}$ is continuous. Follows by standard results, e.g., see the CPO fixpoint theorem I in [20].

Definition 4.13 (Supercycle violation, $\operatorname{viol}_{\mathrm{B}}(v, s)$, $\operatorname{viol}_{\mathrm{B}}(v, s, d)$ ). Let $v$ be a node of $W_{\mathrm{B}}(s)$. Define $\operatorname{viol}_{\mathrm{B}}(v, s) \triangleq v \in l f p\left(\mathcal{V}_{s}\right)$ and, for $d \geq 1, \operatorname{viol}_{\mathrm{B}}(v, s, d) \triangleq v \in \mathcal{V}_{s}^{d}(\emptyset)$.

Proposition 4.14. $\operatorname{viol}_{\mathrm{B}}(v, s)$ iff $\left(\exists d \geq 1: \operatorname{viol}_{\mathrm{B}}(v, s, d)\right)$.

Proof. By Definition 4.13, viol $(v, s) \equiv v \in l f p\left(\mathcal{V}_{s}\right)$. By Proposition 4.12, $v \in \operatorname{lfp}\left(\mathcal{V}_{s}\right) \equiv$ $v \in \bigsqcup_{d \geq 1} \mathcal{V}_{s}^{d}(\emptyset)$. By Definition 4.13, $\left(\forall d \geq 1: \operatorname{viol}_{\mathrm{B}}(v, s, d) \equiv v \in \mathcal{V}_{s}^{d}(\emptyset)\right)$. Chaining these equivalences establishes the proposition.

It follows from Proposition 4.11 that $\operatorname{viol}_{\mathrm{B}}(v, s)$ iff there does not exist $S C$ such that $S C$ is a supercycle and $v \in S C$. We say that a node $v$ of $W_{\mathrm{B}}(s)$ has a supercycle violation iff $v$ is not a node in any supercycle of $W_{\mathrm{B}}(s)$, i.e., iff viol $\mathrm{B}_{\mathrm{B}}(v, s)$ holds. By Proposition 4.12, we can compute $l f p\left(\mathcal{V}_{s}\right)$ (and therefore $\operatorname{viol}_{\mathrm{B}}(v, s)$ ) by iterating $\mathcal{V}_{s}$, starting from $\emptyset$, until there is no more change. $\operatorname{viol}_{\mathrm{B}}(v, s, d)$ defines a supercycle violation that can be confirmed within $d$ iterations of $\mathcal{V}_{s}$, which we call a level-d supercycle violation. $\operatorname{viol}_{\mathrm{B}}(v, s)$ requires, in general, the entire least fixed point of $\mathcal{V}_{s}$.

Example 4.15 (Supercycle violation). For example, consider the wait-for graph in Figure 4. We show the set of nodes in each $\mathcal{V}^{d}(\emptyset)$, since the induced subgraph is easily inferred from Figure 4. $\mathcal{V}^{1}(\emptyset)=\{\mathrm{i}\}, \mathcal{V}^{2}(\emptyset)=\left\{\mathrm{B}_{6}, \mathrm{i}\right\}, \mathcal{V}^{3}(\emptyset)=\left\{\mathrm{h}, \mathrm{B}_{6}, \mathrm{i}\right\}, \mathcal{V}^{4}(\emptyset)=\left\{\mathrm{B}_{5}, \mathrm{~h}, \mathrm{~B}_{6}, \mathrm{i}\right\}, \mathcal{V}^{5}(\emptyset)=\left\{\mathrm{B}_{5}, \mathrm{~h}, \mathrm{~B}_{6}, \mathrm{i}\right\}$, as so $l f p(\mathcal{V})=\left\{B_{5}, h, B_{6}, i\right\}$. For Figure 5 , it is easy to verify that $l f p(\mathcal{V})$ consists of all the nodes in the system, i.e., the wait-for graph shown is supercycle-free.

Example 4.16 (Supercycle violations in dining philosophers). Figure 7 illustrates supercycle violations in four global states of the dining philosophers system of Figure 1. The states shown are the initial state, and the states resulting after execution of the indicated sequences of interactions. For each node $v$ (interaction or component), we include a small positive integer after its name, giving the smallest $d$ such that $v \in \mathcal{V}^{d}(\emptyset)$, i.e., the supercyle violation level.

Definition 4.17 (Supercycle membership, $s c y c_{\mathrm{B}}(v, s)$ ). Let $v$ be a node of $W_{\mathrm{B}}(s)$. Then $s c y c_{\mathrm{B}}(v, s)$ holds iff there exists a supercycle $S C \sqsubseteq W_{\mathrm{B}}(s)$ such that $v \in S C$.

Proposition 4.18. Let $v$ be a node of $W_{\mathrm{B}}(s)$. Then $\neg s c y c_{\mathrm{B}}(v, s)$ iff $\operatorname{viol}_{\mathrm{B}}(v, s)$ That is, a node is not in any supercycle iff it has a supercycle violation.

Proof. Immediate from Definition 4.13, Definition 4.17, and Proposition 4.11.

\footnotetext{
${ }^{1}$ Note that we abuse notation by overloading viol, but no ambiguity arises since the two versions have different parameter lists.
} 


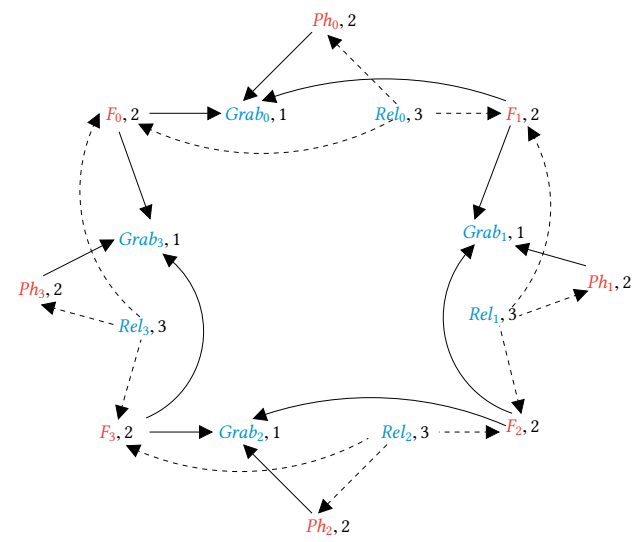

(a) Supercycle violations in initial state.

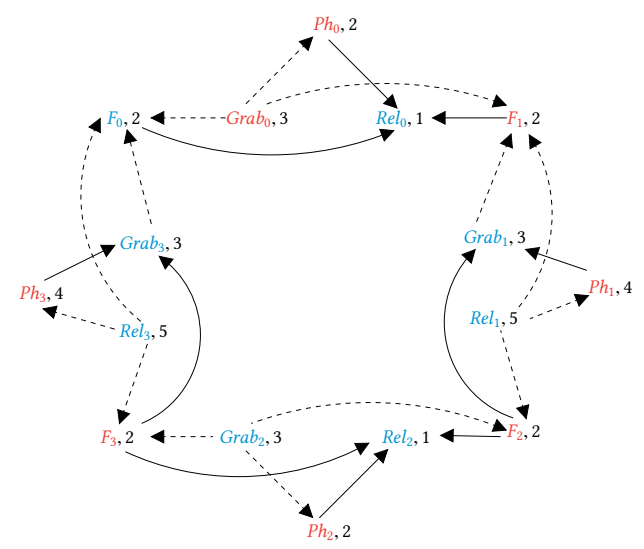

(c) Supercycle violations after execution of $\mathrm{Grab}_{0} ; \mathrm{Grab}_{2}$.

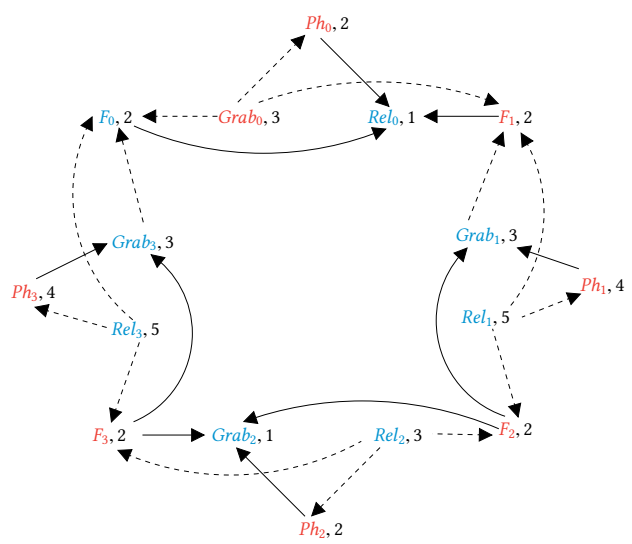

(b) Supercycle violations after execution of $\mathrm{Grab}_{0}$.

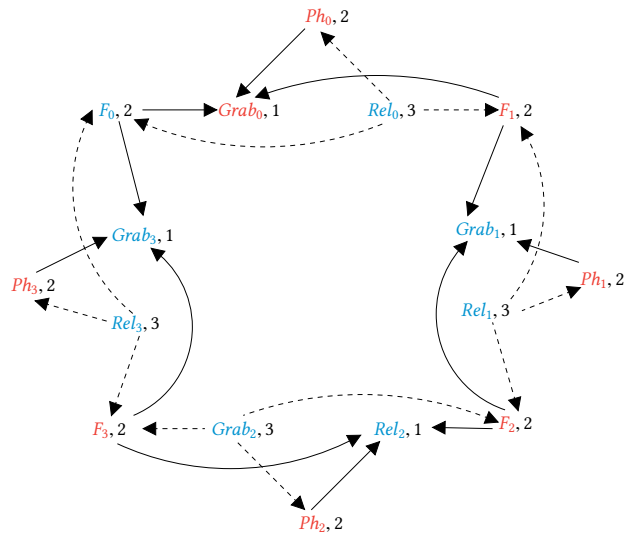

(d) Supercycle violations after execution of $\mathrm{Grab}_{0} ; \mathrm{Grab}_{2} ; \mathrm{Rel}_{0}$.

Fig. 7. Example supercycle violations for dining philosophers system of Figure 1.

\subsection{Structural properties of supercycles}

We present some structural properties of supercycles, which are central to our deadlock-freedom conditions.

Define $\operatorname{preds}_{\mathrm{B}}(v, s)=\left\{w \mid w \rightarrow v \in W_{\mathrm{B}}(s)\right\}$ and $\operatorname{succs}_{\mathrm{B}}(v, s)=\left\{w \mid v \rightarrow w \in W_{\mathrm{B}}(s)\right\}$. The definition of a supercycle (Definition 3.6) imposes certain constraints on supercycle membership of a node w.r.t. its predecessors and successors in the wait-for-graph, as follows:

Proposition 4.19 (Supercycle membership constraints). Let $\mathrm{a}, \mathrm{B}_{i}$ be nodes of $W_{\mathrm{B}}(s)$. Then

(1) $s c y c_{\mathrm{B}}\left(\mathrm{B}_{i}, s\right) \equiv\left(\forall \mathrm{a} \in \operatorname{succs}_{\mathrm{B}}\left(\mathrm{B}_{i}, s\right): s c y c_{\mathrm{B}}(\mathrm{a}, s)\right)$.

(2) $\operatorname{scyc}_{\mathrm{B}}\left(\mathrm{B}_{i}, s\right) \Rightarrow\left(\forall \mathrm{a} \in \operatorname{preds}_{\mathrm{B}}\left(\mathrm{B}_{i}, s\right): s c y c_{\mathrm{B}}(\mathrm{a}, s)\right)$.

(3) $s c y c_{\mathrm{B}}(\mathrm{a}, s) \equiv\left(\exists \mathrm{B}_{i} \in \operatorname{succs}_{\mathrm{B}}(\mathrm{a}, s): s c y c_{\mathrm{B}}\left(\mathrm{B}_{i}, s\right)\right)$.

(4) $\operatorname{scyc}_{\mathrm{B}}(\mathrm{a}, s) \Leftarrow\left(\exists \mathrm{B}_{i} \in \operatorname{preds}_{\mathrm{B}}(\mathrm{a}, s): \operatorname{scyc}_{\mathrm{B}}\left(\mathrm{B}_{i}, s\right)\right)$.

Proof. We deal with each clause in turn. 
Proof of Clause 1. Assume $s c y c_{\mathrm{B}}\left(\mathrm{B}_{i}, s\right)$, and let $S C \sqsubseteq W_{\mathrm{B}}(s)$ be the supercycle containing $\mathrm{B}_{i}$. By Definition 3.6, Clause 2, $\left(\forall \mathrm{a} \in \operatorname{succs}_{\mathrm{B}}\left(\mathrm{B}_{i}, s\right): \operatorname{scyc}_{\mathrm{B}}(\mathrm{a}, s)\right)$. We conclude $\operatorname{scyc}_{\mathrm{B}}\left(\mathrm{B}_{i}, s\right) \Rightarrow(\forall \mathrm{a} \in$ $\left.\operatorname{succs}_{\mathrm{B}}\left(\mathrm{B}_{i}, s\right): \operatorname{scyc}_{\mathrm{B}}(\mathrm{a}, s)\right)$. Now assume $\left(\forall \mathrm{a} \in \operatorname{succs}_{\mathrm{B}}\left(\mathrm{B}_{i}, s\right): \operatorname{scyc}_{\mathrm{B}}(\mathrm{a}, s)\right)$, and let $S C$ be the join of all the supercycles containing all the a $\in \operatorname{succs}_{\mathrm{B}}\left(\mathrm{B}_{i}, s\right)$. By Proposition 4.9, $S C \sqsubseteq W_{\mathrm{B}}(s)$ is a supercycle. Let $S C^{\prime}$ be $S C$ with edge $\mathrm{B}_{i} \rightarrow$ a added, for all a $\in \operatorname{succs}_{\mathrm{B}}\left(\mathrm{B}_{i}, s\right)$. Then $S C^{\prime}$ is a supercycle by Definition 3.6, and also $S C^{\prime} \sqsubseteq W_{\mathrm{B}}(s)$. Hence $s c y c_{\mathrm{B}}(\mathrm{a}, s)$. We conclude $s c y c_{\mathrm{B}}\left(\mathrm{B}_{i}, s\right) \Leftarrow(\forall \mathrm{a} \in$ $\left.\operatorname{succs}_{\mathrm{B}}\left(\mathrm{B}_{i}, s\right): s c y c_{\mathrm{B}}(\mathrm{a}, s)\right)$.

Proof of Clause 2. Assume $s c y c_{\mathrm{B}}\left(\mathrm{B}_{i}, s\right)$, so that $S C \sqsubseteq W_{\mathrm{B}}(s)$ is the supercycle containing $\mathrm{B}_{i}$. Let $\mathrm{a} \in \operatorname{preds}_{\mathrm{B}}\left(\mathrm{B}_{i}, s\right)$, and let $S C^{\prime}$ be $S C$ with a $\rightarrow \mathrm{B}_{i}$ added. Hence $S C^{\prime}$ is a supercycle by Definition 3.6, Clause 3. Since a was chosen arbitrarily, we conclude $\left(\forall \mathrm{a} \in \operatorname{pred}_{\mathrm{B}}\left(\mathrm{B}_{i}, s\right): s c y c_{\mathrm{B}}(\mathrm{a}, s)\right)$.

Proof of Clause 3. Assume $s c y c_{\mathrm{B}}(\mathrm{a}, s)$, and let $S C \sqsubseteq W_{\mathrm{B}}(s)$ be the supercycle containing a. By Definition 3.6, Clause 3, there exists some $\mathrm{B}_{i} \in \operatorname{succs}_{\mathrm{B}}(\mathrm{a}, s)$ such that $\mathrm{B}_{i} \in S C$. Hence $s c y c_{\mathrm{B}}\left(\mathrm{B}_{i}, s\right)$. We conclude $s c y c_{\mathrm{B}}(\mathrm{a}, s) \Rightarrow\left(\exists \mathrm{B}_{i} \in \operatorname{succs}_{\mathrm{B}}(\mathrm{a}, s): \operatorname{scyc}_{\mathrm{B}}\left(\mathrm{B}_{i}, s\right)\right)$. Now assume $\left(\exists \mathrm{B}_{i} \in \operatorname{succs}_{\mathrm{B}}(\mathrm{a}, s):\right.$ $\left.s c y c_{\mathrm{B}}\left(\mathrm{B}_{i}, s\right)\right)$, and let $S C \sqsubseteq W_{\mathrm{B}}(s)$ be the supercycle containing some $\mathrm{B}_{i} \in \operatorname{succs}_{\mathrm{B}}(\mathrm{a}, s)$. Let $S C^{\prime}$ be $S C$ with a $\rightarrow \mathrm{B}_{i}$ added. Then $S C^{\prime}$ is a supercycle by Definition 3.6, and also $S C^{\prime} \sqsubseteq W_{\mathrm{B}}(s)$. Hence $s c y c_{\mathrm{B}}(\mathrm{a}, s)$. We conclude $s c y c_{\mathrm{B}}(\mathrm{a}, s) \Leftarrow\left(\exists \mathrm{B}_{i} \in \operatorname{succs}_{\mathrm{B}}(\mathrm{a}, s): s c y c_{\mathrm{B}}\left(\mathrm{B}_{i}, s\right)\right)$.

Proof of Clause 4. Assume $\neg s c y c_{\mathrm{B}}(\mathrm{a}, s)$, so that a is not in any supercycle of $W_{\mathrm{B}}(s)$. Let $\mathrm{B}_{i} \in$ preds $s_{\mathrm{B}}(\mathrm{a}, s)$. By Definition 3.6, Clause 2, $\mathrm{B}_{i}$ cannot be in any supercycle of $W_{\mathrm{B}}(s)$, since all aa $\in$ $\operatorname{succs}_{\mathrm{B}}\left(\mathrm{B}_{i}, s\right)$ must also be in the supercycle. Hence $\neg s c y c_{\mathrm{B}}\left(\mathrm{B}_{i}, s\right)$. Since $\mathrm{B}_{i}$ was chosen arbitrarily, we conclude $\neg s c y c_{\mathrm{B}}(\mathrm{a}, s) \Rightarrow\left(\forall \mathrm{B}_{i} \in \operatorname{preds}_{\mathrm{B}}(\mathrm{a}, s): \neg s c y c_{\mathrm{B}}\left(\mathrm{B}_{i}, s\right)\right)$, the contrapositive of Clause 4 .

Note that Clause 2 cannot be strengthened to an equivalence: if all the interactions that wait for a component $\mathrm{B}_{i}$ are in a supercycle, then $\mathrm{B}_{i}$ itself may or may not be in a supercycle, depending on whether $B_{i}$ is waiting for some other interaction aa that is not in a supercycle. Likewise, Clause 4 cannot be strengthened to an equivalence: if $\mathrm{a}$ is in a supercycle, then any component $\mathrm{B}_{i}$ that waits for a may or may not be in a supercycle, depending on whether $\mathrm{B}_{i}$ is waiting for some other interaction aa that is not in a supercycle.

While Proposition 4.19 gives relationships between supercycle membership of a node and both its successors and predecessors, nevertheless Definition 3.6 implies that the "causality" of supercyclemembership of a node $v$ is from the successors of $v$ to $v$, i.e., membership of $v$ in a supercycle is caused only by membership of $v$ 's successors in a supercycle. Repeating this step, we infer that $v$ 's supercycle-membership is caused by the subgraph of the wait-for graph that is reachable from $v$.

Proposition 4.20. Every supercycle SC contains at least two nodes.

Proof. By Definition 3.6, SC is nonempty, and so contains at least one node $v$. If $v$ is an interaction a, then by Definition 3.6, SC also contains some component $\mathrm{B}_{i}$ such that $\mathrm{a} \rightarrow \mathrm{B}_{i}$. If $v$ is a component $\mathrm{B}_{i}$, then, by assumption, $\mathrm{B}_{i}$ enables at least one interaction a, and by Definition 3.6, every interaction that $\mathrm{B}_{i}$ enables must be in $S C$. Hence in both cases, $S C$ contains at least two nodes.

Proposition 4.21. Every supercycle SC contains a maximal strongly connected component CC such that (1) CC is itself a supercycle, and (2) there is no wait-for edge from a node in CC to a node outside of CC.

Proof. SC is a directed graph, and so consider the decomposition of $S C$ into its maximal strongly connected components (MSCC). Let $\operatorname{mscc}(S C)$ be the graph resulting from replacing each MSCC by a single node. By its construction, $\operatorname{mscc}(S C)$ is acyclic, and so contains at least one node $x$ with no outgoing edges. Let $C C$ be the MSCC corresponding to $x$. It follows from the construction of $C C$ that no node in $C C$ has a wait-for edge going to a node outside of $C C$, and so Clause (2) of the Proposition is established. 
It also follows from the construction of $C C$ that $C C$ is nonempty, and hence $C C$ satisfies clause (1) of Definition 3.6. Let $v$ be an arbitrary node in $C C$. Since $C C \sqsubseteq S C, v$ is a node of $S C$. Let $w$ be an arbitrary successor of $v$ in $S C$. Since no node in $C C$ has an edge going to a node outside of $C C$, it follows that $w$ is a node of $C C$. Hence $v$ has the same successors in $C C$ as in $S C$. Now since $S C$ is a supercycle, every vertex $v$ in $S C$ has enough successors in $S C$ to satisfy clauses (2) and (3) of Definition 3.6. It follows that every vertex $v$ in $C C$ has enough successors in $C C$ to satisfy clauses (2) and (3) of Definition 3.6. Hence, by Definition 3.6, CC is itself a supercycle, and so Clause (1) of the Proposition is established.

Note also that by Proposition 4.20, $C C$ contains at least two nodes. Hence $C C$ is not a trivial strongly connected component.

Definition 4.22 (Path, path length). Let $G$ be a directed graph and $v$ a vertex in $G$. A path $\pi$ in $G$ is a finite sequence $v_{0}, v_{1}, \ldots, v_{n}$ such that $\left(v_{i}, v_{i+1}\right)$ is an edge in $G$ for all $i \in\{0, \ldots, n-1\}$. Write path $_{G}(\pi)$ iff $\pi$ is a path in $G$. Define $\operatorname{first}(\pi)=v_{0}$ and last $(\pi)=v_{n}$. Let $|\pi|$ denote the length of $\pi$, which we define as follows:

- if $\pi$ is simple, i.e., all $v_{i}, 0 \leq i \leq n$, are distinct, then $|\pi|=n$, i.e., the number of edges in $\pi$

- if $\pi$ contains a cycle, i.e., there exist $v_{i}, v_{j}$ such that $i \neq j$ and $v_{i}=v_{j}$, then $|\pi|=\omega(\omega$ for "infinity").

Definition 4.23 (In-depth, out-depth). Let $G$ be a directed graph and $v$ a vertex in $G$. Define the in-depth of $v$ in $G$, notated as in_depth ${ }_{G}(v)$, as follows:

- if there exists a path $\pi$ in $G$ that contains a cycle and ends in $v$, i.e., $|\pi|=\omega \wedge \operatorname{last}(\pi)=v$, then in_depth $_{G}(v)=\omega$,

- otherwise, let $\pi$ be a longest (simple) path ending in $v$. Then in_depth ${ }_{G}(v)=|\pi|$.

Formally, in_depth ${ }_{G}(v)=\left(\operatorname{MAX} \pi: \operatorname{path}_{G}(\pi) \wedge \operatorname{last}(\pi)=v:|\pi|\right)$.

Likewise define the out-depth of $v$ in $G$, notated as out_depth $h_{G}(v)$, as follows:

- if there exists a path $\pi$ in $G$ that contains a cycle and starts in $v$, i.e., $|\pi|=\omega \wedge \operatorname{first}(\pi)=v$, then out_depth $(v)=\omega$,

- otherwise, let $\pi$ be a longest (simple) path starting in $v$. Then out_depth $(v)=|\pi|$.

Formally, out_depth $(v)=\left(\operatorname{MAX} \pi: \operatorname{path}_{G}(\pi) \wedge \operatorname{first}(\pi)=v:|\pi|\right)$.

We use in_depth $(v, s)$ for in_depth $h_{W_{\mathrm{B}}(s)}(v)$, and also out_depth $(v, s)$ for out_depth $h_{W_{\mathrm{B}}(s)}(v)$. A node with finite in-depth is not reachable from any non-trivial (i.e., consisting of more than one node) MSCC, and a node with finite out-depth cannot reach any non-trivial MSCC.

Proposition 4.24. Assume that node $v$ of $W_{\mathrm{B}}(s)$ has a finite out-depth of $d \geq 0$ in $W_{\mathrm{B}}(s)$, i.e., out_depth $h_{\mathrm{B}}(v, s)=d$. Then $\operatorname{viol}_{\mathrm{B}}(v, s, d+1)$.

Proof. Proof is by induction on $d$.

Base case, $d=0$. Hence by out_depth $(v, s)=0$ and Definitions 4.22 and 4.23 , $v$ has no outgoing wait-for edges in $W_{\mathrm{B}}(s)$. Hence $\neg$ blocks $s_{s}\left(v, W_{\mathrm{B}}(s)\right)$, i.e., $v$ is not blocked by the entire set of nodes in $W_{\mathrm{B}}(s)$. Hence $\neg$ blocks $(v, \bar{\emptyset})$, since $W_{\mathrm{B}}(s)=\bar{\emptyset}$. So by Definition 4.6, $v \in \mathcal{V}_{s}(\emptyset)$. By Definition 4.13, $\operatorname{viol}_{\mathrm{B}}(v, s, 1)$.

Inductive step, $d>0$. Let $w$ be an arbitrary successor of $v$, i.e., a node $w$ such that $v \rightarrow w \in W_{\mathrm{B}}(s)$. By Definitions 4.22 and $4.23, w$ has an out-depth $d^{\prime}$ that is less than $d$. That is, out_depth $(u, s)=$ $d^{\prime}<d$. By the induction hypothesis applied to $d^{\prime}$, we obtain viol $\mathrm{B}_{\mathrm{B}}\left(w, s, d^{\prime}+1\right)$, and so $w \in \mathcal{V}_{s}^{d^{\prime}+1}(\emptyset)$ by Definition 4.13. Hence $w \in \mathcal{V}_{s}^{d}(\emptyset)$, since, by monotonicity of $\mathcal{V}_{s}$, we have $\mathcal{V}_{s}^{n^{\prime}}(\emptyset) \sqsubseteq \mathcal{V}_{s}^{n}(\emptyset)$ 
when $n^{\prime} \leq n$. Since $w$ is an arbitrary successor of $v$, it follows that $v$ is only blocked by nodes in $\mathcal{V}_{s}^{d}(\emptyset)$. Hence $\neg$ blocks $\left(v, \overline{\mathcal{V}_{s}^{d}(\emptyset)}\right)$. By Definition 4.6, $v \in \mathcal{V}_{s}\left(\mathcal{V}_{s}^{d}(\emptyset)\right)$, i.e., $v \in \mathcal{V}_{s}^{d+1}(\emptyset)$. By Definition 4.13, $\operatorname{viol}_{\mathrm{B}}(v, s, d+1)$.

COROLlary 4.25. A supercycle SC contains no nodes with finite out-depth.

Proof. Let $v$ be a node in $S C$ with finite out-depth $d$. By Proposition 4.24, $\operatorname{viol}_{\mathrm{B}}(v, s, d+1)$. By Definition 4.13, $\operatorname{viol}_{\mathrm{B}}(v, s)$. By Proposition $4.18 \neg s c y c_{\mathrm{B}}(v, t)$. Hence $v$ cannot be a node of any supercycle, and we have a contradiction.

Proposition 4.26. Every supercycle SC contains at least one cycle.

Proof. By contradiction. Suppose that $S C$ is a supercycle and is also acyclic. Then every path in $S C$ is simple, and therefore finite. Hence every node in $S C$ has finite out-depth. By Proposition 4.24, $S C$ cannot be a supercycle.

Proposition 4.27. Let $S C$ be a supercycle in $W_{\mathrm{B}}(s)$, and let $S C^{\prime}$ be the graph obtained from $S C$ by removing all vertices of finite in-depth and their incident edges. Then $S C^{\prime}$ is also a supercycle in $W_{\mathrm{B}}(s)$.

Proof. A vertex with finite in-depth cannot lie on a cycle in SC. Hence by Proposition 4.26, $S C^{\prime} \neq \emptyset$. Thus $S C^{\prime}$ satisfies clause (1) of the supercycle definition (3.6). Let $v$ be an arbitrary vertex of $S C^{\prime}$. Thus $v \in S C$ and in_depth $h_{S C}(v)=\omega$ by definition of $S C^{\prime}$. Let $w$ be an arbitrary successor of $v$ in $S C$, i.e., $v \rightarrow w \in S C$. Hence in_depth ${ }_{S C}(w)=\omega$ by Definition 4.23 . Hence $w \in S C^{\prime}$, by definition of $S C^{\prime}$. Furthermore, $v \rightarrow w \in S C^{\prime}$, since $S C^{\prime}$ contains all nodes of $S C$ with infinite in-depth. Hence the successors of $v$ in $S C^{\prime}$ are the same as the successors of $v$ in $S C$ Now since $S C$ is a supercycle, every vertex $v$ in $S C$ has enough successors in $S C$ to satisfy clauses (2) and (3) of the supercycle definition (3.6). It follows that every vertex $v$ in $S C^{\prime}$ has enough successors in $S C^{\prime}$ to satisfy clauses (2) and (3) of the supercycle definition (3.6). Hence $S C^{\prime}$ is a supercycle in $W_{\mathrm{B}}(s)$.

\section{GLOBAL CONDITIONS FOR DEADLOCK FREEDOM}

\subsection{The supercycle formation condition}

We use the structural properties of supercycles (Section 4.2) and the dynamics of wait-for graphs (Proposition 3.5) to define a condition that must hold whenever a supercycle is created. Negating this condition then implies the absence of supercycles.

Proposition 5.1 (Supercycle formation COndition). Assume that $t \stackrel{\text { a }}{\rightarrow} s$ is a transition of BIP-composite component $\mathrm{B}=\gamma\left(\mathrm{B}_{1}, \ldots, \mathrm{B}_{n}\right), W_{\mathrm{B}}(t)$ is supercycle-free, and that $W_{\mathrm{B}}(s)$ contains a supercycle. Then, in $W_{\mathrm{B}}(s)$, there exists a $C C$ such that

(1) $C C$ is a subgraph of $W_{\mathrm{B}}(s)$, i.e., $C C \sqsubseteq W_{\mathrm{B}}(s)$,

(2) $C C$ is strongly connected,

(3) CC is a supercycle,

(4) in $W_{\mathrm{B}}(s)$, there is no wait-for edge from a node in CC to a node outside of CC, and

(5) there exists a component $\mathrm{B}_{i} \in$ components(a) such that $\mathrm{B}_{i}$ is in $C C$.

Proof. By assumption, there is a supercycle $S C$ that is a subgraph of $W_{\mathrm{B}}(s)$. By Proposition 4.21, $S C$ contains a subgraph $C C$ that is strongly connected, is itself a supercycle, and such that there is no wait-for-edge from a node in $C C$ to a node outside of $C C$. This establishes Clauses 1-4.

Now suppose $\mathrm{B}_{i} \notin C C$ for every $\mathrm{B}_{i} \in$ components $(\mathrm{a})$. Then, no edge in $C C$ is $\mathrm{B}_{i}$-incident. Hence, by Proposition 3.5, every edge in $C C$ is an edge in $W_{\mathrm{B}}(t)$. Hence $C C$ is a subgraph of $W_{\mathrm{B}}(t)$. Now let $v$ be an arbitrary node in $C C$. Suppose $v$ is a component $\mathrm{B}_{j}$. By assumption, $\mathrm{B}_{j} \notin$ components $(\mathrm{a})$, and so $s\left\lceil\mathrm{~B}_{j}=t\left\lceil\mathrm{~B}_{j}\right.\right.$ by Definition 2.3. Hence $\mathrm{B}_{j}$ enables the same set of interactions in state $t$ as 
in state $s$. Also, in $W_{\mathrm{B}}(s)$, all of $\mathrm{B}_{j}$ 's wait-for edges must end in an interaction that is in $C C$, since $C C$ is a supercycle in $W_{\mathrm{B}}(s)$. Hence the same holds in $W_{\mathrm{B}}(t)$. If $v$ is an interaction, it must have a wait-for edge $e^{\prime}$ to some component $\mathrm{B}_{j} \in C C$, since $C C$ is a supercycle in $W_{\mathrm{B}}(s)$. Hence this also holds in $W_{\mathrm{B}}(t)$, since $s\left\lceil\mathrm{~B}_{j}=t\left\lceil\mathrm{~B}_{j}\right.\right.$. Hence $v$ has enough successors in $C C$ to satisfy the supercycle definition (Definition 3.6). We conclude that $C C$ by itself is a supercycle in $W_{\mathrm{B}}(t)$, which contradicts the assumption that $W_{\mathrm{B}}(t)$ is supercycle-free. Hence, $\mathrm{B}_{i} \in C C$ for some $\mathrm{B}_{i} \in \operatorname{components}(\mathrm{a})$, and so Clause 5 is established.

The supercycle formation condition (Proposition 5.1) tells us that, when a supercycle $S C$ is created, some component $\mathrm{B}_{i}$ that participates in the interaction a whose execution created $S C$, must be a node of a strongly connected component $C C$ of $S C$, and moreover $C C$ is itself a supercycle in its own right. In a sense, $C C$ is the "essential" part of $S C$. We use this to formulate a condition that prevents the formation of supercycles. For transition $t \stackrel{\mathrm{a}}{\rightarrow} s$, we determine for every component $\mathrm{B}_{i} \in$ components(a) whether it is possible for $\mathrm{B}_{i}$ to be a node in a strongly-connected supercycle $C C$ in $W_{\mathrm{B}}(s)$. There are two ways for $\mathrm{B}_{i}$ to not be a node in a strongly-connected supercycle:

(1) no supercycle membership: $\mathrm{B}_{i}$ is not a node of any supercycle, i.e., $\neg s c y c_{\mathrm{B}}\left(\mathrm{B}_{i}, s\right)$.

(2) no strong-connectedness: $\mathrm{B}_{i}$ is a node in a supercycle, but not a node in a strongly-connected supercycle.

Hence, for a BIP system (B, $\left.Q_{0}\right)$, our fundamental criterion for the prevention of supercycles is that, for every reachable transition $t \stackrel{\text { a }}{\rightarrow} s$ resulting from execution of a, in the resulting state $s$, every component $\mathrm{B}_{i}$ of a must violate at least one of the above two conditions. Condition (1) is just supercycle violation, as in Definition 4.13. Condition (2) is violation with respect to a strongly connected supercycle, i.e., non-membership in a strongly connected supercycle. Technically, this is implied by supercycle violation, and so the disjunction of the two conditions is equivalent to Condition (1). It is however, convenient to use the disjunction of the two conditions, since we will formulate local versions of these violation conditions, and the implication does not necessarily hold for the local versions.

For a given BIP system (B, $\left.Q_{0}\right)$ and interaction a, we use $\mathcal{G} \mathcal{A} \mathcal{L} \mathcal{T}\left(\mathrm{B}, Q_{0}\right.$, a) to denote the deadlockfreedom criterion based on the disjunction of Conditions (1) and (2) above. This criterion is, in a sense, the "most general" criterion for supercycle-freedom. If $\mathcal{G} \mathcal{A} \mathcal{L} \mathcal{T}\left(\mathrm{B}, Q_{0}\right.$, a) holds, global state $t$ is supercycle-free, and $t \stackrel{\text { a }}{\rightarrow} s$, then it follows (as we establish below) that global state $s$ is also supercycle-free. So, by requiring (1) that all initial states are supercycle-free, and (2) that $\mathcal{G} \mathcal{A} \mathcal{L} \mathcal{T}\left(\mathrm{B}, Q_{0}, \mathrm{a}\right)$ holds for all interactions a $\in \gamma$, we obtain, by straightforward induction on length of executions, that every reachable state is supercycle-free.

It also follows that any condition which implies $\mathcal{G} \mathcal{A} \mathcal{L} \mathcal{T}\left(\mathrm{B}, Q_{0}\right.$, a) is also sufficient to guarantee supercycle-freedom, and hence deadlock-freedom. We exploit this in two ways:

(1) To define a "linear" condition, $G \mathcal{L} \mathcal{I}$, that is easier to evaluate than $\mathcal{G} \mathcal{A} \mathcal{L}$, since it requires only the evaluation of lengths of wait-for paths, i.e., it does not have the "alternating" character of $\mathcal{G} \mathcal{A} \mathcal{L} \mathcal{T}$. It also implies $\mathcal{G} \mathcal{A} \mathcal{L} \mathcal{T}$.

(2) To define "local variants" of $\mathcal{G} \mathcal{A} \mathcal{L} \mathcal{T}$ and $\mathcal{G} \mathcal{L} \mathcal{N}$, which we denote as $\mathcal{L} \mathcal{A} \mathcal{L} \mathcal{T}$ and $\mathcal{L} \mathcal{L} \mathcal{N}$, respectively. $\mathcal{L} \mathcal{A} \mathcal{L} \mathcal{T}$ and $\mathcal{L} \mathcal{L} \mathcal{I} N$ can be evaluated in small subsystems of $\left(\mathrm{B}, Q_{0}\right)$. When either $\mathcal{L} \mathcal{A} \mathcal{L} \mathcal{T}$ or $\mathcal{L} \mathcal{L} \mathcal{I}$ holds in a small subsystem, we confirm deadlock freedom of (B, $Q_{0}$ ) without state-explosion. The local conditions imply the corresponding global ones, i.e., they are sufficient but not necessary for deadlock-freedom.

We therefore now have four deadlock-freedom conditions: $\mathcal{G} \mathcal{A} \mathcal{L} \mathcal{T}$ (global alternating), $\mathcal{L} \mathcal{A} \mathcal{L} \mathcal{T}$ (local alternating), $\mathcal{G} \mathcal{L} \mathcal{N}$ (global linear), and $\mathcal{L} \mathcal{L} \mathcal{I N}$ (local linear). These are all concrete instances of the abstract version of the deadlock-freedom condition given in Section 3.4. 


\subsection{A global AND-OR condition for deadlock-freedom}

We wish to show that the transition $t \stackrel{\text { a }}{\rightarrow} s$ does not create a supercycle in state $s$. Towards this end, we first formalize violation of strong connectedness (Condition 2 above) as follows.

Definition 5.2 (Strong connectedness violation, $\mathrm{sConnViol}_{\mathrm{B}}(v, s)$ ). Let $v$ be a node of $W_{\mathrm{B}}(s)$. Then ${ }_{\mathrm{SConnViol}}(v, s)$ holds iff there does not exist a strongly connected supercycle $S S C$ such that $v \in S S C$ and $S S C \sqsubseteq W_{\mathrm{B}}(s)$.

The general supercycle violation condition is then a disjunction of the supercycle violation condition and the strong connectedness violation conditions.

Definition 5.3 (General supercycle violation, $\operatorname{genViol} \mathrm{B}_{\mathrm{B}}(v, s)$ ). Let $v$ be a node of $W_{\mathrm{B}}(s)$. Then $\operatorname{genViol}_{\mathrm{B}}(v, s) \triangleq \operatorname{viol}_{\mathrm{B}}(v, s) \vee \mathrm{sConnViol}_{\mathrm{B}}(v, s)$.

Let $t \stackrel{\mathrm{a}}{\rightarrow} s$ be a reachable transition. If, for every $\mathrm{B}_{i} \in \operatorname{components}(\mathrm{a})$, genViol $\mathrm{B}(v, s)$ holds, then, as we show below, $t \stackrel{\text { a }}{\rightarrow} s$ does not introduce a supercycle, i.e., if $t$ is supercycle-free, then so is $s$. As stated above, we formulate below a "local" version of the general condition, which can be evaluated in "small subsystems", and so we often avoid state-explosion. We reiterate that viol ${ }_{\mathrm{B}}(v, s)$ implies that $v$ cannot be in a supercycle. Hence, $v$ cannot be in a strongly-connected supercycle. Hence $\operatorname{viol}_{\mathrm{B}}(v, s) \Rightarrow \operatorname{sConnViol}_{\mathrm{B}}(v, s)$, so that $\operatorname{viol}_{\mathrm{B}}(v, s) \vee \mathrm{sConnViol}_{\mathrm{B}}(v, s) \equiv \operatorname{sConnViol}_{\mathrm{B}}(v, s)$. We give the formation violation condition in this manner, since the implication does not necessarily hold for the local versions of $\operatorname{viol}_{\mathrm{B}}(v, s)$ and $\mathrm{sConnViol}_{\mathrm{B}}(v, s)$.

This discussion leads to the formal definition of $\mathcal{G} \mathcal{A} \mathcal{L} \mathcal{T}$ : after execution of interaction a, all $\mathrm{B}_{i} \in$ components (a) exhibit a general supercycle-violation, as given by gen $\mathrm{Viol}_{\mathrm{B}}\left(\mathrm{B}_{i}, s\right)$ above.

Definition $5.4\left(\mathcal{G} \mathcal{A} \mathcal{L} \mathcal{T}\left(\mathrm{B}, Q_{0}, \mathrm{a}\right)\right)$. Let $t \stackrel{\mathrm{a}}{\rightarrow} s$ be a reachable transition of $\left(\mathrm{B}, Q_{0}\right)$. Then, for every component $\mathrm{B}_{i} \in$ components $(\mathrm{a})$, the formation violation condition holds in state $s$. Formally,

$\forall \mathrm{B}_{i} \in$ components $(\mathrm{a})$, genViol $_{\mathrm{B}}\left(\mathrm{B}_{i}, s\right)$.

Theorem 5.5. $\mathcal{G} \mathcal{A} \mathcal{L} \mathcal{T}$ is supercycle-freedom preserving.

Proof. We establish: for every reachable transition $t \stackrel{\mathrm{a}}{\rightarrow} s, W_{\mathrm{B}}(t)$ is supercycle-free implies that $W_{\mathrm{B}}(s)$ is supercycle-free. Our proof is by contradiction, so we assume the existence of a reachable transition $t \stackrel{\text { a }}{\rightarrow} s$ such that $W_{\mathrm{B}}(t)$ is supercycle-free and $W_{\mathrm{B}}(s)$ contains a supercycle. By Proposition 5.1 there exists a component $\mathrm{B}_{i} \in$ components $(\mathrm{a})$ such that $\mathrm{B}_{i}$ is in $C C$, where $C C$ is a strongly connected supercycle that is a subgraph of $W_{\mathrm{B}}(s)$. Since $C C$ is a strongly connected supercycle, we have, by Definition 5.2, that $\neg \mathrm{sConnViol} \mathrm{B}_{\mathrm{B}}\left(\mathrm{B}_{i}, s\right)$ holds. Since $C C$ is a supercycle, we have, by Proposition 4.18, that $\neg \operatorname{viol}_{\mathrm{B}}\left(\mathrm{B}_{i}, s\right)$ holds. Hence, by Definition $5.3, \neg$ genViol $\left(\mathrm{B}_{i}, s\right)$. But, by Definition 5.4, we have genViol ${ }_{\mathrm{B}}\left(\mathrm{B}_{i}, s\right)$. Hence, we have the desired contradiction, and so the theorem holds.

\subsection{A global linear condition for deadlock-freedom}

In some cases, a simpler condition suffices to guarantee deadlock-freedom. This simpler condition is "linear", i.e., it lacks the AND-OR alternation aspect of $\mathcal{G} \mathcal{A} \mathcal{L} \mathcal{T}$. After execution of a reachable transition $t \stackrel{\text { a }}{\rightarrow} s$ of $\left(\mathrm{B}, Q_{0}\right)$, we consider the in-depth and out-depth of the components $\mathrm{B}_{i} \in$ components(a). Suppose that there exists a supercycle $S C \sqsubseteq W_{\mathrm{B}}(s)$. There are three cases:

- Case 1. $\mathrm{B}_{i}$ has finite in-depth in $W_{\mathrm{B}}(s)$ : then, if $\mathrm{B}_{i} \in S C$, it can be removed and still leave a supercycle $S C^{\prime}$, by Proposition 4.27. Hence $S C^{\prime} \sqsubseteq W_{\mathrm{B}}(t)$, and so $\mathrm{B}_{i}$ is not essential to the creation of a supercycle. 
- Case 2. $\mathrm{B}_{i}$ has finite out-depth in $W_{\mathrm{B}}(s)$ : by Corollary $4.25, \mathrm{~B}_{i}$ cannot be part of a supercycle, and so $S C \sqsubseteq W_{\mathrm{B}}(t)$.

- Case 3. $\mathrm{B}_{i}$ has infinite in-depth and infinite out-depth in $W_{\mathrm{B}}(s)$ : in this case, $\mathrm{B}_{i}$ is possibly an essential part of $S C$, i.e., $S C$ was created in going from $t$ to $s$.

We thus impose a condition which guarantees that only Case 1 or Case 2 occur.

Definition $5.6\left(\mathcal{G} \mathcal{L} \mathcal{I} \mathcal{N}\left(\mathrm{B}, Q_{0}\right.\right.$, a) ). Let $t \stackrel{\text { a }}{\rightarrow} s$ be a reachable transition of $\left(\mathrm{B}, Q_{0}\right)$. Then, in $W_{\mathrm{B}}(s)$, every component $\mathrm{B}_{i}$ of components $(\mathrm{a})$ either has finite in-depth, or has finite out-depth. Formally,

$\forall \mathrm{B}_{i} \in$ components $(a):$ in_depth $\mathrm{B}_{\mathrm{B}}\left(\mathrm{B}_{i}, s\right)<\omega \vee$ out_depth $\mathrm{B}_{\mathrm{B}}\left(\mathrm{B}_{i}, s\right)<\omega$.

Proposition 5.7. Assume that node $v$ of $W_{\mathrm{B}}(s)$ has a finite in-depth of $d$ in $W_{\mathrm{B}}(s)$, i.e., in_depth $h_{\mathrm{B}}(v, s)=d$. Then $\mathrm{sConnViol}_{\mathrm{B}}(v, s)$.

Proof. A node with finite in-depth cannot be in a wait-for cycle (i.e., a cycle of wait-for edges), and therefore cannot be in a strongly connected supercycle.

Lemma 5.8. For all interactions $\mathrm{a} \in \gamma: \mathcal{G} \mathcal{L} \mathcal{I} \mathcal{N}\left(\mathrm{B}, Q_{0}\right.$, a) implies $\mathcal{G} \mathcal{A} \mathcal{L} \mathcal{T}\left(\mathrm{B}, Q_{0}, \mathrm{a}\right)$.

Proof. Assume, for arbitrary a $\in \gamma$, that $\operatorname{GLIN}\left(\mathrm{B}, Q_{0}, \mathrm{a}\right)$ holds. That is,

For every reachable transition $t \stackrel{\mathrm{a}}{\rightarrow} s$ of $\left(\mathrm{B}, Q_{0}\right)$,

$\forall \mathrm{B}_{i} \in$ components $(\mathrm{a}):$ in_depth $\left(\mathrm{B}_{i}, s\right)<\omega \vee$ out_depth $\left(\mathrm{B}_{i}, s\right)<\omega$.

By Propositions 4.24 and 5.7,

For every reachable transition $t \stackrel{\mathrm{a}}{\rightarrow} s$ of $\left(\mathrm{B}, Q_{0}\right)$,

$\forall \mathrm{B}_{i} \in$ components $(\mathrm{a}): \mathrm{sConnViol}_{\mathrm{B}}\left(\mathrm{B}_{i}, s\right) \vee\left(\exists d \geq 1: \operatorname{viol}_{\mathrm{B}}\left(\mathrm{B}_{i}, s, d\right)\right)$.

Hence by Proposition 4.14 and Definition 5.3,

For every reachable transition $t \stackrel{\mathrm{a}}{\rightarrow} s$ of $\left(\mathrm{B}, Q_{0}\right)$,

$\forall \mathrm{B}_{i} \in$ components(a) : genViol ${ }_{\mathrm{B}}\left(\mathrm{B}_{i}, s\right)$

Hence $\mathcal{G} \mathcal{A} \mathcal{L} \mathcal{T}\left(\mathrm{B}, Q_{0}\right.$, a) holds.

Theorem 5.9. $\mathcal{G} \mathcal{L} \mathcal{I}$ is supercycle-freedom preserving

Proof. Follows immediately from Theorem 5.5 and Lemma 5.8.

\subsection{Deadlock freedom using global restrictions}

Corollary 5.10 (Deadlock-Freedom via $\mathcal{G} \mathcal{A} \mathcal{L} \mathcal{T}, \mathcal{G} \mathcal{L} \mathcal{N}$ ). Assume that

(1) for all $s_{0} \in Q_{0}, W_{\mathrm{B}}\left(s_{0}\right)$ is supercycle-free, and

(2) for all interactions a of $\mathrm{B}($ i.e., $\mathrm{a} \in \gamma): \mathcal{G} \mathcal{A} \mathcal{L} \mathcal{T}\left(\mathrm{B}, Q_{0}, \mathrm{a}\right) \vee \mathcal{G} \mathcal{L} \mathcal{I} \mathcal{N}\left(\mathrm{B}, Q_{0}\right.$, a) holds.

Then for every reachable state $u$ of $\left(\mathrm{B}, Q_{0}\right): W_{\mathrm{B}}(u)$ is supercycle-free, and so $\left(\mathrm{B}, Q_{0}\right)$ is free of local deadlock.

Proof. Immediate from Corollary 3.14, Theorem 5.5, and Theorem 5.9.

\section{LOCAL SUPERCYCLES}

Evaluating the global restrictions $\mathcal{G} \mathcal{A} \mathcal{L} \mathcal{T}\left(\mathrm{B}, Q_{0}\right.$, a), $\mathcal{G} \mathcal{L} \mathcal{I} \mathcal{N}\left(\mathrm{B}, Q_{0}\right.$, a) requires checking all reachable transitions of $\left(\mathrm{B}, Q_{0}\right)$, which is, in general, subject to state-explosion. We need restrictions which imply $\mathcal{G} \mathcal{A} \mathcal{L} \mathcal{T}\left(\mathrm{B}, Q_{0}, \mathrm{a}\right), \mathcal{G} \mathcal{L} \mathcal{I} \mathcal{N}\left(\mathrm{B}, Q_{0}\right.$, a), and which can be checked efficiently. To this end, we first develop some terminology, and a projection result, for relating the waiting-behavior in a subsystem of $\left(\mathrm{B}, Q_{0}\right)$ to that in $\left(\mathrm{B}, Q_{0}\right)$ overall. 


\subsection{Projection onto subsystems}

Definition 6.1 (Projection of a wait-for graph). Let $\left(\mathrm{B}^{\prime}, Q_{0}^{\prime}\right)$ be a subsystem of $\left(\mathrm{B}, Q_{0}\right) . W_{\mathrm{B}}(s)\left\lceil\mathrm{B}^{\prime}\right.$ is the wait-for graph whose nodes are the components and interactions in $\mathrm{B}^{\prime}$, and whose edges are the induced edges from $W_{\mathrm{B}}(s)$, i.e., for nodes $v, w$ of $W_{\mathrm{B}}(s)\left\lceil\mathrm{B}^{\prime}, v \rightarrow w\right.$ is an edge in $W_{\mathrm{B}}(s)\left\lceil\mathrm{B}^{\prime}\right.$ iff $v \rightarrow w$ is an edge in $W_{\mathrm{B}}(s)$.

Write $W_{\mathrm{B}^{\prime}}(s)$ for $W_{\mathrm{B}}(s)\left\lceil\mathrm{B}^{\prime}\right.$. Also, if $s^{\prime}=s\left\lceil\mathrm{~B}^{\prime}\right.$, then define $W_{\mathrm{B}^{\prime}}\left(s^{\prime}\right) \triangleq W_{\mathrm{B}}(s)\left\lceil\mathrm{B}^{\prime}\right.$, since $W_{\mathrm{B}}(s)\left\lceil\mathrm{B}^{\prime}\right.$ depends only on the projection of state $s$ onto $\mathrm{B}^{\prime}$.

We now show that wait-for behavior in B "projects down" to any subcomponent $\mathrm{B}^{\prime}$, and that wait-for behavior in B' "projects up" to B.

Proposition 6.2 (WAit-For EDge PROJECTION). Let $\mathrm{B}^{\prime}$ be a subcomponent of $\mathrm{B}$. Let s be a state of $\mathrm{B}$, and $s^{\prime}=s \backslash \mathrm{B}^{\prime}$. Let $\mathrm{a}$ be an interaction of $\mathrm{B}^{\prime}$, and $\mathrm{B}_{i} \in$ components(a) be an atomic component of $\mathrm{B}^{\prime}$. Then (1) $\mathrm{a} \rightarrow \mathrm{B}_{i} \in W_{\mathrm{B}}(s)$ iff $\mathrm{a} \rightarrow \mathrm{B}_{i} \in W_{\mathrm{B}^{\prime}}\left(s^{\prime}\right)$, and (2) $\mathrm{B}_{i} \rightarrow \mathrm{a} \in W_{\mathrm{B}}(s)$ iff $\mathrm{B}_{i} \rightarrow \mathrm{a} \in W_{\mathrm{B}^{\prime}}\left(s^{\prime}\right)$.

Proof. By Definition 3.3, a $\rightarrow \mathrm{B}_{i} \in W_{\mathrm{B}}(s)$ iff $s\left\lceil\mathrm{~B}_{i}\left(e n b_{\mathrm{a}}^{\mathrm{B}_{i}}\right)=\right.$ false. Since $s^{\prime}=s\left\lceil\mathrm{~B}^{\prime}\right.$, we have $s^{\prime} \backslash \mathrm{B}_{i}=s \backslash \mathrm{B}_{i}$. Hence $s \backslash \mathrm{B}_{i}\left(e n b_{\mathrm{a}}^{\mathrm{B}_{i}}\right)=s^{\prime} \backslash \mathrm{B}_{i}\left(e n b_{\mathrm{a}}^{\mathrm{B}_{i}}\right)$. By Definition 3.3, $a \rightarrow \mathrm{B}_{i} \in W_{\mathrm{B}^{\prime}}\left(s^{\prime}\right)$ iff $s^{\prime}\left\lceil\mathrm{B}_{i}\left(e n b_{\mathrm{a}}^{\mathrm{B}_{i}}\right)=\right.$ false. Putting together these three equalities gives us Clause (1).

By Definition 3.3, $\mathrm{B}_{i} \rightarrow \mathrm{a} \in W_{\mathrm{B}}(s)$ iff $s\left\lceil\mathrm{~B}_{i}\left(e n b_{\mathrm{a}}^{\mathrm{B}_{i}}\right)=\right.$ true. Since $s^{\prime}=s\left\lceil\mathrm{~B}^{\prime}\right.$, we have $s^{\prime}\left\lceil\mathrm{B}_{i}=s\left\lceil\mathrm{~B}_{i}\right.\right.$. Hence $s \backslash \mathrm{B}_{i}\left(e n b_{\mathrm{a}}^{\mathrm{B}_{i}}\right)=s^{\prime} \backslash \mathrm{B}_{i}\left(e n b_{\mathrm{a}}^{\mathrm{B}_{i}}\right)$. By Definition 3.3, $\mathrm{B}_{i} \rightarrow \mathrm{a} \in W_{\mathrm{B}^{\prime}}\left(s^{\prime}\right)$ iff $s^{\prime} \backslash \mathrm{B}_{i}\left(e n b_{\mathrm{a}}^{\mathrm{B}_{i}}\right)=$ true . Putting the above three equalities together gives us clause (2).

Definition 6.3 (Structure graph $G_{\mathrm{B}}, G_{\mathrm{a}}^{\ell}$ ). The structure graph $G_{\mathrm{B}}$ of composite component $\mathrm{B}=$ $\gamma\left(B_{1}, \ldots, B_{n}\right)$ is a bipartite graph whose nodes are the $B_{1}, \ldots, B_{n}$ and all the $a \in \gamma$. There is an edge between $\mathrm{B}_{i}$ and interaction a iff $\mathrm{B}_{i}$ participates in a, i.e., $\mathrm{B}_{i} \in$ components(a). Define the distance between two nodes to be the number of edges in a shortest path between them. Let $G_{\mathrm{a}}^{\ell}$ be the subgraph of $G_{\mathrm{B}}$ that contains a and all nodes of $G_{\mathrm{B}}$ that have a distance to a which is less than or equal to $\ell$.

Definition 6.4 (Deadlock-checking subsystem, $\mathrm{D}_{\mathrm{a}}^{\ell}$ ). Define $\mathrm{D}_{\mathrm{a}}^{\ell}$, the deadlock-checking subsystem for interaction a and radius $\ell$, to be the subsystem of $\left(\mathrm{B}, Q_{0}\right)$ based on the set of components in $G_{\mathrm{a}}^{2 \ell}$. (See Definition 2.12).

Definition 6.5 (Border node, interior node of $\mathrm{D}_{\mathrm{a}}^{\ell}$ ). A node $v$ of $\mathrm{D}_{\mathrm{a}}^{\ell}$ is a border-node iff it has an edge in $G_{\mathrm{B}}$ to a node outside of $\mathrm{D}_{\mathrm{a}}^{\ell}$. If node $v$ of $\mathrm{D}$ is not a border node, then it is an internal node.

Note that all border nodes of $\mathrm{D}_{\mathrm{a}}^{\ell}$ are interactions, since $2 \ell$ is even. Hence all component nodes of $\mathrm{D}_{\mathrm{a}}^{\ell}$ are interior nodes.

In the sequel, we fix a particular subsystem $\mathrm{D}_{\mathrm{a}}^{\ell}$, which we refer to simply as $\mathrm{D}$, with a and $\ell$ being implicit (to avoid notational clutter with double-sub/superscripts). We write D.action = a and D.radius $=\ell$. Also, let $Q_{0}^{\mathrm{D}}=Q_{0}\left\lceil\mathrm{D}\right.$, i.e., $Q_{0}^{\mathrm{D}}$ is the set of initial states of $\mathrm{D}$, and let $s_{\mathrm{D}}$ be an arbitrary state of $\mathrm{D}$. As given above, for a state $s_{\mathrm{D}}$ of $\mathrm{D}$, the wait-for graph for $\mathrm{D}$ only (i.e., ignoring the components and interactions of $B$ that are not in $D)$ is denoted as $W_{\mathrm{D}}\left(s_{\mathrm{D}}\right)$. Note also that "the nodes of $\mathrm{D}$ " and "the nodes of $W_{\mathrm{D}}\left(s_{\mathrm{D}}\right)$ " denote the same set of components and interactions. We will use either expression, depending on context.

\subsection{Fixpoint characterization of local supercycles in a subsystem}

We now develop a local version of the sequence of definitions and propositions given in Section 4.1. Local means that they apply to any subsystem $\left(\mathrm{B}^{\prime}, Q_{0}^{\prime}\right)$ of $\left(\mathrm{B}, Q_{0}\right)$. A subsystem has, in general, border nodes, i.e., those nodes with a neighbor outside of the subsystem. The supercycle membership of these nodes cannot be determined with certainty, by inspecting just the subsystem. Hence we 
pessimistically assume that border nodes are in a supercycle. When false, this assumption may produce a false negative, and so we sacrifice completeness of our deadlock-freedom criterion. We do however, avoid false positives (that may result if we assume a border node is not in a supercycle when in fact it is), and so we maintain soundness of our criterion.

Definition 6.6 (Local supercycle). Let $\mathrm{B}=\gamma\left(\mathrm{B}_{1}, \ldots, \mathrm{B}_{n}\right)$ be a composite component, $\mathrm{D}$ a subsystem of $\mathrm{B}$, and $s_{\mathrm{D}}$ a state of $\mathrm{D}$. A subgraph $S C$ of $W_{\mathrm{D}}\left(s_{\mathrm{D}}\right)$ is a local supercycle in $W_{\mathrm{D}}\left(s_{\mathrm{D}}\right)$ if and only if all of the following hold:

1. $S C$ is nonempty, i.e., contains at least one node,

2. if $\mathrm{B}_{i}$ is a node in $S C$, then for all interactions a such that there is an edge in $W_{\mathrm{D}}\left(s_{\mathrm{D}}\right)$ from $\mathrm{B}_{i}$ to a:

(a) a is a node in $S C$, and

(b) there is an edge in $S C$ from $B_{i}$ to a,

that is, $\mathrm{B}_{i} \rightarrow \mathrm{a} \in W_{\mathrm{D}}\left(s_{\mathrm{D}}\right)$ implies $\mathrm{B}_{i} \rightarrow \mathrm{a} \in S C$,

3. if a is a node in $S C$, then, either a is a border interaction of $\mathrm{D}$, or there exists a $\mathrm{B}_{j}$ such that:

(a) $\mathrm{B}_{j}$ is a node in $S C$, and

(b) there is an edge from a to $\mathrm{B}_{j}$ in $W_{\mathrm{D}}\left(s_{\mathrm{D}}\right)$, and

(c) there is an edge from a to $\mathrm{B}_{j}$ in $S C$,

that is, a $\in S C$ implies $\left(\exists \mathrm{B}_{j}: \mathrm{a} \rightarrow \mathrm{B}_{j} \in W_{\mathrm{D}}\left(s_{\mathrm{D}}\right) \wedge \mathrm{a} \rightarrow \mathrm{B}_{j} \in S C\right)$ or (a is a border interaction of D).

Intuitively, $S C$ is a supercycle iff every node in $S C$ is blocked from executing by other nodes in $S C$, or is a border interaction. We pessimistically consider a border interaction a to be blocked, since the subsystem $\mathrm{D}$ cannot provide information about the participant components of a that are outside of D. In particular, one or more border interactions necessarily form a local supercycle. Yet, it is important to notice that a blocked border interaction a does not necessarily imply a global supercycle.

We carry over the definition of subgraph $\sqsubseteq$ from Section 4.1, and develop the analogous definitions for the subsystem D of B.

Definition 6.7 (Set of subgraphs). $\mathcal{P}\left(W_{\mathrm{D}}\left(s_{\mathrm{D}}\right)\right) \triangleq\left\{X \mid X \sqsubseteq W_{\mathrm{D}}\left(s_{\mathrm{D}}\right)\right\}$.

Definition 6.8 (Wait-for lattice). Define the partially ordered set $\mathcal{L}_{\mathrm{D}}\left(s_{\mathrm{D}}\right)=\left\langle\mathcal{P}\left(W_{\mathrm{D}}\left(s_{\mathrm{D}}\right)\right)\right.$, $一$ whose elements are all the subgraphs of $W_{\mathrm{D}}\left(s_{\mathrm{D}}\right)$, and where $U \sqsubseteq V$ is as in Definition 3.4.

Proposition 6.9. $\mathcal{L}_{\mathrm{D}}\left(s_{\mathrm{D}}\right)=\left\langle\mathcal{P}\left(W_{\mathrm{D}}\left(s_{\mathrm{D}}\right)\right)\right.$, ᄃ $\rangle$ is a finite complete Boolean lattice, with $\sqcap, \sqcup$, and complementation as in Proposition 4.3, top element $W_{\mathrm{D}}\left(s_{\mathrm{D}}\right)$, and bottom element $\emptyset$.

Definition $6.10\left(\right.$ lblocks $\left._{s_{\mathrm{D}}}\right)$. Let $X \sqsubseteq W_{\mathrm{D}}\left(s_{\mathrm{D}}\right)$ and a, $\mathrm{B}_{i}$ be nodes in $W_{\mathrm{D}}\left(s_{\mathrm{D}}\right)$. Then lblocks $_{s_{\mathrm{D}}}(\mathrm{a}, X) \triangleq$ $\left[\left(\exists \mathrm{B}_{i} \in X: \mathrm{a} \rightarrow \mathrm{B}_{i} \in W_{\mathrm{D}}\left(s_{\mathrm{D}}\right)\right)\right.$ or a is a border interaction of $\left.\mathrm{D}\right]$, and $\operatorname{lblocks}_{s_{\mathrm{D}}}\left(\mathrm{B}_{i}, X\right) \triangleq(\forall \mathrm{a}:$ $\left.\mathrm{B}_{i} \rightarrow \mathrm{a} \in W_{\mathrm{D}}\left(s_{\mathrm{D}}\right) \Rightarrow \mathrm{a} \in X\right)$.

Hence a border interaction a is pessimistically considered to be always blocked, since the subsystem $D$ does not contain enough information about the enablement of a. A non-border interaction a is (as usual) blocked by a set of nodes $X$ if some participant $\mathrm{B}_{i}$ of a is in $X$, and $\mathrm{B}_{i}$ does not enable a. A component $\mathrm{B}_{i}$ is blocked by $X$ if all of the interactions that $\mathrm{B}_{i}$ enables are in $X$.

Definition $6.11\left(\mathcal{S} \mathcal{L}_{s_{\mathrm{D}}}\right)$. Define $\mathcal{S} \mathcal{L}_{s_{\mathrm{D}}}: \mathcal{P}\left(W_{\mathrm{D}}\left(s_{\mathrm{D}}\right)\right) \rightarrow \mathcal{P}\left(W_{\mathrm{D}}\left(s_{\mathrm{D}}\right)\right)$ as follows. $\mathcal{S} \mathcal{L}_{s_{\mathrm{D}}}(X)$ is the subgraph with nodes $\left\{v \mid\right.$ lblocks $\left._{s_{\mathrm{D}}}(v, X)\right\}$, together with the edges induced by $W_{\mathrm{D}}\left(s_{\mathrm{D}}\right)$.

Definition $6.12\left(\mathcal{V} \mathcal{L}_{s_{\mathrm{D}}}\right)$. Define $\mathcal{V} \mathcal{L}_{s_{\mathrm{D}}}: \mathcal{P}\left(W_{\mathrm{D}}\left(s_{\mathrm{D}}\right)\right) \rightarrow \mathcal{P}\left(W_{\mathrm{D}}\left(s_{\mathrm{D}}\right)\right)$ as follows. $\mathcal{V} \mathcal{L}_{s_{\mathrm{D}}}(X)$ is the subgraph with nodes $\left\{v \mid \neg\right.$ lblocks $\left._{s_{\mathrm{D}}}(v, \widehat{X})\right\}$, together with the edges induced by $W_{\mathrm{D}}\left(s_{\mathrm{D}}\right)$, where we take the complement $\widehat{X}$ with respect to D. 
Implicit in writing $\widehat{X}$ is that $X \sqsubseteq \mathrm{D}$. Then $\widehat{X}$ contains all nodes that are in $\mathrm{D}$ and not in $X$, together with the edges induced by $W_{\mathrm{D}}\left(s_{\mathrm{D}}\right)$. Hence $\left.\mathcal{V} \mathcal{L}_{s_{\mathrm{D}}}(X)=\widehat{\mathcal{S} \mathcal{L}_{s_{\mathrm{D}}}(\widehat{X}}\right)$, i.e., $\mathcal{V} \mathcal{L}_{s_{\mathrm{D}}}$ and $\mathcal{S} \mathcal{L}_{s_{\mathrm{D}}}$ are duals. Note that (as for $\mathcal{S}_{s}$ and $\left.\mathcal{V}_{s}\right) \mathcal{S} \mathcal{L}_{s_{\mathrm{D}}}$ and $\mathcal{V} \mathcal{L}_{s_{\mathrm{D}}}$ are defined given a particular subsystem $\mathrm{D}$ of the system $\mathrm{B}$, and a particular state $s_{\mathrm{D}}$ of $\mathrm{D}$. Since the subsystem $\mathrm{D}$ is fixed, we will omit $\mathrm{D}$ from the subscript of $\mathcal{S} \mathcal{L}_{s_{\mathrm{D}}}$ and $\mathcal{V} \mathcal{L}_{S_{\mathrm{D}}}$.

Proposition 6.13. $\mathcal{S} \mathcal{L}_{s_{\mathrm{D}}}$ and $\mathcal{V} \mathcal{L}_{s_{\mathrm{D}}}$ are monotone and continuous.

Proof. We show first that $\mathcal{S} \mathcal{L}_{s_{\mathrm{D}}}$ is monotone, i.e., $X \sqsubseteq Y \Rightarrow \mathcal{S} \mathcal{L}_{s_{\mathrm{D}}}(X) \sqsubseteq \mathcal{S} \mathcal{L}_{s_{\mathrm{D}}}(Y)$. Let $v$ be an arbitrary node in $\mathcal{S} \mathcal{L}_{s_{\mathrm{D}}}(X)$, so that lblocks $_{s_{\mathrm{D}}}(v, X)$ holds. There are three cases.

Case of $v$ is a border interaction of D. Then $\operatorname{lblocks}_{s_{\mathrm{D}}}(v, Y)$ by Definition 6.10, and so $v \in \mathcal{S} \mathcal{L}_{s_{\mathrm{D}}}(Y)$ by Definition 6.11.

Case of $v$ is a non-border interaction a. By Definitions 6.10 and 6.11, we have $\exists \mathrm{B}_{i} \in X: \mathrm{a} \rightarrow \mathrm{B}_{i} \in W_{\mathrm{D}}\left(s_{\mathrm{D}}\right)$. Since $X \sqsubseteq Y$, this same $\mathrm{B}_{i}$ is also a node of $Y$, and so $\exists \mathrm{B}_{i} \in Y: \mathrm{a} \rightarrow \mathrm{B}_{i} \in W_{\mathrm{D}}\left(s_{\mathrm{D}}\right)$. Hence lblocks $_{s_{\mathrm{D}}}(\mathrm{a}, Y)$, and so a $\in \mathcal{S} \mathcal{L}_{s_{\mathrm{D}}}(Y)$.

Case of $v$ is a component $\mathrm{B}_{i}$. By Definitions 6.10 and 6.11, we have $\left(\forall \mathrm{a}: \mathrm{B}_{i} \rightarrow \mathrm{a} \in W_{\mathrm{D}}\left(s_{\mathrm{D}}\right) \Rightarrow\right.$ $\mathrm{a} \in X)$. Since $X \sqsubseteq Y$, we have $\left(\forall \mathrm{a}: \mathrm{B}_{i} \rightarrow \mathrm{a} \in W_{\mathrm{D}}\left(s_{\mathrm{D}}\right) \Rightarrow \mathrm{a} \in Y\right)$. Hence, lblocks $s_{s_{\mathrm{D}}}\left(\mathrm{B}_{i}, Y\right)$, and so $\mathrm{B}_{i} \in \mathcal{S} \mathcal{L}_{S_{\mathrm{D}}}(Y)$.

In all three cases, we have $v \in \mathcal{S} \mathcal{L}_{S_{\mathrm{D}}}(Y)$. Since $v$ was chosen arbitrarily from $\mathcal{S} \mathcal{L}_{S_{\mathrm{D}}}(X)$, it follows that $\mathcal{S} \mathcal{L}_{s_{\mathrm{D}}}(X) \sqsubseteq \mathcal{S} \mathcal{L}_{s_{\mathrm{D}}}(Y)$. Hence, $\mathcal{S} \mathcal{L}_{s_{\mathrm{D}}}$ is monotone. Since the dual of a monotone mapping in a complete Boolean lattice is also monotone, we have that $\mathcal{V} \mathcal{L}_{s_{\mathrm{D}}}$ is monotone. Finally, since $\mathcal{L}_{\mathrm{D}}\left(s_{\mathrm{D}}\right)$ is finite, it follows that $\mathcal{S} \mathcal{L}_{s_{\mathrm{D}}}$ and $\mathcal{V} \mathcal{L}_{s_{\mathrm{D}}}$ are continuous.

Proposition 6.14. Let $X \neq \emptyset$ and $X \sqsubseteq W_{\mathrm{D}}\left(s_{\mathrm{D}}\right)$, i.e., $X$ is a non-empty subgraph of $W_{\mathrm{D}}\left(s_{\mathrm{D}}\right)$. Then, $X$ is a local supercycle in $W_{\mathrm{D}}\left(s_{\mathrm{D}}\right)$ iff $X \sqsubseteq \mathcal{S} \mathcal{L}_{s_{\mathrm{D}}}(X)$.

Proof. Let $X$ be a local supercycle in $W_{\mathrm{D}}\left(s_{\mathrm{D}}\right)$. By Definition 6.6, every node in $X$ is blocked by $X$ or is a border interaction, i.e., $\left(\forall x \in X:\right.$ lblocks $\left._{s_{\mathrm{D}}}(x, X)\right)$. By Definition 4.5, $X \sqsubseteq \mathcal{S} \mathcal{L}_{s_{\mathrm{D}}}(X)$.

Conversely, suppose $X \sqsubseteq \mathcal{S} \mathcal{L}_{s_{\mathrm{D}}}(X)$ for some subgraph $X$ of $W_{\mathrm{D}}\left(s_{\mathrm{D}}\right)$. Hence $(\forall x \in X: x \in$ $\left.\mathcal{S} \mathcal{L}_{s_{\mathrm{D}}}(X)\right)$, so by Definition 6.11, $\left(\forall x \in X:\right.$ lblocks $\left._{s_{\mathrm{D}}}(x, X)\right)$. Hence every node in $X$ is blocked by $X$ or is a border interaction, and so $X$ satisfies Definition 6.6, and is therefore a local supercycle.

PRoposition 6.15. Let $S C, S C^{\prime}$ be local supercycles in $W_{\mathrm{D}}\left(s_{\mathrm{D}}\right)$. Then $S C \sqcup S C^{\prime}$ is a local supercycle in $W_{\mathrm{D}}\left(s_{\mathrm{D}}\right)$.

Proof. By Proposition 6.14, $S C$ and $S C^{\prime}$ are post-fixpoints of $\mathcal{S} \mathcal{L}_{S_{\mathrm{D}}}$. Since the join of postfixpoints is a post-fixpoint, the proposition follows by applying Proposition 6.14 again.

Proposition 6.16. Let $S C$ be the greatest fixpoint of $\mathcal{S} \mathcal{L}_{s_{\mathrm{D}}}$. Then either $(a) W_{\mathrm{D}}\left(s_{\mathrm{D}}\right)$ is supercyclefree and $S C=\emptyset$, or $(b) W_{\mathrm{D}}\left(s_{\mathrm{D}}\right)$ contains local supercycles, and $S C$ is the largest local supercycle in $W_{\mathrm{D}}\left(s_{\mathrm{D}}\right)$.

Proof. By the Knaster-Tarski theorem, the greatest fixpoint is the join of all the post-fixpoints. If $W_{\mathrm{D}}\left(s_{\mathrm{D}}\right)$ is supercycle-free, then by Proposition 6.14, the only post-fixpoint of $\mathcal{S} \mathcal{L}_{s_{\mathrm{D}}}$ is $\emptyset$. Hence $S C=\emptyset$ (this is possible since there may be no border interactions). If $W_{\mathrm{D}}\left(s_{\mathrm{D}}\right)$ contains supercycles, then by Proposition 6.14, the set of post-fixpoints of $\mathcal{S} \mathcal{L}_{s_{\mathrm{D}}}$ is exactly the set of local supercycles of $W_{\mathrm{D}}\left(s_{\mathrm{D}}\right)$. Hence $S C$ is the join of all these local supercycles. By Proposition 6.15, $S C$ is the largest local supercycle in $W_{\mathrm{D}}\left(s_{\mathrm{D}}\right)$. 
Proposition 6.17. $v \in l f p\left(\mathcal{V} \mathcal{L}_{s_{\mathrm{D}}}\right)$ iff $v$ is not a node in any local supercycle of $W_{\mathrm{D}}\left(s_{\mathrm{D}}\right)$.

Proof. From the Park conjugate (dual) fixpoint theorem in complete Boolean lattices [27], we have $l f p\left(\mathcal{V}_{s_{\mathrm{D}}}\right)=\overline{g f p\left(\mathcal{S}_{S_{\mathrm{D}}}\right)}$. By Proposition 4.10, $g f p\left(\mathcal{S}_{S_{\mathrm{D}}}\right)$ is the largest local supercycle in $W_{\mathrm{D}}\left(s_{\mathrm{D}}\right)$. Hence the nodes not in $g f p\left(\mathcal{S}_{S_{\mathrm{D}}}\right)$ are exactly the nodes that are not in any local supercycle. These are exactly the nodes in $l f p\left(\mathcal{V}_{S_{\mathrm{D}}}\right)$.

Define $\mathcal{V} \mathcal{L}_{s_{\mathrm{D}}}^{1}(X)=\mathcal{V} \mathcal{L}_{s_{\mathrm{D}}}(X)$, and for $d>1, \mathcal{V} \mathcal{L}_{s_{\mathrm{D}}}^{d}(X)=\mathcal{V} \mathcal{L}_{s_{\mathrm{D}}}\left(\mathcal{V} \mathcal{L}_{s_{\mathrm{D}}}^{d-1}(X)\right)$, i.e., a superscript indicates functional iteration of $\mathcal{V} \mathcal{L}_{s_{\mathrm{D}}}$. Note that $\mathcal{V} \mathcal{L}_{s_{\mathrm{D}}}^{d}(\emptyset) \sqsubseteq \mathcal{V} \mathcal{L}_{s_{\mathrm{D}}}^{d^{\prime}}(\emptyset)$ when $d \leq d^{\prime}$, since $\mathcal{V} \mathcal{L}_{s_{\mathrm{D}}}$ is monotone. Hence $\mathcal{V} \mathcal{L}_{s_{\mathrm{D}}}^{1}(\emptyset), \mathcal{V} \mathcal{L}_{s_{\mathrm{D}}}^{2}(\emptyset), \ldots$ is a non-decreasing sequence.

Proposition 6.18. $\operatorname{lfp}\left(\mathcal{V} \mathcal{L}_{s_{\mathrm{D}}}\right)=\bigsqcup_{d \geq 1} \mathcal{V} \mathcal{L}_{s_{\mathrm{D}}}^{d}(\emptyset)$

Proof. $\mathcal{V} \mathcal{L}_{s_{\mathrm{D}}}$ is continuous. Follows by standard results, e.g., see the $\mathrm{CPO}$ fixpoint theorem I in [20].

Definition 6.19 (Local supercycle violation, $\operatorname{viol}_{\operatorname{Loc}}\left(v, s_{\mathrm{D}}\right)$, $\operatorname{violLoc}_{\mathrm{D}}\left(v, s_{\mathrm{D}}, d\right)$ ). Let $s_{\mathrm{D}}$ be a state of $\mathrm{D}$ and $v$ be a node of $\mathrm{D}$. Define $\operatorname{viol}_{\operatorname{Loc}_{\mathrm{D}}}\left(v, s_{\mathrm{D}}\right) \triangleq v \in \operatorname{lfp}\left(\mathcal{V} \mathcal{L}_{s_{\mathrm{D}}}\right)$, and, for $d \geq 1$, $\operatorname{violLoc}_{\mathrm{D}}\left(v, s_{\mathrm{D}}, d\right) \triangleq v \in \mathcal{V} \mathcal{L}_{s_{\mathrm{D}}}^{d}(\emptyset)$.

Proposition 6.20. $\operatorname{violLoc}_{\mathrm{D}}\left(v, s_{\mathrm{D}}\right)$ iff $\left(\exists d \geq 1: \operatorname{violLoc}_{\mathrm{D}}\left(v, s_{\mathrm{D}}, d\right)\right)$.

Proof. By Definition 6.19, violLoc $\left(v, s_{\mathrm{D}}\right) \equiv v \in \operatorname{lfp}\left(\mathcal{V} \mathcal{L}_{s_{\mathrm{D}}}\right)$. By Proposition 6.18, $v \in$ lfp $\left(\mathcal{V} \mathcal{L}_{s_{\mathrm{D}}}\right) \equiv v \in \bigsqcup_{d \geq 1} \mathcal{V} \mathcal{L}_{s_{\mathrm{D}}}^{d}(\emptyset)$. By Definition 6.19, $\left(\forall d \geq 1: \operatorname{violLoc}_{\mathrm{D}}\left(v, s_{\mathrm{D}}, d\right) \equiv v \in\right.$ $\left.\mathcal{V} \mathcal{L}_{s_{\mathrm{D}}}^{d}(\emptyset)\right)$. Chaining these equivalences establishes the proposition.

$\operatorname{violLoc}_{\mathrm{D}}\left(v, s_{\mathrm{D}}, d\right)$ defines a local supercycle violation that can be confirmed within $d$ iterations of $\mathcal{V} \mathcal{L}_{s_{\mathrm{D}}}$, which we call a level-d local supercycle violation. violLoc ${ }_{\mathrm{D}}\left(v, s_{\mathrm{D}}\right)$ requires, in general, the entire least fixpoint of $\mathcal{V} \mathcal{L}_{s_{\mathrm{D}}}$.

Example 6.21 (Local supercycle violations in dining philosophers). Figures 8(a), 8(b), and 8(c) illustrate local supercycle violations corresponding to Figures 7(b), 7(c), 7(d) respectively. The subsystem used, in each case, is based on the last interaction executed, i.e., $\mathrm{Grab}_{0}, \mathrm{Grab}_{2}$, and $\operatorname{Rel}_{0}$, respectively, and with a radius of 1 in all cases. The border interactions are shown underlined, and for each node $v$ (interaction or component), we include a small positive integer after its name, giving the smallest $d$ such that $v \in \mathcal{V} \mathcal{L}^{d}(\emptyset)$, i.e., the local supercycle violation level.

We now show that a local supercycle violation implies (global) supercycle violation.

Proposition 6.22. Let $s$ be an arbitrary global state of $\mathrm{B}$, and let $\left.s_{\mathrm{D}}=s\right\rceil \mathrm{D}$.

(a) Let $X \sqsubseteq W_{\mathrm{D}}\left(s_{\mathrm{D}}\right)$. Then $\mathcal{V} \mathcal{L}_{s_{\mathrm{D}}}(X) \sqsubseteq \mathcal{V}_{s}(X)$.

(b) Let $d \geq 1$. Then $\mathcal{V} \mathcal{L}_{s_{\mathrm{D}}}^{d}(\emptyset) \sqsubseteq \mathcal{V}_{s}^{d}(\emptyset)$.

Proof. For (a), let $v \in \mathcal{V} \mathcal{L}_{s_{\mathrm{D}}}(X)$. By Definition 6.12, $\neg$ lblocks $s_{s_{\mathrm{D}}}(v, \widehat{X})$. Now $v$ is either an interaction a or a component $\mathrm{B}_{i}$.

By Definition 6.10, if $v$ is an interaction a, then it is not a border interaction, and furthermore there is no component $\mathrm{B}_{i} \in \widehat{X}$ such that $\mathrm{a} \rightarrow \mathrm{B}_{i} \in W_{\mathrm{D}}\left(s_{\mathrm{D}}\right)$. Since $\widehat{X} \sqsubseteq \bar{X}$, we conclude $\neg$ blocks $(v, \bar{X})$, and so $v \in \mathcal{V}_{s}(X)$.

By Definition 6.10, if $v$ is a component $\mathrm{B}_{i}$, then there exists an interaction a such that $\mathrm{B}_{i} \rightarrow \mathrm{a} \in$ $W_{\mathrm{D}}\left(s_{\mathrm{D}}\right)$ and $\mathrm{a} \notin \widehat{X}$. Hence $\mathrm{a} \in X$, and so a $\notin \bar{X}$. Hence $\neg$ blocks $s(v, \bar{X})$, and so $v \in \mathcal{V}_{s}(X)$.

In both cases, the arbitrary element $v$ of $\mathcal{V} \mathcal{L}_{s_{\mathrm{D}}}(X)$ is also an element of $\mathcal{V}_{s}(X)$, and so $\mathcal{V} \mathcal{L}_{s_{\mathrm{D}}}(X) \sqsubseteq$ $\mathcal{V}_{s}(X)$. 


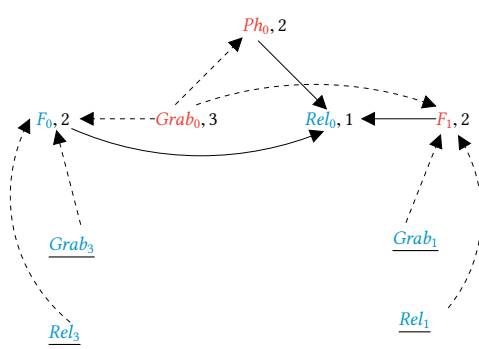

(a) Local supercycle violations after execution of $\mathrm{Grab}_{0}$.

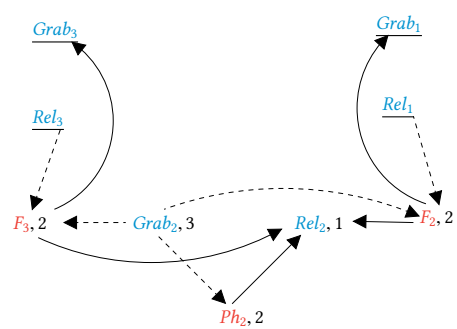

(b) Local supercycle violations after execution of $\mathrm{Grab}_{0} ; \mathrm{Grab}_{2}$.

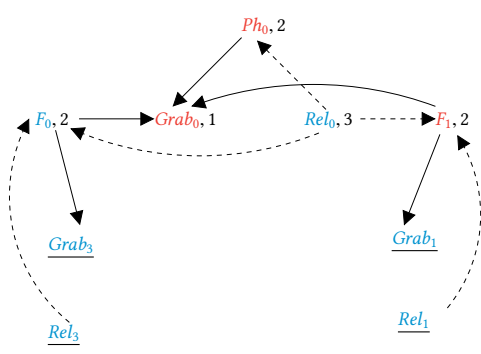

(c) Local supercycle violations after execution of $\mathrm{Grab}_{0} ; \mathrm{Grab}_{2} ; \mathrm{Rel}_{0}$.

Fig. 8. Example local supercycle violations for dining philosophers system of Figure 1.

We establish (b) by induction on $d$. The base case is $d=1$, which is given by (a). For the induction

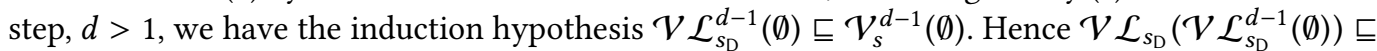
$\mathcal{V} \mathcal{L}_{s_{\mathrm{D}}}\left(\mathcal{V}_{s}^{d-1}(\emptyset)\right)$ since $\mathcal{V} \mathcal{L}_{s_{\mathrm{D}}}$ is monotone. By (a) $\mathcal{V} \mathcal{L}_{s_{\mathrm{D}}}\left(\mathcal{V}_{s}^{d-1}(\emptyset)\right) \sqsubseteq \mathcal{V}_{s}\left(\mathcal{V}_{s}^{d-1}(\emptyset)\right)$. Hence $\mathcal{V} \mathcal{L}_{s_{\mathrm{D}}}\left(\mathcal{V} \mathcal{L}_{s_{\mathrm{D}}}^{d-1}(\emptyset)\right) \sqsubseteq \mathcal{V}_{s}\left(\mathcal{V}_{s}^{d-1}(\emptyset)\right)$, i.e., $\mathcal{V} \mathcal{L}_{s_{\mathrm{D}}}^{d}(\emptyset) \sqsubseteq \mathcal{V}_{s}^{d}(\emptyset)$, and so (b) is established.

Proposition 6.23. Let $s$ be an arbitrary global state of $\mathrm{B}$. For all interactions $\mathrm{a} \in \gamma$ and $\ell \geq 1$, let $\mathrm{D}=\mathrm{D}_{\mathrm{a}}^{\ell}$ and $s_{\mathrm{D}}=s\lceil\mathrm{D}$. Then,

(a) For all $d \geq 1:$ violLoc $_{\mathrm{D}}\left(v, s_{\mathrm{D}}, d\right)$ implies viol $_{\mathrm{B}}(v, s, d)$, and

(b) $\operatorname{violLoc}_{\mathrm{D}}\left(v, s_{\mathrm{D}}\right)$ implies $\operatorname{viol}_{\mathrm{B}}(v, s)$.

Proof. For (a), assume violLoc $\left(v, s_{\mathrm{D}}, d\right)$ for some arbitrary $d \geq 1$. By Definition 6.19, $v \in$ $\mathcal{V} \mathcal{L}_{s_{\mathrm{D}}}^{d}(\emptyset)$. By Proposition 6.22, $v \in \mathcal{V}_{s}^{d}(\emptyset)$. By Definition 4.13, $\operatorname{viol}_{\mathrm{B}}(v, s, d)$.

For (b), assume violLoc $\mathrm{D}\left(v, s_{\mathrm{D}}\right)$. By Proposition 6.20, violLoc $\mathrm{D}\left(v, s_{\mathrm{D}}, d\right)$ for some $d \geq 1$. By (a), we have viol $\mathrm{B}(v, s, d)$. By Proposition 4.14, we have viol $\mathrm{B}(v, s)$.

\section{LOCAL CONDITIONS FOR DEADLOCK FREEDOM}

\subsection{A local AND-OR condition for deadlock-freedom}

We now seek a local condition, which we evaluate in $\mathrm{D}$, and which implies $\mathcal{G} \mathcal{A} \mathcal{L} \mathcal{T}$. We define local versions of both viol $\mathrm{B}(v, s, d)$ and $\mathrm{sConnViol}_{\mathrm{B}}(v, s)$.

To achieve a local and conservative approximation of $\operatorname{viol}_{\mathrm{B}}(v, s, d)$, we make the "pessimistic" assumption that the violation status of border nodes of $\mathrm{D}$ cannot be known, since it depends on nodes outside of D. Now, if an internal node $v$ of $\mathrm{D}$ can be marked with a level- $d$ local supercycleviolation, by applying Definition 6.19 to $\mathrm{D}$, and with the border nodes marked as non-violating, 
then it is also the case, as we show below, that $v$ also has a level- $d$ global supercycle-violation, as per Definition 4.13.

To achieve a local and conservative approximation of $\operatorname{sConnViol}_{\mathrm{B}}(v, s)$, we project onto the subsystem D.

7.1.1 Local strong connectedness condition. We now present the local version of the strong connectedness violation condition, given above in Definition 5.2.

Definition 7.1 (Local strong connectedness violation, $\mathrm{sConnViolLoc}_{\mathrm{D}}\left(v, s_{\mathrm{D}}\right)$ ). Let $L$ be the nodes of $W_{\mathrm{D}}\left(s_{\mathrm{D}}\right)$ that have no local supercycle violation, i.e., $L=\left\{v \mid v \in \mathrm{D} \wedge \neg \operatorname{viol} \operatorname{Loc}_{\mathrm{D}}\left(v, s_{\mathrm{D}}\right)\right\}$. Let $W L=W_{\mathrm{D}}\left(s_{\mathrm{D}}\right)\left\lceil L\right.$, i.e., $W L$ is the subgraph of $W_{\mathrm{D}}\left(s_{\mathrm{D}}\right)$ consisting of the nodes with no local supercycle violation, and the edges between those nodes that are also edges in $W_{\mathrm{D}}\left(s_{\mathrm{D}}\right)$.

Let $v$ be an arbitrary node in $W L$. Then, $\operatorname{sConnViolLoc}_{\mathrm{D}}\left(v, s_{\mathrm{D}}\right)$ holds iff:

(1) there does not exist a nontrivial strongly connected supercycle SSC such that $v \in S S C$ and $S S C \sqsubseteq W L$, and

(2) either

(a) there is no path in $W L$ from $v$ to a border node of $\mathrm{D}$ or

(b) there is no path in $W L$ from a border node of $\mathrm{D}$ to $v$.

Note that Clause 2a means that every wait-for path $\pi$ in $W_{\mathrm{D}}\left(s_{\mathrm{D}}\right)$ from $v$ to a border node of $\mathrm{D}$ contains at least one node $w$ with a local supercycle violation, i.e., violLoc $\operatorname{Li}_{\mathrm{D}}\left(w, s_{\mathrm{D}}\right)$. Also Clause $2 \mathrm{~b}$ means that every wait-for path $\pi^{\prime}$ in $W_{\mathrm{D}}\left(s_{\mathrm{D}}\right)$ from a border node of $\mathrm{D}$ to $v$ contains at least one node $w$ with a local supercycle violation, i.e., $\operatorname{viol}_{\operatorname{Loc}}\left(w, s_{\mathrm{D}}\right)$.

Table 1 summarizes the main predicates that we have defined. We show that the local strong connectedness condition implies the global strong connectedness condition.

Proposition 7.2. Let $s$ be an arbitrary state of $\mathrm{B}$. For all interactions $\mathrm{a} \in \gamma$, and $\ell \geq 1$, let $\mathrm{D}=\mathrm{D}_{\mathrm{a}}^{\ell}$, $s_{\mathrm{D}}=s \mid \mathrm{D}$, and let $v$ be an arbitrary node in $\mathrm{D}$. Then $\operatorname{sConnViolLoc}_{\mathrm{D}}\left(v, s_{\mathrm{D}}\right)$ implies $\mathrm{sConnViol} \mathrm{B}(v, s)$.

Proof. By contradiction. Assume for some state $s$ of $\mathrm{B}$ and some node $v$ in $\mathrm{D}$ that $\operatorname{sConnViolLoc}_{\mathrm{D}}\left(v, s_{\mathrm{D}}\right) \wedge \neg \mathrm{sConnViol} \mathrm{B}(v, s)$ holds. By $\neg \mathrm{sConnViol}_{\mathrm{B}}(v, s)$ and Definition 5.2 , there exists a strongly connected supercycle $S S C$ such that $v \in S S C$ and $S S C \sqsubseteq W_{\mathrm{B}}(s)$. Then, there are two cases:

(1) SSC $\sqsubseteq W_{\mathrm{D}}\left(s_{\mathrm{D}}\right)$ : let $x$ be any node in SSC. Since $x$ is a node in a supercycle, we have by Definition 4.17 and Proposition 4.18, that $\neg \mathrm{viol}_{\mathrm{B}}(x, s)$. Hence, by Proposition 6.23, we have $\neg \operatorname{viol}_{\text {Loc }}\left(x, s_{\mathrm{D}}\right)$. Let $W L$ be as given in Definition 7.1. Then $x \in W L$, and since $x$ is an arbitrary node of SSC, we have SSC $\sqsubseteq W L$. Thus Clause 1 of Definition 7.1 is violated.

(2) SSC $\nsubseteq W_{\mathrm{D}}\left(s_{\mathrm{D}}\right)$ : then there exists a node $x \in S S C-W_{\mathrm{D}}\left(s_{\mathrm{D}}\right)$. Now $v \in S S C$ and SSC is strongly connected. Hence there must exist a wait-for path $\pi$ in $W_{\mathrm{D}}\left(s_{\mathrm{D}}\right)$ from $v$ to $x$ and a wait-for path $\pi^{\prime}$ in $W_{\mathrm{D}}\left(s_{\mathrm{D}}\right)$ from $x$ to $v$. Since $v \in \mathrm{D}$ and $x \notin \mathrm{D}$, it follows that both $\pi$ and $\pi^{\prime}$ cross a border node of D. Furthermore, since $\pi, \pi^{\prime}$ are paths in SSC, every node $w$ that is in $\pi$ or in $\pi^{\prime}$ must be in a supercycle, and so cannot have a supercycle violation, i.e., $\neg v^{\prime o l o} l_{B}(w, s)$. By Proposition 6.23, every node $w$ that is in $\pi$ or in $\pi^{\prime}$ cannot have a local supercycle violation, i.e., $\neg$ viol $\operatorname{Loc}_{\mathrm{D}}\left(w, s_{\mathrm{D}}\right)$. Hence, Clauses $2 \mathrm{a}$ and $2 \mathrm{~b}$ of Definition 7.1 are violated, since they require that at least one node in $\pi$ and at least one node in $\pi^{\prime}$ has a local supercycle violation.

In both cases, Definition 7.1 is violated. But Definition 7.1 must hold, since we have $\mathrm{sConnViolLoc}_{\mathrm{D}}\left(v, s_{\mathrm{D}}\right)$. Hence, the desired contradiction. 
7.1.2 General local violation condition. We showed above that local supercycle violation implies global supercycle violation, and local strong connectedness violation implies global strong connectedness violation. The general global supercycle violation condition is the disjunction of global supercycle violation and global strong connectedness violation. Hence, we formulate the general local supercycle violation condition as the disjunction of local supercycle violation and local strong connectedness violation. It follows that the general local supercycle violation condition implies the general global supercycle violation condition.

Definition 7.3 (General local supercycle violation, genViolLoc ${ }_{\mathrm{D}}\left(v, s_{\mathrm{D}}\right)$ ). Let $v$ be an arbitrary

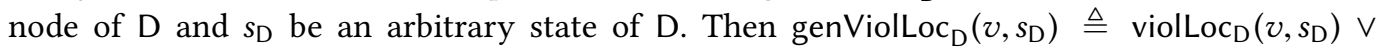
$\operatorname{sConnViolLoc}_{\mathrm{D}}\left(v, s_{\mathrm{D}}\right)$.

Proposition 7.4 (LocAl Violation IMPlies global violation). Let $s$ be an arbitrary state of BIP composite component $\mathrm{B}$. For all interactions $\mathrm{a} \in \gamma$, and $\ell \geq 1$, let $\mathrm{D}=\mathrm{D}_{\mathrm{a}}^{\ell}$ and $s_{\mathrm{D}}=s\lceil\mathrm{D}$. Also let $v$ be an arbitrary node of $\mathrm{D}$. Then $\operatorname{genViolLoc}_{\mathrm{D}}\left(v, s_{\mathrm{D}}\right)$ implies genViol $(v, s)$.

Proof. Assume that genViolLoc $\left(v, s_{\mathrm{D}}\right)$ holds. Then, by Definition 7.3, $\operatorname{viol}_{\mathrm{Loc}}\left(v, s_{\mathrm{D}}\right) \vee$ $\mathrm{sConnViolLoc}_{\mathrm{D}}\left(v, s_{\mathrm{D}}\right)$. We proceed by cases:

(1) $\operatorname{violLoc}_{\mathrm{D}}\left(v, s_{\mathrm{D}}\right)$ : hence $\operatorname{viol}_{\mathrm{B}}(v, s)$ by Proposition 6.23 .

(2) $\operatorname{sConnViolLoc}_{\mathrm{D}}\left(v, s_{\mathrm{D}}\right)$ : hence $\mathrm{sConnViol}_{\mathrm{B}}(v, s)$ by Proposition 7.2.

By Definition 5.3, genViol $(v, s) \triangleq \operatorname{viol}_{\mathrm{B}}(v, s) \vee \operatorname{sConnViol}_{\mathrm{B}}(v, s)$. Hence we conclude that genViol $_{\mathrm{B}}(v, s)$ holds.

7.1.3 Local AND-OR Condition. The actual local condition, $\mathcal{L} \mathcal{A} \mathcal{L} \mathcal{T}$, is given by applying the general local supercycle violation condition to every reachable transition of the subsystem $D$ being considered, and to every component $B_{i} \in$ components(a).

Definition $7.5\left(\mathcal{L} \mathcal{A} \mathcal{L} \mathcal{T}\left(\mathrm{B}, Q_{0}, \mathrm{a}, \ell\right)\right)$. Let $\ell \geq 1, \mathrm{D}=\mathrm{D}_{\mathrm{a}}^{\ell}, Q_{0}^{\mathrm{D}}=Q_{0}\left\lceil\mathrm{D}\right.$. Let $t_{\mathrm{D}} \stackrel{\mathrm{a}}{\rightarrow} s_{\mathrm{D}}$ be an arbitrary reachable transition of the subsystem $\left(\mathrm{D}, Q_{0}^{\mathrm{D}}\right)$. Then, in $s_{\mathrm{D}}$, the following holds. For every $\mathrm{B}_{i} \in$ components $(\mathrm{a}): \mathrm{B}_{i}$ has a general local supercycle violation that can be confirmed within $\mathrm{D}$. Formally,

$\forall \mathrm{B}_{i} \in$ components $(\mathrm{a}):$ genViolLoc $_{\mathrm{D}}\left(\mathrm{B}_{i}, s_{\mathrm{D}}\right)$.

We showed previously that $\mathcal{G} \mathcal{A} \mathcal{L} \mathcal{T}$ implies deadlock-freedom, and so it remains to establish that $\mathcal{L} \mathcal{A} \mathcal{L} \mathcal{T}$ implies $\mathcal{G} \mathcal{A} \mathcal{L} \mathcal{T}$.

Lemma 7.6. Let $\mathrm{a} \in \gamma$ be an interaction of BIP-system $\left(\mathrm{B}, Q_{0}\right)$. Then

$\left(\exists \ell \geq 1: \mathcal{L} \mathcal{A} \mathcal{L} \mathcal{T}\left(\mathrm{B}, Q_{0}, \mathrm{a}, \ell\right)\right)$ implies $\mathcal{G} \mathcal{A} \mathcal{L} \mathcal{T}\left(\mathrm{B}, Q_{0}, \mathrm{a}\right)$.

Proof. Assume $\mathcal{L} \mathcal{A} \mathcal{L} \mathcal{T}\left(\mathrm{B}, Q_{0}, \mathrm{a}, \ell\right)$ for some $\ell \geq 1$, and let $\mathrm{D}=\mathrm{D}_{\mathrm{a}}^{\ell}, Q_{0}^{\mathrm{D}}=Q_{0}\lceil\mathrm{D}$. Let $\stackrel{\mathrm{a}}{\rightarrow} s$ be an arbitrary reachable transition of BIP-system $\left(\mathrm{B}, Q_{0}\right)$, and let $t_{\mathrm{D}}=t\left|\mathrm{D}, s_{\mathrm{D}}=s\right| \mathrm{D}$, so that $t_{\mathrm{D}} \stackrel{\mathrm{a}}{\rightarrow} s_{\mathrm{D}}$ is the projection of $t \stackrel{\text { a }}{\rightarrow} s$ onto D. By Corollary $2.15, t_{\mathrm{D}} \stackrel{\text { a }}{\rightarrow} s_{\mathrm{D}}$ is a reachable transition of $\left(\mathrm{D}, Q_{0}^{\mathrm{D}}\right)$. By Definition 7.5,

for every reachable transition $t_{\mathrm{D}} \stackrel{\mathrm{a}}{\rightarrow} s_{\mathrm{D}}$ of $\left(\mathrm{D}, Q_{0}^{\mathrm{D}}\right)$ :

$\forall \mathrm{B}_{i} \in$ components $(\mathrm{a}):$ genViolLoc $_{\mathrm{D}}\left(\mathrm{B}_{i}, s_{\mathrm{D}}\right)$.

From this and Proposition 7.4,

for every reachable transition $t \stackrel{\mathrm{a}}{\rightarrow} s$ of $\left(\mathrm{B}, Q_{0}\right)$ :

$\forall \mathrm{B}_{i} \in \operatorname{components}(\mathrm{a}): \operatorname{genViol}_{\mathrm{B}}\left(\mathrm{B}_{i}, s\right)$

Hence, by Definition 5.4, $\mathcal{G} \mathcal{A} \mathcal{L} \mathcal{T}\left(\mathrm{B}, Q_{0}\right.$, a) holds. 
Table 1. Summary of predicates.

\begin{tabular}{|c|c|}
\hline $\operatorname{viol}_{\mathrm{B}}(v, s, d)$ & $v$ determined at depth $d$ to not be in supercycle \\
\hline $\operatorname{violLoc}_{\mathrm{D}}\left(v, s_{\mathrm{D}}, d\right)$ & $v$ locally determined at depth $d$ to not be in a supercycle \\
\hline $\operatorname{sConnViol}_{\mathrm{B}}(v, s)$ & $v$ not in a strongly connected supercycle \\
\hline $\operatorname{sConnViolLoc}_{\mathrm{D}}\left(v, s_{\mathrm{D}}\right)$ & $v$ locally determined to not be in a strongly connected supercycle \\
\hline $\operatorname{genViol}_{\mathrm{B}}(v, s)$ & $v$ does not contribute to a supercycle \\
\hline $\operatorname{genViolLoc}_{\mathrm{D}}\left(v, s_{\mathrm{D}}\right)$ & $v$ locally determined to not contribute to a supercycle \\
\hline
\end{tabular}

\section{Theorem 7.7. $\mathcal{L} \mathcal{A} \mathcal{L} \mathcal{T}$ is supercycle-freedom preserving.}

Proof. Follows immediately from Theorem 5.5 and Lemma 7.6.

Notice that Definition 7.5 calls genViolLoc $\left(v, s_{D}\right)$ on components, which by definition should be connected to at least one non-border interaction. As such, the trivial local supercycles, i.e., consisting only of border interactions, have no effect on supercycle formation.

\subsection{A local linear condition for deadlock-freedom}

We now formulate a local version of $\mathcal{G} \mathcal{L} \mathcal{I N}$. Observe that if in_depth $h_{\mathrm{B}}\left(\mathrm{B}_{i}, s\right)<\omega \vee$ out_depth $\mathrm{B}_{\mathrm{B}}\left(\mathrm{B}_{i}, s\right)<\omega$, then there is some finite $\ell$ such that in_depth $\left(\mathrm{B}_{i}, s\right)=\ell \mathrm{V}$ out_depth $\mathrm{B}_{\mathrm{B}}\left(\mathrm{B}_{i}, s\right)=\ell$.

Definition $7.8\left(\mathcal{L} \mathcal{L} \mathcal{I} \mathcal{N}\left(\mathrm{B}, Q_{0}, \mathrm{a}, \ell\right)\right)$. Let $\ell \geq 1, \mathrm{D}=\mathrm{D}_{\mathrm{a}}^{\ell}, Q_{0}^{\mathrm{D}}=Q_{0}\left\lceil\right.$ D. Let $t_{\mathrm{D}} \stackrel{\text { a }}{\rightarrow} s_{\mathrm{D}}$ be an arbitrary reachable transition of the subsystem $\left(\mathrm{D}, Q_{0}^{\mathrm{D}}\right)$. Then, in $s_{\mathrm{D}}$, the following holds. For every $\mathrm{B}_{i} \in$ components(a): either $\mathrm{B}_{i}$ has in-depth less than $2 \ell-1$, or out-depth less than $2 \ell-1$, in $W_{\mathrm{D}}\left(s_{\mathrm{D}}\right)$. Formally,

$$
\forall \mathrm{B}_{i} \in \text { components }(\mathrm{a}): \text { in_depth } \mathrm{D}_{\mathrm{D}}\left(\mathrm{B}_{i}, s_{\mathrm{D}}\right)<2 \ell-1 \vee \text { out_depth } \mathrm{D}_{\mathrm{D}}\left(\mathrm{B}_{i}, s_{\mathrm{D}}\right)<2 \ell-1 \text {. }
$$

To infer deadlock-freedom in (B, $\left.Q_{0}\right)$ by checking $\mathcal{L} \mathcal{L} \mathcal{I} N\left(\mathrm{~B}, Q_{0}, a, \ell\right)$, we use Proposition 6.2: since wait-for edges project up and down, it follows that wait-for paths project up and down, provided that the subsystem contains the entire wait-for path.

Proposition 7.9 (In-Projection, Out-Projection). Let $\ell \geq 1$, let $\mathrm{B}_{i}$ be an atomic component of $\mathrm{B}$, and let $\left(\mathrm{B}^{\prime}, Q_{0}^{\prime}\right)$ be a subsystem of $\left(\mathrm{B}, Q_{0}\right)$ which is based on a superset of $G_{\mathrm{a}}^{2 \ell}$. Let s be a state of $\left(B, Q_{0}\right)$, and $s^{\prime}=s\left\lceil B^{\prime}\right.$. Then (1) in_depth $\mathrm{B}_{\mathrm{B}}\left(\mathrm{B}_{i}, s\right)<2 \ell-1$ iff in_depth $\mathrm{B}^{\prime}\left(\mathrm{B}_{i}, s^{\prime}\right)<2 \ell-1$, and (2) out_depth $\mathrm{B}_{\mathrm{B}}\left(\mathrm{B}_{i}, s\right)<2 \ell-1$ iffout_depth $\mathrm{B}^{\prime}\left(\mathrm{B}_{i}, s^{\prime}\right)<2 \ell-1$.

Proof. We establish clause (1). The proof of clause (2) is analogous, except we replace paths ending in $\mathrm{B}_{i}$ by paths starting from $\mathrm{B}_{i}$. The proof of clause (1) is by double implication.

in_depth $\mathrm{B}_{\mathrm{B}}\left(\mathrm{B}_{i}, s\right)<2 \ell-1$ implies in_depth $\mathrm{B}^{\prime}\left(\mathrm{B}_{i}, s^{\prime}\right)<2 \ell-1:$ Assume that in_depth $\mathrm{B}_{\mathrm{B}}\left(\mathrm{B}_{i}, s\right)<$ $2 \ell-1$. Let $\pi$ be an arbitrary wait-for path in $W_{\mathrm{B}^{\prime}}\left(s^{\prime}\right)$ that ends in $\mathrm{B}_{i}$. Since $\left(\mathrm{B}^{\prime}, Q_{0}^{\prime}\right)$ is a subsystem of $\left(\mathrm{B}, Q_{0}\right)$, by Definition 3.3 and $s^{\prime}=s \backslash^{\prime}, W_{\mathrm{B}^{\prime}}\left(s^{\prime}\right)$ is a subgraph of $W_{\mathrm{B}}(s)$, i.e., $W_{\mathrm{B}^{\prime}}\left(s^{\prime}\right) \sqsubseteq W_{\mathrm{B}}(s)$. Hence $\pi$ is a wait-for path in $W_{\mathrm{B}}(s)$. By in_depth $h_{\mathrm{B}}\left(\mathrm{B}_{i}, s\right)<2 \ell-1$, we have $|\pi|<2 \ell-1$. Hence in_depth $h_{\mathrm{B}^{\prime}}\left(\mathrm{B}_{i}, s^{\prime}\right)<2 \ell-1$ since $\pi$ was arbitrarily chosen.

in_depth $\mathrm{B}^{\prime}\left(\mathrm{B}_{i}, s^{\prime}\right)<2 \ell-1$ implies in_depth $h_{\mathrm{B}}\left(\mathrm{B}_{i}, s\right)<2 \ell-1:$ Assume that in_depth $h_{\mathrm{B}}\left(\mathrm{B}_{i}, s\right) \geq$ $2 \ell-1$. Then there exists a wait-for path $\pi$ in $W_{\mathrm{B}}(s)$ such that $|\pi| \geq 2 \ell-1$ and $\pi$ ends in $\mathrm{B}_{i}$. Let $\rho$ be the suffix of $\pi$ with length $2 \ell-1$. Since $\left(\mathrm{B}^{\prime}, Q_{0}^{\prime}\right)$ is based on a superset of $G_{a}^{2 \ell}$, and since the distance from $\mathrm{B}_{i}$ to the border of $G_{\mathrm{a}}^{2 \ell}$ is $2 \ell-1$, we conclude that $\rho$ is a wait-for path that is wholly contained in $W_{\mathrm{B}^{\prime}}\left(s^{\prime}\right)$. Hence we have in_depth $h_{\mathrm{B}^{\prime}}\left(\mathrm{B}_{i}, s^{\prime}\right) \geq 2 \ell-1$. We have thus established 
in_depth $h_{\mathrm{B}}\left(\mathrm{B}_{i}, s\right) \geq 2 \ell-1$ implies in_depth $h_{\mathrm{B}^{\prime}}\left(\mathrm{B}_{i}, s^{\prime}\right) \geq 2 \ell-1$. The contrapositive is the desired result.

We now show that $\mathcal{L} \mathcal{L} \mathcal{I} \mathcal{N}\left(\mathrm{B}, Q_{0}, \mathrm{a}, \ell\right)$ implies $\mathcal{G} \mathcal{L} \mathcal{I} \mathcal{N}\left(\mathrm{B}, Q_{0}\right.$, a), which in turn implies deadlockfreedom.

Lemma 7.10. Let a be an interaction of BIP-system (B, $\left.Q_{0}\right)$, i.e., $\mathrm{B}=\gamma\left(\mathrm{B}_{1}, \ldots, \mathrm{B}_{n}\right)$ and $\mathrm{a} \in \gamma$. If $\mathcal{L} \mathcal{L} \mathcal{I}\left(\mathrm{B}, Q_{0}, \mathrm{a}, \ell\right)$ holds for some finite $\ell \geq 1$, then $\mathcal{G} \mathcal{L} \mathcal{I}\left(\mathrm{B}, Q_{0}\right.$, a) holds.

Proof. Let $t \stackrel{\mathrm{a}}{\rightarrow} s$ be a reachable transition of (B, $\left.Q_{0}\right)$ and let $\mathrm{B}_{i} \in \operatorname{components}(\mathrm{a})$. Let $\mathrm{D}=\mathrm{D}_{\mathrm{a}}^{\ell}$ and $Q_{0}^{\mathrm{D}}=Q_{0} \mid \mathrm{D}$. Let $s_{\mathrm{D}}=s \mid \mathrm{D}, t_{\mathrm{D}}=t\left\lceil\mathrm{D}\right.$. Then $t_{\mathrm{D}} \stackrel{\text { a }}{\rightarrow} s_{\mathrm{D}}$ is a reachable transition of $\left(\mathrm{D}, Q_{0}^{\mathrm{D}}\right)$ by Corollary 2.15. By $\mathcal{L} \mathcal{L} \mathcal{I N}\left(\mathrm{B}, Q_{0}, \mathrm{a}, \ell\right)$, in_depth $\mathrm{D}_{\mathrm{D}}\left(\mathrm{B}_{i}, s_{\mathrm{D}}\right)<2 \ell-1 \vee$ out_depth $_{\mathrm{D}}\left(\mathrm{B}_{i}, s_{\mathrm{D}}\right)<$ $2 \ell-1$. Hence by Proposition 7.9, in_depth in $\left._{i}, s\right)<2 \ell-1 \vee$ out_depth $\mathrm{B}_{\mathrm{B}}\left(\mathrm{B}_{i}, s\right)<2 \ell-1$. So in_depth $h_{\mathrm{B}}\left(\mathrm{B}_{i}, s\right)<\omega \vee$ out_depth $h_{\mathrm{B}}\left(\mathrm{B}_{i}, s\right)<\omega$. Hence $\mathcal{G} \mathcal{L} \mathcal{I N}\left(\mathrm{B}, Q_{0}, \mathrm{a}\right)$.

Proposition 7.11. Let $d<\ell$ and assume that node $v$ of $W_{\mathrm{D}}\left(s_{\mathrm{D}}\right)$ has finite out-depth of $d \geq 1$ in $W_{\mathrm{D}}\left(s_{\mathrm{D}}\right)$, i.e., out_depth $\left(v, s_{\mathrm{D}}\right)=d$. Then $\operatorname{violLoc}_{\mathrm{D}}\left(v, s_{\mathrm{D}}, d+1\right)$.

Proof. Proof is by induction on $d$.

Base case, $d=0$. Hence by out_depth $\left(v, s_{\mathrm{D}}\right)=0$, and Definitions 4.22 and 4.23 , $v$ has no outgoing wait-for edges in $W_{\mathrm{D}}\left(s_{\mathrm{D}}\right)$. By Definition 6.10, $\neg$ lblocks $s_{s_{\mathrm{D}}}\left(v, W_{\mathrm{D}}\left(s_{\mathrm{D}}\right)\right)$. By Definition 6.12, $v \in \mathcal{V} \mathcal{L}_{s_{\mathrm{D}}}(\emptyset)$. Hence violLoc $\mathrm{D}\left(v, s_{\mathrm{D}}, 1\right)$ by Definition 6.19 .

Induction step, $d>0$. Assume (out_depth $\left(v, s_{\mathrm{D}}\right)=d$ ). Let $w$ be an arbitrary successor of $v$, i.e., a node $w$ such that $v \rightarrow w \in W_{\mathrm{D}}\left(s_{\mathrm{D}}\right)$. By Definitions 4.22 and $4.23, w$ has an out-depth $d^{\prime}$ that is less than $d$.

By the induction hypothesis applied to $d^{\prime}$, we obtain violLoc $\operatorname{Li}_{\mathrm{D}}\left(w, s_{\mathrm{D}}, d^{\prime}+1\right)$, and so $w \in \mathcal{V} \mathcal{L}_{s_{\mathrm{D}}}^{d^{\prime}+1}(\emptyset)$ by Definition 6.19. Hence $w \in \mathcal{V} \mathcal{L}_{s_{\mathrm{D}}}^{d}(\emptyset)$, since, by monotonicity of $\mathcal{V} \mathcal{L}_{s_{\mathrm{D}}}$, we have $\mathcal{V} \mathcal{L}_{s_{\mathrm{D}}}^{n^{\prime}}(\emptyset) \sqsubseteq$ $\mathcal{V} \mathcal{L}_{s_{\mathrm{D}}}^{n}(\emptyset)$ when $n^{\prime} \leq n$. Since $w$ is an arbitrary successor of $v$, it follows that $v$ is only blocked by nodes in $\mathcal{V} \mathcal{L}_{s_{\mathrm{D}}}^{d}(\emptyset)$. Hence $\neg$ lblocks $s_{s_{\mathrm{D}}}\left(v, \widehat{\mathcal{V} \mathcal{L}_{s_{\mathrm{D}}}^{d}(\emptyset)}\right)$. By Definition 6.12, $v \in \mathcal{V} \mathcal{L}_{s_{\mathrm{D}}}\left(\mathcal{V} \mathcal{L}_{s_{\mathrm{D}}}^{d}(\emptyset)\right)$, i.e., $v \in \mathcal{V} \mathcal{L}_{s_{\mathrm{D}}}^{d+1}(\emptyset)$. By Definition 6.19, $\operatorname{viol}_{\operatorname{Loc}}\left(v, s_{\mathrm{D}}, d+1\right)$.

Lemma 7.12. For all interactions a of $\mathrm{B}$, i.e., $\mathrm{a} \in \gamma$, $\mathcal{L} \mathcal{L} \mathcal{I} \mathcal{N}\left(\mathrm{B}, Q_{0}, \mathrm{a}, \ell\right)$ implies $\mathcal{L} \mathcal{A} \mathcal{L} \mathcal{T}\left(\mathrm{B}, Q_{0}, \mathrm{a}, \ell\right)$.

Proof. Assume $\mathcal{L} \mathcal{L} \mathcal{I} \mathcal{N}\left(\mathrm{B}, Q_{0}, \mathrm{a}, \ell\right)$. Let $t_{\mathrm{D}} \stackrel{\mathrm{a}}{\rightarrow} s_{\mathrm{D}}$ be an arbitrary reachable transition of $\mathrm{D}$, and let $\mathrm{B}_{i}$ be an arbitrary component of components(a). Then, from Definition 7.8, we have:

$$
\text { in_depth } h_{\mathrm{D}}\left(\mathrm{B}_{i}, s_{\mathrm{D}}\right)<2 \ell-1 \vee \text { out_depth }\left(\mathrm{B}_{i}, s_{\mathrm{D}}\right)<2 \ell-1 \text {. }
$$

The proof proceeds by two cases.

in_depth ${ }_{\mathrm{D}}\left(\mathrm{B}_{i}, s_{\mathrm{D}}\right)<2 \ell-1$ : Hence $\mathrm{B}_{i}$ cannot be in a strongly connected supercycle, because $\mathrm{B}_{i}$ would then lie on at least one wait-for cycle, and so would have infinite in-depth. Hence sConnViolLoc $_{\mathrm{D}}\left(\mathrm{B}_{i}, s_{\mathrm{D}}\right)$ by Definition 7.1, Clause 1. Hence by Definition 7.3, genViolLoc ${ }_{\mathrm{D}}\left(\mathrm{B}_{i}, s_{\mathrm{D}}\right)$.

out_depth $_{\mathrm{D}}\left(\mathrm{B}_{i}, s_{\mathrm{D}}\right)<2 \ell-1$ : Hence out_depth $\left(\mathrm{B}_{i}, s_{\mathrm{D}}\right)=d$ for some $d<2 \ell-1$. By Proposition 7.11, violLoc ${ }_{\mathrm{D}}\left(\mathrm{B}_{i}, s_{\mathrm{D}}, d+1\right)$. Hence by Definition 7.3, genViolLoc ${ }_{\mathrm{D}}\left(\mathrm{B}_{i}, s_{\mathrm{D}}\right)$.

In both cases, we have genViolLoc ${ }_{\mathrm{D}}\left(\mathrm{B}_{i}, s_{\mathrm{D}}\right)$. Since $\mathrm{B}_{i}$ is an arbitrarily chosen component of components(a), we have $\forall \mathrm{B}_{i} \in$ components $(\mathrm{a}): \operatorname{genViolLoc}_{\mathrm{D}}\left(\mathrm{B}_{i}, s_{\mathrm{D}}\right)$. Hence, by Definition 7.5, we conclude $\mathcal{L} \mathcal{A} \mathcal{L} \mathcal{T}\left(\mathrm{B}, Q_{0}, \mathrm{a}, \ell\right)$. 
TheOREM 7.13. $\mathcal{L} \mathcal{L} \mathcal{I} \mathcal{N}$ is supercycle-freedom preserving

Proof. Follows immediately from Theorem 5.9 and Lemma 7.10. Also follows immediately from Theorem 5.5 and Lemma 7.12.

\section{OVERALL SOUNDNESS, COMPLETENESS, AND IMPLICATION RESULTS}

Figure 9 gives the implication relations between our four deadlock-freedom conditions. Each implication arrow is labeled by the Lemma that provides the corresponding result.

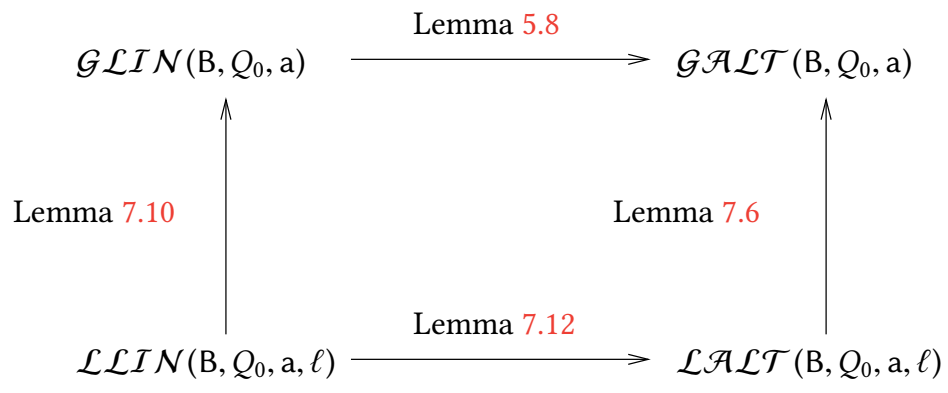

Fig. 9. Implication relations between deadlock-freedom conditions.

We can use the four conditions together: if, for each interaction, we verify one of the conditions, then we can infer deadlock-freedom, i.e., combining the conditions in this manner is still sound w.r.t. deadlock-freedom.

Theorem 8.1 (Deadlock-freedom via $\mathcal{G} \mathcal{A} \mathcal{L} \mathcal{T}, \mathcal{G} \mathcal{L} \mathcal{I}, \mathcal{L} \mathcal{A} \mathcal{L} \mathcal{T}, \mathcal{L} \mathcal{L} \mathcal{I}$ ). Assume that

(1) for all $s_{0} \in Q_{0}, W_{\mathrm{B}}\left(s_{0}\right)$ is supercycle-free, and

(2) for all interactions a of $\mathrm{B}$ (i.e., a $\in \gamma$ ), one of the following holds:
(a) $\mathcal{G} \mathcal{A} \mathcal{L} \mathcal{T}\left(\mathrm{B}, Q_{0}\right.$, a)
(b) $\mathcal{G} \mathcal{L} \mathcal{I} \mathcal{N}\left(\mathrm{B}, Q_{0}, \mathrm{a}\right)$
(c) $\exists \ell \geq 1: \mathcal{L} \mathcal{A} \mathcal{L} \mathcal{T}\left(\mathrm{B}, Q_{0}, \mathrm{a}, \ell\right)$
(d) $\exists \ell \geq 1: \mathcal{L} \mathcal{L} \mathcal{I} \mathcal{N}\left(\mathrm{B}, Q_{0}, \mathrm{a}, \ell\right)$

Then for every reachable state $u$ of $\left(\mathrm{B}, Q_{0}\right): W_{\mathrm{B}}(u)$ is supercycle-free, and so $\left(\mathrm{B}, Q_{0}\right)$ is free of local and global deadlock.

Proof. Immediate from Theorems 5.5, 5.9, 7.7, 7.13 and Corollary 3.14.

Finally, we establish that $\mathcal{G} \mathcal{A} \mathcal{L} \mathcal{T}$ is complete w.r.t. deadlock-freedom: any system that is free of local and global deadlock will satisfy $\mathcal{G} \mathcal{A} \mathcal{L} \mathcal{T}$.

Theorem 8.2 (COMPLeteness of $\mathcal{G} \mathcal{A} \mathcal{L} \mathcal{T}$ w.r.T. DEAdlock-Freedom). Assume that (B, $\left.Q_{0}\right)$ is free from local and global deadlock. Then, for all interactions a of $\mathrm{B}$ (i.e., a $\in \gamma), \mathcal{G} \mathcal{A} \mathcal{L} \mathcal{T}\left(\mathrm{B}, Q_{0}, \mathrm{a}\right)$ holds.

Proof. Let a be an arbitrary interaction in $\gamma$, and let $t \stackrel{\text { a }}{\rightarrow} s$ be a reachable transition of (B, $\left.Q_{0}\right)$. Hence $s$ is a reachable state of $\left(\mathrm{B}, Q_{0}\right)$. Suppose that $W_{\mathrm{B}}(s)$ contains a supercycle $S C$. Then, by Proposition 3.8, the subcomponent $\mathrm{B}^{\prime}$ consisting of all the atomic components $\mathrm{B}_{i} \in S C$ cannot execute a transition from any state reachable from $s$, and so is deadlocked. Hence $\left(\mathrm{B}, Q_{0}\right)$ has a local deadlock in reachable state $s$, contrary to assumption. Hence $W_{\mathrm{B}}(s)$ is supercycle-free. 
Let $v$ be an arbitrary node in $W_{\mathrm{B}}(s)$. By Definition $4.17, \neg s c y c_{\mathrm{B}}(v, s)$ holds. Hence by Proposition 4.18, $\operatorname{viol}_{\mathrm{B}}(v, s)$ holds. By Definition 5.3, genViol $(v, s)$ holds. Since $v$ is an arbitrary node in $W_{\mathrm{B}}(s)$, and all $\mathrm{B}_{i} \in$ components $(\mathrm{a})$ are nodes in $W_{\mathrm{B}}(s)$, we have $\left(\forall \mathrm{B}_{i} \in\right.$ components(a), genViol $\left(\mathrm{B}_{i}, s\right)$ ). By Definition $5.4, \mathcal{G} \mathcal{A} \mathcal{L} \mathcal{T}\left(\mathrm{B}, Q_{0}, \mathrm{a}\right)$ holds. Since a is an arbitrary interaction in $\gamma$, we have $\left(\forall \mathrm{a} \in \gamma: \mathcal{G} \mathcal{A} \mathcal{L} \mathcal{T}\left(\mathrm{B}, Q_{0}, \mathrm{a}\right)\right)$, and the theorem is established.

\section{IMPLEMENTATION AND EXPERIMENTS}

To implement our deadlock-freedom conditions, we must:

(1) Check that all initial states are supercycle-free

(2) Evaluate $\mathcal{L} \mathcal{A} \mathcal{L} \mathcal{T}$

(3) Evaluate $\mathcal{L} \mathcal{L} \mathcal{I} \mathcal{N}$

Tasks 1 and 2 require the computation of $l f p\left(\mathcal{V} \mathcal{L}_{s_{\mathrm{D}}}\right)$. Figure 10 presents an algorithm that does this. Its correctness follows immediately from Definitions 6.10 and 6.12 .

\section{Compute-LFP(D, $\left.s_{\mathrm{D}}\right)$}

$\triangleright$ Precondition: $s_{\mathrm{D}}$ is a state of $\mathrm{D}$

$\triangleright$ Postcondition: returns least fixpoint of $\mathcal{V} \mathcal{L}_{s_{\mathrm{D}}}$ i.e., $\operatorname{lfp}\left(\mathcal{V} \mathcal{L}_{s_{\mathrm{D}}}\right)=\bigsqcup_{d \geq 1} \mathcal{V} \mathcal{L}_{s_{\mathrm{D}}}^{d}(\emptyset)$

1. $X \leftarrow \emptyset$;

2. while (true)

3. $\triangleright$ loop invariant: at end of $i$ 'th iteration, $X=\mathcal{V} \mathcal{L}_{s_{\mathrm{D}}}^{i}(\emptyset)=\bigsqcup_{1 \leq d \leq i} \mathcal{V} \mathcal{L}_{s_{\mathrm{D}}}^{d}(\emptyset)$

4. $\quad Y \leftarrow$ Compute-VL $\left(\mathrm{D}, s_{\mathrm{D}}, X\right)$;

5. if $(X=Y) \operatorname{return}(X)$;

6. $X \leftarrow Y$

7. endwhile

Fig. 10. Procedure to compute $l f p\left(\mathcal{V} \mathcal{L}_{S_{\mathrm{D}}}\right)$.

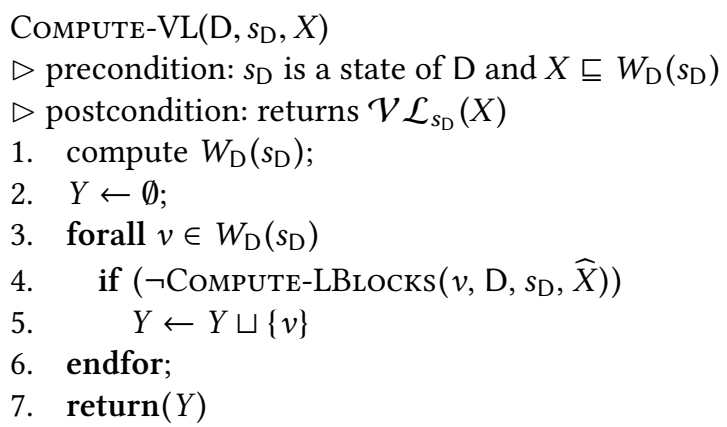

Fig. 11. Procedure to compute $\mathcal{V} \mathcal{L}_{S_{\mathrm{D}}}(X)$. 


\section{Compute-LBlocks $\left(v, \mathrm{D}, s_{\mathrm{D}}, X\right)$}

$\triangleright$ precondition: $s_{\mathrm{D}}$ is a state of $\mathrm{D}, X \sqsubseteq W_{\mathrm{D}}\left(s_{\mathrm{D}}\right)$, and $v \in W_{\mathrm{D}}\left(s_{\mathrm{D}}\right)$

$\triangleright$ postcondition: returns lblocks $_{s_{\mathrm{D}}}(v, X)$

1. if $(v$ is a border interaction of $\mathrm{D})$

2. $\quad$ return $($ true $) \quad \triangleright$ border interaction pessimistically assumed to be blocked

3. else if $\left(v\right.$ is an interior interaction a and $\left.\left(\exists \mathrm{B}_{i} \in X: \mathrm{a} \rightarrow \mathrm{B}_{i} \in W_{\mathrm{D}}\left(s_{\mathrm{D}}\right)\right)\right)$

$\triangleright$ some outgoing wait-for edge goes to a component in $X$

5. $\quad$ else if $\left(v\right.$ is a component $\mathrm{B}_{i}$ and $\left.\left(\forall \mathrm{a}: \mathrm{B}_{i} \rightarrow \mathrm{a} \in W_{\mathrm{D}}\left(s_{\mathrm{D}}\right) \Rightarrow \mathrm{a} \in X\right)\right)$

6. return $($ true $) \quad$ bevery outgoing wait-for edge goes to some interaction in $X$

7. else

8. $\quad$ return $($ false $)$

9. fi

$\triangleright$ all cases for blocking do not hold, cf. Definition 6.10

Fig. 12. Procedure to compute lblocks $_{s_{\mathrm{D}}}(v, X)$.

Note that $Y \sqcup\{v\}$ is the join of the wait-for-subgraph $Y$ with the wait-for-subgraph $v$ consisting of the single node $v$. Recall that the edges of $Y \sqcup\{v\}$ are induced from $W_{\mathrm{D}}\left(s_{\mathrm{D}}\right)$.

Let $\operatorname{Deg}_{v}$ be the outdegree of $v$ in $W_{\mathrm{D}}\left(t_{\mathrm{D}}\right)$, $\mid$ nodes $(\mathrm{D}) \mid$ be the number of components and interactions in $\mathrm{D}$, i.e., the number of nodes in $W_{\mathrm{D}}\left(s_{\mathrm{D}}\right)$ and $|\mathrm{D}|$ be the size of the syntactic description of D. We assume that membership in $X$ and $Y$ can be determined in constant time, e.g., by using Boolean arrays, and that evaluation of transition guards in components takes time proportional to the length of the guards. Then, the time complexity of Compute-LBlocks $\left(v, \mathrm{D}, s_{\mathrm{D}}, X\right)$ is $O\left(\operatorname{Deg}_{v}\right)$. Hence the time complexity of Compute-VL $\left(\mathrm{D}, s_{\mathrm{D}}, X\right)$ is $O\left(|\mathrm{D}|+\left|W_{\mathrm{D}}\left(t_{\mathrm{D}}\right)\right|\right)$, since each edge in $W_{\mathrm{D}}\left(t_{\mathrm{D}}\right)$ is examined at most once, over all calls of Compute-LBLOcKs $\left(v, \mathrm{D}, s_{\mathrm{D}}, X\right)$, and computing $W_{\mathrm{D}}\left(t_{\mathrm{D}}\right)$ can be done in time $O(|\mathrm{D}|)$. Since $\left|W_{\mathrm{D}}\left(t_{\mathrm{D}}\right)\right|$ is $O(|\mathrm{D}|)$, this is just $O(|\mathrm{D}|)$. The time complexity of COMPUTE-LFP $\left(\mathrm{D}, s_{\mathrm{D}}\right)$ is then $O(F i x \cdot|\mathrm{D}|)$ where Fix is the number of iterations that Compute-LFP $\left(\mathrm{D}, s_{\mathrm{D}}\right)$ takes to reach a fixpoint. Fix is $O(\mid$ nodes $(\mathrm{D}) \mid)$, since each iteration either adds a node or reaches the least fixpoint. Thus the time complexity of Compute-LFP(D, $\left.s_{\mathrm{D}}\right)$ is $O(|\operatorname{nodes}(\mathrm{D})| \cdot|\mathrm{D}|)$.

Theorem 8.1 requires that all initial states be supercycle-free. We assume that the number of initial states is small, so that we can check each explicitly. Figure 13 presents an algorithm which checks that all initial states are supercycle-free.

\section{CHECKINITSUPERCYCLEFrEe $\left(Q_{0}\right)$}

$\triangleright$ returns true iff all initial states are supercycle-free

1. forall $s_{0} \in Q_{0}$

2. compute $W_{\mathrm{B}}\left(s_{0}\right)$

3. $U \leftarrow$ COMPUTE-LFP $\left(\mathrm{B}, s_{0}\right)$

4. if $\left(U \neq W_{\mathrm{B}}\left(s_{0}\right)\right)$ then return(false)

5. endfor;

6. return(true)

$\triangleright s_{0}$ not supercycle-free, so return false

$\triangleright$ all initial states are supercycle-free

Fig. 13. Procedure to check that all initial states are supercycle-free.

Proposition 9.1. CHECKINITSUPERCyCLEFREe $\left(Q_{0}\right)$ returns true iff all initial states are supercyclefree. 
Proof. Consider the execution of checkInitSupercycleFree $\left(Q_{0}\right)$ for an arbitrary $s_{0} \in Q_{0}$.

By its construction, $U \sqsubseteq W_{\mathrm{B}}\left(s_{0}\right)$. Suppose that $U \neq W_{\mathrm{B}}\left(s_{0}\right)$. By Propositions 4.18 and 4.9 , the nodes of $W_{\mathrm{B}}\left(s_{0}\right)$ that are not in $U$ constitute a supercycle. Hence $s_{0}$ not supercycle-free, and so false is the correct result in this case.

Now suppose that $U=W_{\mathrm{B}}\left(s_{0}\right)$. Hence every node in $W_{\mathrm{B}}\left(s_{0}\right)$ has a supercycle violation, and so by Proposition 4.18, no node of $W_{\mathrm{B}}\left(s_{0}\right)$ is in a supercycle. Hence $W_{\mathrm{B}}\left(s_{0}\right)$ does not contain a supercycle, and so $s_{0}$ is supercycle-free. Hence the for loop continues on to the next initial state. If all initial states are supercycle-free, the for loop terminates, and cHEcKInITSUPERCyCLEFreE $\left(Q_{0}\right)$ returns true, as required.

Let $|\mathrm{B}|$ be the size of the syntactic description of $\mathrm{B}, \mid$ nodes $(\mathrm{B}) \mid$ be the number of components and interactions in $\mathrm{B}$, which is also the number of nodes in $W_{\mathrm{B}}\left(s_{0}\right)$, and $\left|Q_{0}\right|$ be the number of states in $Q_{0}$, i.e., the number of initial states, Then time complexity of CHeckINITSUPERCyCLeFreE $\left(Q_{0}\right)$ is $O\left(\left|Q_{0}\right| \cdot|\operatorname{nodes}(\mathrm{B})| \cdot|\mathrm{B}|\right)$.

\subsection{Implementation of the linear condition $\mathcal{L} \mathcal{L} \mathcal{N}$}

Figure 14 presents the pseudocode for our algorithm $\operatorname{LLin}\left(\mathrm{B}, Q_{0}\right)$ to evaluate $\mathcal{L} \mathcal{L} \mathcal{I}$. LLin $\left(\mathrm{B}, Q_{0}\right)$ iterates over each interaction a of $\left(\mathrm{B}, Q_{0}\right)$, and invokes $\operatorname{LLinINT}\left(\mathrm{B}, Q_{0}, \mathrm{a}\right)$ to evaluate $(\exists \ell \geq$ $\left.1: \mathcal{L} \mathcal{L} \mathcal{I} N\left(\mathrm{~B}, Q_{0}, \mathrm{a}, \ell\right)\right)$. LLinInt(B, $\left.Q_{0}, \mathrm{a}\right)$ starts with $\ell=1$ and increments $\ell$ until either $\mathcal{L} \mathcal{L} \mathcal{I}\left(\mathrm{B}, Q_{0}, \mathrm{a}, \ell\right)$ is found to hold, or $\mathrm{D}$ has become the entire system and $\mathcal{L} \mathcal{L} \mathcal{I} \mathcal{N}\left(\mathrm{B}, Q_{0}, \mathrm{a}, \ell\right)$ does not hold. In the latter case, $\mathcal{L} \mathcal{L} \mathcal{I} N\left(\mathrm{~B}, Q_{0}, \mathrm{a}, \ell\right)$ does not hold for any finite $\ell$, and, in practice, computation would halt before $\mathrm{D}$ had become the entire system, due to exhaustion of resources. Evaluation of $\mathcal{L} \mathcal{L} \mathcal{I} \mathcal{N}\left(\mathrm{B}, Q_{0}, \mathrm{a}, \ell\right)$ is done by $\operatorname{LLinInTDist}\left(\mathrm{B}, Q_{0}, \mathrm{a}, \ell\right)$, which examines every reachable transition that executes a, and checks that the final state satisfies Definition 7.8. 
$\operatorname{LLin}\left(\mathrm{B}, Q_{0}\right)$, where $\mathrm{B} \triangleq \gamma\left(\mathrm{B}_{1}, \ldots, \mathrm{B}_{n}\right)$

$\triangleright$ returns true iff $\left(\forall \mathrm{a} \in \gamma, \exists \ell \geq 1: \mathcal{L} \mathcal{L} \mathcal{I} \mathcal{N}\left(\mathrm{B}, Q_{0}, \mathrm{a}, \ell\right)\right)$

1. forall interactions $a \in \gamma$

2. if $\left(\operatorname{LLinInt}\left(\mathrm{B}, Q_{0}, \mathrm{a}\right)=\right.$ false $) \operatorname{return}($ false $) \mathrm{fi}$

3. endfor;

4. return(true)

$\triangleright$ return true if check succeeds for all $\mathrm{a} \in \gamma$

$\operatorname{LLinINT}\left(\mathrm{B}, Q_{0}, \mathrm{a}\right)$, where $\mathrm{B} \triangleq \gamma\left(\mathrm{B}_{1}, \ldots, \mathrm{B}_{n}\right), \mathrm{a} \in \gamma$

$\triangleright$ returns true iff $\left(\exists \ell \geq 1: \mathcal{L} \mathcal{L} \mathcal{I} \mathcal{N}\left(\mathrm{B}, Q_{0}, \mathrm{a}, \ell\right)\right)$

1. $\ell \leftarrow 1$;

$\triangleright$ start with $\ell=1$

2. while (true)

3. if (LLinIntDist $\left(\mathrm{B}, Q_{0}, \mathrm{a}, \ell\right)=$ true) return(true) $\mathrm{fi}$;

$\triangleright$ success, so return true

4. if $\left(\mathrm{D}_{\mathrm{a}}^{\ell}=\gamma\left(\mathrm{B}_{1}, \ldots, \mathrm{B}_{n}\right)\right)$ return(false $) \mathrm{fi}$; $\quad \triangleright$ exhausted all subsystems, return false

5. $\ell \leftarrow \ell+1 \quad \triangleright$ increment $\ell$ until success (true) or intractable or failure (false)

6. endwhile

$\operatorname{LLinIntDist}\left(\mathrm{B}, Q_{0}, \mathrm{a}, \ell\right)$

$\triangleright$ returns true iff $\left.\mathcal{L} \mathcal{L} \mathcal{I} \mathcal{N}\left(\mathrm{B}, Q_{0}, \mathrm{a}, \ell\right)\right)$

1. let $\mathrm{D}=\mathrm{D}_{\mathrm{a}}^{\ell}$

2. forall reachable transitions $t_{\mathrm{D}} \stackrel{\mathrm{a}}{\rightarrow} s_{\mathrm{D}}$ of $\mathrm{D}$

3. compute $W_{\mathrm{D}}\left(s_{\mathrm{D}}\right)$;

4. if $\left(\neg\left(\forall \mathrm{B}_{i} \in\right.\right.$ components $(\mathrm{a}):$ in_depth $_{\mathrm{D}}\left(\mathrm{B}_{i}, s_{\mathrm{D}}\right)<2 \ell-1 \vee$ out_depth $\left.\left.\mathrm{D}_{\mathrm{D}}\left(\mathrm{B}_{i}, s_{\mathrm{D}}\right)<2 \ell-1\right)\right)$

5. $\quad \operatorname{return}($ false $)$

$\triangleright$ check Definition 7.8

6. $\mathrm{fi}$

7. endfor;

8. return(true)

$\triangleright$ return true if check succeeds for all transitions

Fig. 14. Pseudocode for the implementation of $\mathcal{L} \mathcal{L} \mathcal{I}$.

Time complexity. Let $\ell_{\mathrm{a}}$ be the smallest value of $\ell$ for which $\mathcal{L} \mathcal{L} \mathcal{I} \mathcal{N}\left(\mathrm{B}, Q_{0}, \mathrm{a}, \ell\right)$ holds, $M_{\mathrm{a}}^{\ell}$ be the transition system of $\mathrm{D}_{\mathrm{a}}^{\ell},\left|M_{\mathrm{a}}^{\ell}\right|$ be the size (number of nodes plus number of edges) of $M_{\mathrm{a}}^{\ell}$, and $\left|\mathrm{D}_{\mathrm{a}}^{\ell}\right|$ be the size of the syntactic description of $\mathrm{D}_{\mathrm{a}}^{\ell}$. Then the running time of $\operatorname{LLinInTDisT}\left(\mathrm{B}, Q_{0}, \mathrm{a}, \ell\right)$ is $O\left(\left|M_{\mathrm{a}}^{\ell}\right| \cdot\left|\mathrm{D}_{\mathrm{a}}^{\ell}\right|\right)$, since computing $W_{\mathrm{D}_{\mathrm{a}}^{\ell}}\left(s_{\mathrm{D}}\right)$ can be done in time $O\left(\left|\mathrm{D}_{\mathrm{a}}^{\ell}\right|\right)$, and $W_{\mathrm{D}_{\mathrm{a}}^{\ell}}\left(s_{\mathrm{D}}\right)$ has size in $O\left(\left|\mathrm{D}_{\mathrm{a}}^{\ell}\right|\right)$, and computing in-depth and out-depth in $W_{\mathrm{D}_{\mathrm{a}}^{\ell}}\left(s_{\mathrm{D}}\right)$ can be done in linear time using depth-first graph search. The running time of $\operatorname{LLinINT}\left(\mathrm{B}, Q_{0}, \mathrm{a}\right)$, is $O\left(\Sigma_{1 \leq \ell \leq \ell_{\mathrm{a}}}\left|M_{\mathrm{a}}^{\ell}\right| \cdot\left|\mathrm{D}_{\mathrm{a}}^{\ell}\right|\right)$. The running time of $\operatorname{LLin}\left(\mathrm{B}, Q_{0}\right)$ is $O\left(\sum_{\mathrm{a} \in \gamma} \sum_{1 \leq \ell \leq \ell_{\mathrm{a}}}\left|M_{\mathrm{a}}^{\ell}\right| \cdot\left|\mathrm{D}_{\mathrm{a}}^{\ell}\right|\right)$.

\subsection{Implementation of the AND-OR condition $\mathcal{L} \mathcal{A} \mathcal{L} \mathcal{T}$}

Figure 15 presents the pseudocode for our algorithm $\operatorname{LALT}\left(\mathrm{B}, Q_{0}\right)$ to evaluate $\mathcal{L} \mathcal{A} \mathcal{L} \mathcal{T}$. This uses the Compute-LFP $\left(D, s_{\mathrm{D}}\right)$ algorithm for computing local supercycle violations in $\mathrm{D}$, given in Figure 10 .

$\operatorname{LALT}\left(\mathrm{B}, Q_{0}\right)$ iterates over each interaction a of (B, $\left.Q_{0}\right)$, and invokes $\operatorname{LatTINT}\left(\mathrm{B}, Q_{0}\right.$, a) to evaluate $\left(\exists \ell \geq 1: \mathcal{L} \mathcal{A} \mathcal{L} \mathcal{T}\left(\mathrm{B}, Q_{0}\right.\right.$, a,$\left.\left.\ell\right)\right)$. LALtint $\left(\mathrm{B}, Q_{0}\right.$, a) starts with $\ell=1$ and increments $\ell$ until either $\mathcal{L} \mathcal{A} \mathcal{L} \mathcal{T}\left(\mathrm{B}, Q_{0}, \mathrm{a}, \ell\right)$ is found to hold, or $\mathrm{D}$ has become the entire system and $\mathcal{L} \mathcal{A} \mathcal{L} \mathcal{T}\left(\mathrm{B}, Q_{0}, \mathrm{a}, \ell\right)$ does not hold. In the latter case, $\mathcal{L} \mathcal{A} \mathcal{L} \mathcal{T}\left(\mathrm{B}, Q_{0}, \mathrm{a}, \ell\right)$ does not hold for any finite $\ell$, and, in practice, computation would halt before $\mathrm{D}$ had become the entire system, due to exhaustion of resources. Note that $D$ is the smallest system in which a supercycle-violation can be confirmed. 
Table 2. Summary of procedures.

\begin{tabular}{|c|c|}
\hline $\operatorname{LALT}\left(\mathrm{B}, Q_{0}\right)$ & true iff $\left(\forall \mathrm{a} \in \gamma, \exists \ell \geq 1: \mathcal{L} \mathcal{A} \mathcal{L} \mathcal{T}\left(B, Q_{0}, \mathrm{a}, \ell\right)\right)$ \\
\hline $\operatorname{LALTINT}\left(\mathrm{B}, Q_{0}, \mathrm{a}\right)$ & true iff $\left(\exists \ell \geq 1: \mathcal{L} \mathcal{A} \mathcal{L} \mathcal{T}\left(B, Q_{0}, a, \ell\right)\right)$ \\
\hline $\operatorname{LALTINTDist}\left(\mathrm{B}, Q_{0}, \mathrm{a}, \ell\right)$ & true iff $\mathcal{L} \mathcal{A} \mathcal{L} \mathcal{T}\left(\mathrm{B}, Q_{0}, \mathrm{a}, \ell\right)$ \\
\hline GenLocViol $\left(\mathrm{B}_{i}, V, \mathrm{D}, s_{\mathrm{D}}\right)$ & $\begin{array}{l}\text { true iff } B_{i} \text { has local sc-formation violation } \\
\text { in state } s_{\mathrm{D}} \text { of } \mathrm{D} \text {, i.e., genViolLoc } \mathrm{D}\left(B_{i}, s_{\mathrm{D}}\right) \text { holds }\end{array}$ \\
\hline $\operatorname{LOCScONNScVIOL}\left(\mathrm{B}_{i}, V, \mathrm{D}, s_{\mathrm{D}}\right)$ & $\begin{array}{l}\text { true iff } B_{i} \text { has local strong connectedness violation } \\
\text { in } t_{\mathrm{a}} \text {, i.e., } \mathrm{s} \text { ConnViolLoc } \mathrm{D}\left(B_{i}, s_{\mathrm{D}}\right) \text { holds }\end{array}$ \\
\hline COMPUTE-LFP(D, $\left.s_{\mathrm{D}}\right)$ & $\begin{array}{l}\text { compute local supercycle violations } \\
\text { in state } s_{\mathrm{D}} \text { of } \mathrm{D} \text {, i.e., violLoc } \mathrm{D}_{\mathrm{D}}\left(v, s_{\mathrm{D}}, d\right) \text { for all } v, d\end{array}$ \\
\hline
\end{tabular}

Evaluation of $\mathcal{L} \mathcal{A} \mathcal{L} \mathcal{T}\left(\mathrm{B}, Q_{0}, \mathrm{a}, \ell\right)$ is done by $\operatorname{LaltintDist}\left(\mathrm{B}, Q_{0}, \mathrm{a}, \ell\right)$, which invokes Compute-LFP $\left(\mathrm{D}, t_{\mathrm{D}}\right)$ to compute the local supercycle violations and $\operatorname{GenLocVioL}\left(\mathrm{B}_{i}, V, \mathrm{D}, s_{\mathrm{D}}\right)$ to compute the general local supercycle violations. GenLocViol $\left(\mathrm{B}_{i}, V, \mathrm{D}, s_{\mathrm{D}}\right)$ invokes LOCSCONNScVIOL $\left(\mathrm{B}_{i}, V, \mathrm{D}, s_{\mathrm{D}}\right)$ to compute the local strong connectedness violation. The pseudocode is a straightforward translation of Definitions 7.1 and 7.3. Table 2 shows a summary of the procedures.

Time complexity. Let $\ell_{\mathrm{a}}$ be the smallest value of $\ell$ for which $\mathcal{L} \mathcal{A} \mathcal{L} \mathcal{T}\left(\mathrm{B}, Q_{0}\right.$, a,$\left.\ell\right)$ holds, $M_{\mathrm{a}}^{\ell}$ be the transition system of $\mathrm{D}_{\mathrm{a}}^{\ell},\left|M_{\mathrm{a}}^{\ell}\right|$ be the size (number of nodes plus number of edges) of $M_{\mathrm{a}}^{\ell},\left|\mathrm{D}_{\mathrm{a}}^{\ell}\right|$ be the size of the syntactic description of $\mathrm{D}_{\mathrm{a}}^{\ell}$, and $\left|\operatorname{nodes}\left(\mathrm{D}_{\mathrm{a}}^{\ell}\right)\right|$ be the number of components and interactions in $\mathrm{D}_{\mathrm{a}}^{\ell}$. The time complexity of LocSconnScVioL $\left(\mathrm{B}_{i}, V, \mathrm{D}_{\mathrm{a}}^{\ell}, s_{\mathrm{D}}\right)$ is $O\left(\left|\operatorname{nodes}\left(\mathrm{D}_{\mathrm{a}}^{\ell}\right)\right| \cdot\left|\mathrm{D}_{\mathrm{a}}^{\ell}\right|\right)$, since maximal strongly connected components are computable in linear time using Tarjan's algorithm [30], and the existence of paths in $W L$ from $B_{i}$ to the border of $D_{a}^{\ell}$ can be checked in linear time by using depth-first graph search. Also, checking for supercycles (via least-fixpoint computation) within the strongly connected component $C$ can be done in time $O\left(\left|\operatorname{nodes}\left(\mathrm{D}_{\mathrm{a}}^{\ell}\right)\right| \cdot\left|\mathrm{D}_{\mathrm{a}}^{\ell}\right|\right)$, amortized over all such $C$. The time complexity of $\operatorname{GenLocVioL}\left(\mathrm{B}_{i}, V, \mathrm{D}_{\mathrm{a}}^{\ell}, s_{\mathrm{D}}\right)$ is also $O\left(\left|\operatorname{nodes}\left(\mathrm{D}_{\mathrm{a}}^{\ell}\right)\right| \cdot\left|\mathrm{D}_{\mathrm{a}}^{\ell}\right|\right)$. The running time of $\operatorname{LaLt} \operatorname{IntDist}\left(\mathrm{B}, Q_{0}, \mathrm{a}, \ell\right)$ is $O\left(\left|M_{\mathrm{a}}^{\ell}\right| \cdot\left|\operatorname{nodes}\left(\mathrm{D}_{\mathrm{a}}^{\ell}\right)\right| \cdot\left|\mathrm{D}_{\mathrm{a}}^{\ell}\right|\right)$, since Compute-LFP $\left(\mathrm{D}_{\mathrm{a}}^{\ell}, s_{\mathrm{D}}\right)$ has time complexity in $O\left(\mid\right.$ nodes $\left.\left(\mathrm{D}_{\mathrm{a}}^{\ell}\right)|\cdot| \mathrm{D}_{\mathrm{a}}^{\ell} \mid\right)$, and computing $W_{\mathrm{D}_{\mathrm{a}}^{\ell}}\left(s_{\mathrm{D}}\right)$ can be done in time $O\left(\left|\mathrm{D}_{\mathrm{a}}^{\ell}\right|\right)$. The running time of $\operatorname{LaLt} \operatorname{Int}\left(\mathrm{B}, Q_{0}, \mathrm{a}\right)$ is $O\left(\Sigma_{1 \leq \ell \leq \ell_{\mathrm{a}}}\left|M_{\mathrm{a}}^{\ell}\right| \cdot\left|\operatorname{nodes}\left(\mathrm{D}_{\mathrm{a}}^{\ell}\right)\right| \cdot\left|\mathrm{D}_{\mathrm{a}}^{\ell}\right|\right)$ since $\operatorname{LattInt}\left(\mathrm{B}, Q_{0}\right.$, a) iterates $\operatorname{LattintDist}\left(\mathrm{B}, Q_{0}, \mathrm{a}, \ell\right)$ until $\ell=\ell_{\mathrm{a}}$. The running time of $\operatorname{Latt}\left(\mathrm{B}, Q_{0}\right)$ is $O\left(\sum_{\mathrm{a} \in \gamma} \sum_{1 \leq \ell \leq \ell_{\mathrm{a}}}\left|M_{\mathrm{a}}^{\ell}\right| \cdot\left|\operatorname{nodes}\left(\mathrm{D}_{\mathrm{a}}^{\ell}\right)\right| \cdot\left|\mathrm{D}_{\mathrm{a}}^{\ell}\right|\right)$ since $\operatorname{LaLt}\left(\mathrm{B}, Q_{0}\right)$ calls $\operatorname{Lattint}\left(\mathrm{B}, Q_{0}\right.$, a) for every a $\in \gamma$.

\subsection{Tool-set}

We provide LALT-BIP, a suite of supporting tools that implement our method. LALT-BIP consists of about 2500 Java lines of code LALT-BIP is equipped with a command line interface (see Figure 16) that accepts a set of configuration options. It takes the name of the input BIP file and other optional flags.

\subsection{Experimentation}

We evaluated LALT-BIP using several case studies including the dining philosopher example and multiple instances of a configurable generalized Resource Allocation System that comprises a configurable multi token-based scheduler. The different configurations of our resource allocation system subsume problems like the Milner's scheduler, data arbiters and the dining philosopher with a butler problem. We benchmarked the performance of LALT-BIP against DFinder [13] on two benchmarks: Dining Philosopher with an increasing number of philosophers and a deadlock free resource allocation system with an increasing number of clients and resources. 
$\operatorname{LALT}\left(\mathrm{B}, Q_{0}\right)$, where $\mathrm{B} \triangleq \gamma\left(\mathrm{B}_{1}, \ldots, \mathrm{B}_{n}\right)$

$\triangleright$ returns true iff $\left(\forall \mathrm{a} \in \gamma, \exists \ell \geq 1: \mathcal{L} \mathcal{A} \mathcal{L} \mathcal{T}\left(\mathrm{B}, Q_{0}, \mathrm{a}, \ell\right)\right)$

1. forall interactions $a \in \gamma$

2. if $\left(\operatorname{Lattint}\left(\mathrm{B}, Q_{0}, \mathrm{a}\right)=\right.$ false $) \operatorname{return}($ false $) \mathrm{fi}$

3. endfor;

4. $\operatorname{return}($ true $)$

$\triangleright$ return true if check succeeds for all $a \in \gamma$

$\operatorname{LALTINT}\left(\mathrm{B}, Q_{0}, \mathrm{a}\right)$, where $\mathrm{B} \triangleq \gamma\left(\mathrm{B}_{1}, \ldots, \mathrm{B}_{n}\right), \mathrm{a} \in \gamma$

$\triangleright$ returns true iff $\left(\exists \ell \geq 1: \mathcal{L} \mathcal{A} \mathcal{L} \mathcal{T}\left(\mathrm{B}, Q_{0}, \mathrm{a}, \ell\right)\right)$

1. $\ell \leftarrow 1$;

$\triangleright$ start with $\ell=1$

2. while (true)

3. if $\left(\operatorname{LaLtINTDist}\left(\mathrm{B}, Q_{0}, \mathrm{a}, \ell\right)=\right.$ true $)$ return(true) $\mathbf{f}$;

$\triangleright$ success, so return true

4. if $\left(\mathrm{D}_{\mathrm{a}}^{\ell}=\gamma\left(\mathrm{B}_{1}, \ldots, \mathrm{B}_{n}\right)\right)$ return(false) $\mathrm{f}$;

ll subsystems, return false

5. $\ell \leftarrow \ell+1 \quad \triangleright$ increment $\ell$ until success or intractable or failure

6. endwhile

LALtintDist(B, $\left.Q_{0}, \mathrm{a}, \ell\right)$

$\triangleright$ returns true iff $\mathcal{L} \mathcal{A} \mathcal{L} \mathcal{T}\left(\mathrm{B}, Q_{0}, \mathrm{a}, \ell\right)$

1. let $\mathrm{D}=\mathrm{D}_{\mathrm{a}}^{\ell}$

2. forall reachable transitions $t_{\mathrm{D}} \stackrel{\mathrm{a}}{\rightarrow} s_{\mathrm{D}}$ of $\mathrm{D}$

3. compute $W_{\mathrm{D}}\left(s_{\mathrm{D}}\right)$;

4. $\quad V \leftarrow$ COMPUTE-LFP $\left(\mathrm{D}, s_{\mathrm{D}}\right)$;

$\triangleright$ see Figure 10

5. forall $\mathrm{B}_{i} \in$ components $(\mathrm{a})$

6. if $\left(\neg \operatorname{GenLocVIOL}\left(\mathrm{B}_{i}, V, \mathrm{D}, s_{\mathrm{D}}\right)\right)$ return $($ false $) \mathrm{fi} \quad \triangleright$ no violation for $\mathrm{B}_{i}$

7. endfor

8. endfor;

9. return(true)

$\triangleright$ all $\mathrm{B}_{i} \in$ components(a) have a general local supercycle violation

GenLocViol $\left(\mathrm{B}_{i}, V, \mathrm{D}, s_{\mathrm{D}}\right)$

$\triangleright$ returns true iff genViolLoc ${ }_{\mathrm{D}}\left(\mathrm{B}_{i}, s_{\mathrm{D}}\right)$ holds (Definition 7.3)

$\triangleright$ i.e., $\mathrm{B}_{i}$ has a general local supercycle violation in state $s_{\mathrm{D}}$ of subsystem $\mathrm{D}$

1. $\operatorname{return}\left(\mathrm{B}_{i} \in V \vee \operatorname{LOCSCONNScVIOL}\left(\mathrm{B}_{i}, V, \mathrm{D}, s_{\mathrm{D}}\right)\right)$

LOCSCONNScVIOL $\left(\mathrm{B}_{i}, V, \mathrm{D}, s_{\mathrm{D}}\right)$

$\triangleright$ returns true iff $\mathrm{s}$ ConnViolLoc ${ }_{\mathrm{D}}\left(\mathrm{B}_{i}, s_{\mathrm{D}}\right)$ holds (Definition 7.1)

$\triangleright$ i.e., $\mathrm{B}_{i}$ has a local strong connectedness violation in state $s_{\mathrm{D}}$ of subsystem $\mathrm{D}$

1. $W L \leftarrow W_{\mathrm{D}}\left(s_{\mathrm{D}}\right)-V$, i.e., remove from $W_{\mathrm{D}}\left(s_{\mathrm{D}}\right)$ all nodes with a local supercycle violation;

2. compute maximal strongly connected components of $W L$;

3. forall maximal strongly connected components $C$ of $W L$

4. if ( $C$ contains a non-trivial strongly connected supercycle which contains $\mathrm{B}_{i}$ as a node)

5. $\quad \operatorname{return}($ false) $\mathrm{fi}$; $\quad \triangleright$ Definition 7.1, Clause 1 violated

6. if (there is no path in $W L$ from $B_{i}$ to a border node of $\mathrm{D}$ )

7. return(true) fi;

8. if (there is no path in $W L$ from some border node of $D$ to $B_{i}$ )

9. $\quad$ return(true) fi;

10. $\operatorname{return}($ false $)$

$\triangleright$ Definition 7.1, Clause 2a holds

$\triangleright$ Definition 7.1, Clause 2b holds $\triangleright$ Definition 7.1, Clauses 1 and 2 both violated

Fig. 15. Pseudocode for the implementation of $\mathcal{L} \mathcal{A} \mathcal{L} \mathcal{T}$. 


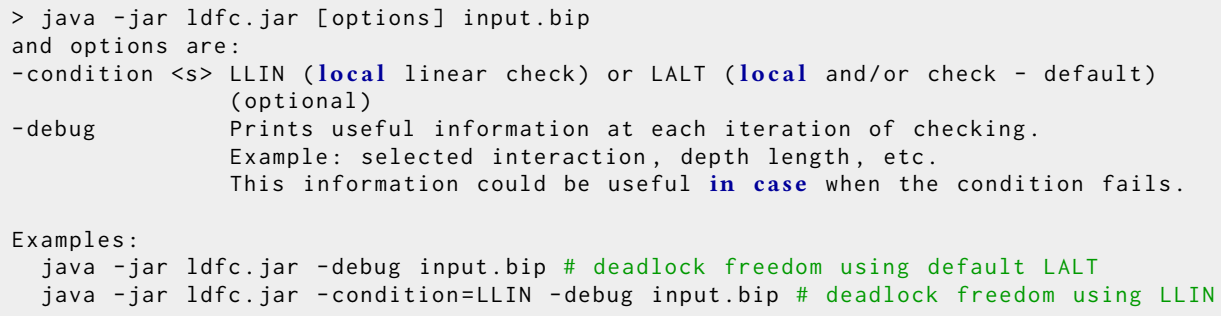

Fig. 16. LALT-BIP Command Line Interface.

All experiments were conducted on a machine with Intel (R) 8-Cores (TM) i7-6700, CPU @ 3.40GHZ, 32GB RAM, running a CentOS Linux distribution.

9.4.1 Dining philosophers case study. We consider the traditional dining philosopher problem as depicted in Figure 1, which shows 4 philosophers and 4 forks modeled in BIP.

Each philosopher component has 2 states, and each fork component has 3 states. Thus, the total number of states is $2^{n} \times 3^{n}$. We evaluated LALT-BIP by increasing $n$ and applying both $\mathcal{L} \mathcal{A} \mathcal{L}$ and $\mathcal{L} \mathcal{L} \mathcal{N}$ methods and compared against the best configuration we could compute with DFinder2. DFinder2 allows for several techniques to be applied. The most efficient one is the Incremental Positive Mapping (IPM) technique [13]. IPM requires a manual partitioning of the system to exploit its efficiency. We applied IPM on all structural partitions and we report on the best result which is consistent with the results reported in [13].

Table 3 shows the results. Both $\mathcal{L} \mathcal{A} \mathcal{L} \mathcal{T}$ and $\mathcal{L} \mathcal{L} \mathcal{I}$ outperform the best performance of DFinder2 by several orders of magnitude for $n \leq 3,000$. Both $\mathcal{L} \mathcal{A} \mathcal{L} \mathcal{T}$ and $\mathcal{L} \mathcal{L} \mathcal{I}$ successfully completed the deadlock freedom check for $3,000 \leq n \leq 10,000$ in less than one minute, where DFinder2 timed out (1 Hour). The sole exception being that $\mathcal{L} \mathcal{L} \mathcal{I} N$ required 62 seconds for $n=10,000$.

Even though $\mathcal{L} \mathcal{L} \mathcal{I} N$ is asymptotically more efficient than $\mathcal{L} \mathcal{A} \mathcal{L} \mathcal{T}, \mathcal{L} \mathcal{A} \mathcal{L} \mathcal{T}$ outperforms $\mathcal{L} \mathcal{L} \mathcal{N}$ in all cases. This due to the following.

- The largest subsystem that $\mathcal{L} \mathcal{A} \mathcal{L} \mathcal{T}$ had to consider was with depth $\ell=1$. This corresponds to $18=2^{1} \times 3^{2}$ states regardless of $n$, the number of philosophers.

- The largest subsystem that $\mathcal{L} \mathcal{L} \mathcal{I} N$ had to consider was with depth $\ell=2$. This corresponds to $648=2^{3} \times 3^{4}$ states regardless of $n$.

- For a given depth $\ell, \mathcal{L} \mathcal{L} \mathcal{I} N$ is more efficient to compute than $\mathcal{L} \mathcal{A} \mathcal{L} \mathcal{T}$. Since $\mathcal{L} \mathcal{A} \mathcal{L} \mathcal{T}$ performs a stronger check, it often terminates for smaller depths, which makes it more efficient than $\mathcal{L} \mathcal{L} \mathcal{I} N$ in many cases.

9.4.2 Resource allocation system case studies. We evaluated LALT-BIP with a multi token-based resource allocation system. The system consists of $n$ clients, $m$ resources, $k$ tokens. The number of tokens specifies the maximum number of resources that can be in use at a given time. The system allows to specify conflicting resources. Only one resource out of a set of conflicting resources can be in use at a given time. For each set of conflicting resources, we create a resource manager. Resource managers are connected in a ring where they pass tokens to neighboring resource managers or to resources.

Given a configuration specifying $n, m, k$, a map of requests between clients and resources, and a set of sets of conflicting resources, we automatically generate a corresponding BIP model.

Figures 17, 18, and 19 show BIP atomic components for client, resource and manager components. 
Table 3. Benchmarks: Dining Philosopher (seconds or minutes : seconds).

\begin{tabular}{|llll|}
\hline Size & $\mathcal{L} \mathcal{A} \mathcal{L} \mathcal{T}$ & $\mathcal{L} \mathcal{L} \mathcal{N}$ & D-Finder \\
\hline 1,000 & 0.46 & 0.7 & 15 \\
2,000 & 1.4 & 1.9 & 60 \\
3,000 & 2.9 & 4 & $2: 41$ \\
4,000 & 4.8 & 7 & $5: 37$ \\
5,000 & 8.3 & 12 & $12: 38$ \\
6,000 & 13.0 & 17 & $17: 48$ \\
7,000 & 17.2 & 25 & $30: 18$ \\
8,000 & 25.6 & 34 & - \\
9,000 & 34.1 & 55 & - \\
10,000 & 47 & 62 & - \\
\hline
\end{tabular}

The client in Figure 17 requests resources $R_{0}$ and $R_{2}$ in sequence. It has 5 ports. Ports $S R_{0}$ and $S R_{2}$ send requests for resources $R_{0}$ and $R_{2}$, respectively. Ports $R G_{0}$ and $R G_{2}$ receive grants for resources $R_{0}$ and $R_{2}$, respectively. Port rel releases all resources. The behavior of the client depends on its request sequence.

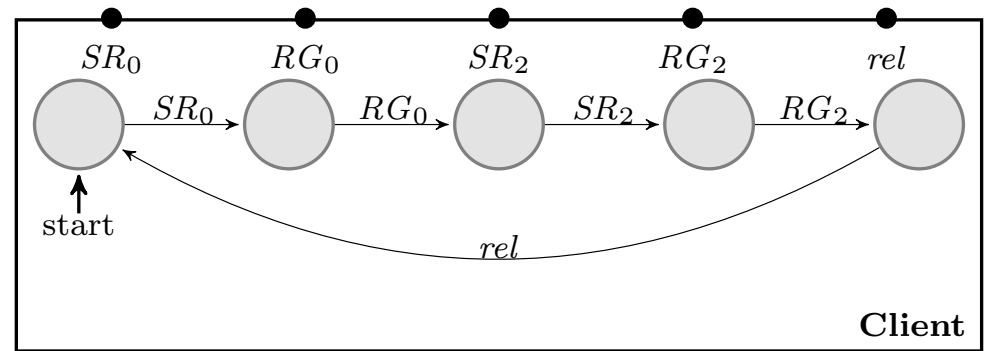

Fig. 17. Client.

Figure 18 shows a resource component. A resource component waits for a request from a connected client on port $R R$. Once a request is received, the resource component transitions to a state where it is ready to receive a token from the corresponding resource manager using port $R T T$. The resource transitions to a state where it grants the client request using port STC and waits until it is released on port done. There, it returns the token back to the resource manager and transitions to the start state. 


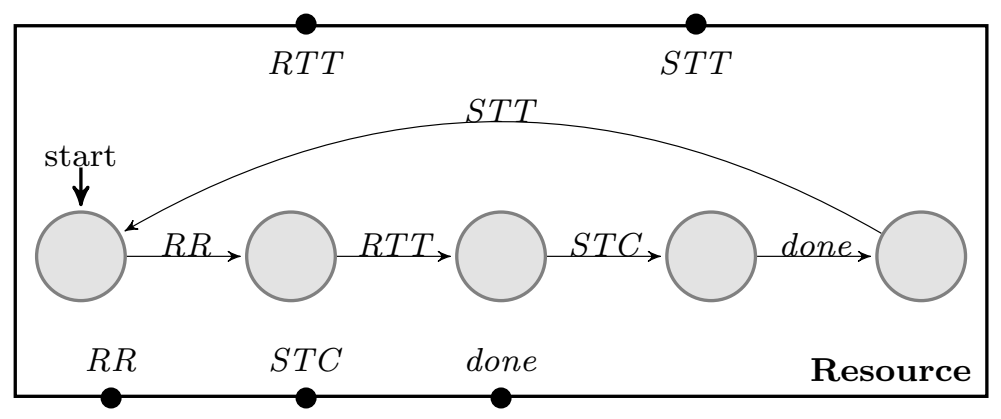

Fig. 18. Resource.

Figure 19 shows a resource manager. A resource manager $M$ has four states.

- State $T$ denotes that $M$ has a token. $M$ may send the token to either (1) a resource on port $S T R$ and transition to state $T w R$ (token with resource), or (2) the next resource manager on port STT and transition to state $N$ (no token).

- State $N$ denotes that $N$ has no token. It may receive a token from a neighboring resource manager in the ring on port $R T T$ and transition to state $T$.

- State $T w R$ denotes that $M$ has already passed a token to one of its resources. $M$ may either receive (1) the assigned token back from the resource using port $R T R$ and transition to state $T$, or (2) another token from a neighboring manager using port $R T T$ and transition to state $T T w R$ (token and token with resource).

- State $T T w R$ denotes that $M$ has a token and has already passed a token to one of its resources. In this state $M$ cannot send the token it has to a resource it manages to respect the conflict constraint. $M$ may send the token to the next manager on port STT and transition back to state $T w R$.

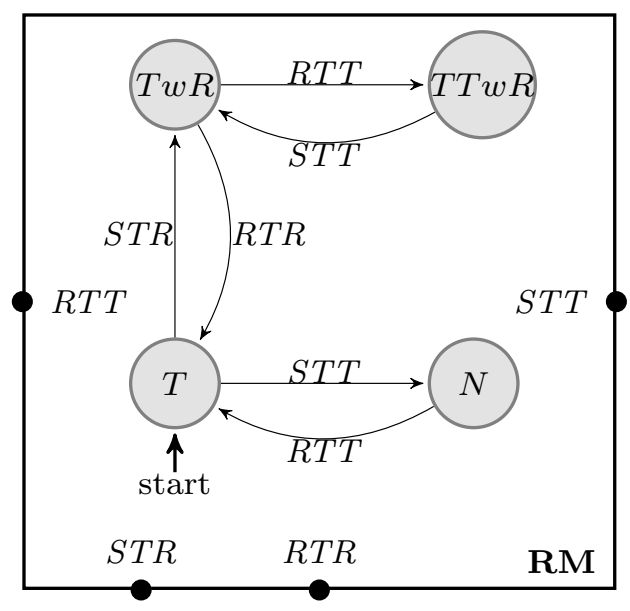

Fig. 19. Token Resource Manager.

The connections between a resource manager $M$ and its resources on ports STR and RTR specify that the resources are conflicting. A system should have at least $x$ resource managers where $x$ is 
the maximum between the number of sets of conflicting resources and $k$. Note that $k$ resource managers start at state $T$ to denote the $k$ tokens; the rest start at state $N$.

Figure 20 shows a configuration system with 5 clients and 5 resources where:

- Client $C_{0}$ requires resource $R_{0}$ then $R_{2}$,

- Client $C_{1}$ requires resource $R_{2}$ then $R_{0}$,

- Client $C_{2}$ requires resource $R_{1}$,

- Client $C_{3}$ requires resource $R_{3}$, and

- Client $C_{4}$ requires resource $R_{4}$.

The system has three resource managers to specify the conflicting resources. $R M_{01}$ manages conflicting resources $\left\{R_{0}, R_{1}\right\} . R M_{23}$ managers conflicting resources $\left\{R_{2}, R_{3}\right\} . R M_{4}$ manages resource $R_{4}$.

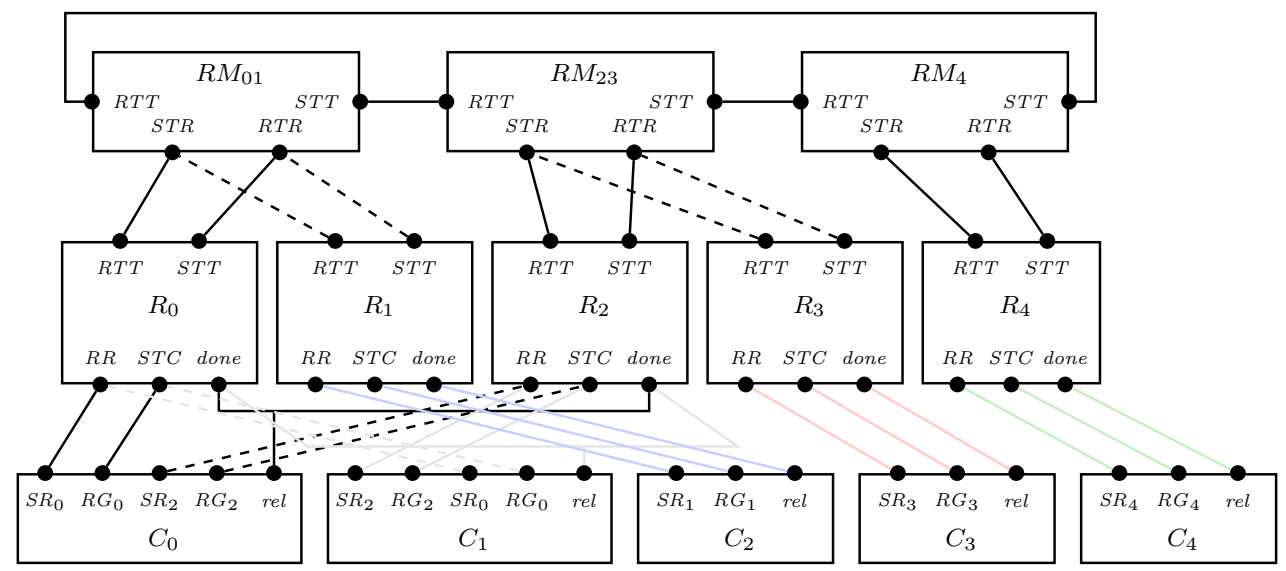

Fig. 20. Conflict-Resource Allocation System.

We evaluated LALT-BIP with various configurations. We highlight several lessons learned for specific systems as follows.

Lesson 1. $\mathcal{L} \mathcal{A} \mathcal{L} \mathcal{T}$ verifies freedom from global and local deadlock where DFinder2 can only verify freedom from global deadlock. Consider a system with 5 clients, 3 tokens, and 5 resources. Clients request resources $\langle 0,2\rangle,\langle 2,0\rangle,\langle 1\rangle,\langle 3\rangle$, and $\langle 4\rangle$, respectively. Resource sets $\{0,1\},\{2,3\}$ are conflicting. This system is clearly global deadlock free. It has a local deadlock where client $C_{0}$ has resource 0 and client $C_{1}$ has resource 2 . DFinder qualitatively can not detect such a local deadlock while $\mathcal{L} \mathcal{A} \mathcal{L} \mathcal{T}$ successfully does.

Lesson 2. $\mathcal{L} \mathcal{A} \mathcal{L} \mathcal{T}$ is more complete than both $\mathcal{L} \mathcal{L} \mathcal{I}$ and DFinder2. For example, it can verify global and local deadlock freedom in cases where $\mathcal{L} \mathcal{L} \mathcal{I} \mathcal{N}$ fails. Consider a system with 5 clients, 2 tokens, and 5 resources. Clients request resources $\langle 0,2\rangle,\langle 0,2\rangle,\langle 1\rangle,\langle 3\rangle$, and $\langle 4\rangle$, respectively. Resource sets $\{0,1\},\{2,3,4\}$ are conflicting. This system is global and local deadlock free. Both DFinder2 and $\mathcal{L} \mathcal{L} \mathcal{I}$ report that the system might contain a deadlock. $\mathcal{L} \mathcal{A} \mathcal{L} \mathcal{T}$ successfully reports that the system is both global and local deadlock free.

Benchmarking: We evaluated the performance of $\mathcal{L} \mathcal{A} \mathcal{L} \mathcal{T}$ on a deadlock free system with the following configuration.

- $n$ clients each with 3 states, $n$ resources each with 5 states, and $n$ tokens, 
Table 4. Benchmarks: Time required for $\mathcal{L} \mathcal{A} \mathcal{L} \mathcal{T}$ on the resource allocation system

\begin{tabular}{|lccccccccccc|}
\hline Size & 10 & 12 & 14 & 16 & 18 & 20 & 22 & 24 & 26 & 28 & 30 \\
Time (sec) & 148 & 169 & 189 & 230 & 254 & 277 & 298 & 318 & 351 & 374 & 430 \\
\hline
\end{tabular}

- Client $C_{i}, 0 \leq i<n$ requests resource $i$, and

- No resources are in conflict, hence we have $n$ resource managers each with 4 states.

The system has a total of $4^{n} \times 3^{n} \times 5^{n}$ states. DFinder2 timed out within seven hours for $n=10$. $\mathcal{L} \mathcal{L} \mathcal{N}$ had to increase the subsystem up to the whole system and also timed out within seven hours for $n=10$. $\mathcal{L} \mathcal{A} \mathcal{L} \mathcal{T}$ was able to verify deadlock freedom. It has to check subsystems with 12 components out of $3 \times n$ components regardless of $n$. This resulted from inspecting subsystems corresponding to a depth $\ell=2$ with $\leq 23,040,000=4^{6} \times 3^{2} \times 5^{4}$ states regardless of $n$. The numbers in Table 4 show a linear increase in time required to check deadlock freedom using $\mathcal{L} \mathcal{A} \mathcal{L} \mathcal{T}$ with respect to $n$. This indicates that the number of subsystems to check is proportional to $n$.

Our resource allocation system subsumes the token based Milner scheduler [25] which is essentially a token ring with precisely one token present [3].

\section{RELATED WORK, DISCUSSION, AND FURTHER WORK}

The notions of wait-for-graph and supercycle [7, 8] were initially defined for a shared memory program $P=P_{1}\|\cdots\| P_{K}$ in pairwise normal form [4, 5]: a binary symmetric relation $I$ specifies the directly interacting pairs ("neighbors") $\left\{P_{i}, P_{j}\right\}$ If $P_{i}$ has neighbors $P_{j}$ and $P_{k}$, then the code in $P_{i}$ that interacts with $P_{j}$ is expressed separately from the code in $P_{i}$ that interacts with $P_{k}$. These synchronization codes are executed synchronously and atomically, so the grain of atomicity is proportional to the degree of $I$. [7] give two polynomial time methods for (local and global) deadlock freedom. The first checks subsystems consisting of three processes. The second computes the waitfor-graphs of all pair subsystems $P_{i} \| P_{j}$, and takes their union, for all pairs and all reachable states of each pair. The first method considers only wait-for-paths of length $\leq 2$. The second method is prone to false negatives, because wait-for edges generated by different states are all merged together, which can result in spurious supercycles.

[21] use a BIP-like formalism, Interaction Models. They present a criterion for global deadlock freedom, based on an and-or graph with components and constraints as the two sets of nodes. A constraint gives the condition under which a component is blocked. Edges are labeled with conjuncts of the constraints. Deadlock freedom is checked by traversing every cycle, taking the conjunction of all the conditions labeling its edges, and verifying that this conjunction is always false, i.e., verifying the absence of cyclical blocking. No complexity bounds are given. [23] present a polynomial time checkable deadlock freedom condition based on structural restrictions: "the communication structure between the components is given by a tree." This restriction allows them to analyze only pair systems. [2] use a formalism based on process algebra. They check deadlock by analyzing cycles in the connections between software components, and claim scalability, but no complexity bounds are given.

[29] present several rules for freedom of global deadlock of "triple disjoint" (no action involves $>2$ processes) CSP concurrent programs. The basis for these rules is to first check that each individual process is deadlock free (i.e., the network is "busy"), and then to define a "variant function" that maps the state of each process to a partially ordered set. The first rule requires to establish that, if $P_{i}$ waits for $P_{j}$, then the value of $P_{i}$ 's state is greater than the value of $P_{j}$ 's state. Since every process is blocked in a global deadlock, one can then construct an infinite sequence of processes with strictly decreasing values, which are therefore all distinct. This cannot happen in a finite 
network, and hence some process is not blocked. They treat several examples, including a self-timed systolic array (in 2 and 3 dimensions), dining philosophers, and a message switching network. They generalize the first rule to exploit "disconnecting edges" (whose removal partitions the network into disconnected components) to decompose the proof of deadlock freedom into showing that each disconnected component is deadlock-free, and also to weaken the restriction on the variant function so that it only has to decrease for at least one edge on each wait-for cycle. [17] also provide criteria for deadlock freedom of triple-disjoint CSP programs, and use the same technical framework as [29]. However, they do not use variant functions, but show that, in a busy network, a deadlock implies the existence of a wait-for cycle. They give many examples, and demonstrate the absence of wait-for cycles in each example, by ad-hoc reasoning. Finally, they give a deadlock freedom rule that exploits disconnecting edges, similar to that of [29]. In both of these papers, the wait-for relations are defined by examining a pair of processes at a time: $P_{i}$ waits for $P_{j}$ iff $P_{i}$ offers an action to $P_{j}$ which $P_{j}$ is not willing to participate in.

[24] applies the results of [29] and [17] to formulate deadlock-freedom design rules for several classes of CSP concurrent programs: cyclic processes, client-server protocols, and resource allocation protocols. He also introduces the notion of "state dependence digraph" (SDD), whose nodes are local states of individual processes, and whose edges are wait-for relations between processes in particular local states. An acyclic SDD implies deadlock-freedom. A cyclic SDD does not imply deadlock, however, since the cycle may be "spurious": the local states along the cycle may not be reachable at the same time, and so the cycle cannot give rise to an actual deadlock during execution. Hence the SDD approach cannot deal with "non-hereditary" deadlock freedom, i.e., a deadlock free system that contains a deadlock prone subsystem. Consider, e.g.,, the dining philosophers with a butler solution; removing the butler leaves a deadlock prone subsystem. [3] takes the SDD approach and improves its accuracy by checking for mutual reachability of pairs of local states, and also eliminating local states and pairs of local states, where action enablement can be verified locally These checks are formulated as a Boolean formula which is then sent to a SAT solver. Their method is able to verify deadlock freedom of dining philosophers with a butler, whereas our method timed out, since the subsystems on which $\mathcal{L} \mathcal{A} \mathcal{L} \mathcal{T}\left(\mathrm{B}, Q_{0}, \mathrm{a}, \ell\right)$ is evaluated becomes the entire system On the other hand, our approach succeeded in quickly verifying deadlock-freedom of the resource allocation example, whereas the method of [3] failed for Milner's token based scheduler, which is a special case of our resource allocation example. An intriguing topic for future work is to attempt to combine the two methods, to obtain the advantages of both.

We compared our implementation LALT-BIP to D-Finder 2 [13]. D-Finder 2 computes a finitestate abstraction for each component, which it uses to compute a global invariant $I$. It then checks if I implies deadlock freedom. Unlike LALT-BIP, D-Finder 2 handles infinite state systems. However, LALT-BIP had superior running time for dining philosophers and resource controller (both finitestate).

All the above methods (except [7]) verify global (and not local) deadlock-freedom. Our method verifies local deadlock-freedom, which subsumes global deadlock-freedom as a special case. Also, our approach makes no structural restriction at all on the system being checked for deadlock. Our method checks for the absence of supercycles, which are a sound and complete characterization of deadlock. Moreover, the $\mathcal{L} \mathcal{A} \mathcal{L} \mathcal{T}$ condition is complete w.r.t. the occurrence of a supercycle wholly within the subsystem being checked, and the $\mathcal{G} \mathcal{A} \mathcal{L} \mathcal{T}$ condition is complete w.r.t. freedom from local and global deadlock, as given by Theorem 8.2. None of the above papers give a completeness result similar to Theorem 8.2. Hence, the only source of incompleteness in our method is that of computational limitation: if the subsystem being checked becomes too large before the $\mathcal{L} \mathcal{A} \mathcal{L} \mathcal{T}$ condition is verified. If computational resources are not exhausted, then our method can keep checking until the subsystem being checked is the entire system, at which point $\mathcal{L} \mathcal{A} \mathcal{L} \mathcal{T}$ coincides 
with $\mathcal{G} \mathcal{A} \mathcal{L} \mathcal{T}$, which is sound and complete for local deadlock (Proposition 4.18, Definition 5.3, and Definition 5.4).

Related to methods that specifically check for deadlock-freedom are methods that check for general safety properties, e.g., using abstraction and compositional reasoning. The work of [31] extends CTL* with constructs to support expressing and distinguishing between deadlock, livelock, and successful termination. This is key since the standard semantics of CTL* requires that Kripke structures be total, i.e., every state has at least one outgoing transition, and so deadlock cannot be modeled. They provide a semantics for $\mathrm{CTL}^{*}$ in which deadlock, livelock, and successful termination can all be distinguished.

Abstraction methods related to compositional reasoning [1, 19, 28] that target safety properties can be used to prove global deadlock freedom. The aforementioned papers target parameterized systems composed of $N$ communicating processes $P_{i}, 1 \leq i \leq N$. They over-approximate the reachable state space of a system with $N$ processes, using symbolic states from a system of size $K<N$. If the property holds for the system of size $K$, then it holds for any arbitrary $N$. Crucial to [1] is a bound on the number of processes involved at each state, such that the post image (typically infinite) can be computed using successor operations of size $K+\ell$ where $\ell$ is a small constant. This limits the completeness of the technique to systems with specific array, ring, and tree like topologies. [19] provides a global proof using several local proofs. It splits a target system invariant into local process invariants across local and shared variables and attempts to prove these invariants. The derived local invariants are symbolic over-approximations of the reachable state space of the system. The abstraction refinement step refines the invariants with predicates reasoning about additional local variables.

Pnueli et al. [28] targets a specific type of bounded data parameterized systems with parameter $N$, where $N$ is the number of processes, and where safety is expressed using a specific type of assertions called $R$-assertions. They show that for a given such system, there exists an $N_{0}$ such that, an $R$-assertion $\phi$ is preserved by any step of the system for every $N>1$ iff $\phi$ is preserved by any step of the system for every $N \leq N_{0}$. They show how to handle such systems with model checking and deductive reasoning techniques. The survey paper [18] describes several abstraction techniques that use counterexamples to guide the refinement steps. It also describes a localization reduction technique [22]. The first abstraction is the property itself. If the model check fails, then an error "track" is produced, and either the track is feasible and the property fails, or the track is analyzed and linked to a group of blocking variables that could not be assigned to satisfy the track. The blocking variables lead, via dependency graph paths, to active variables that have full assignments in the error track. All these variables constitute the next refinement step, where the border variables are considered free and are called the "free fence". Key to the efficacy of the technique is the choice of the blocking variables, so that they minimize the free fence at each abstraction step. Localization refinement is also used synergistically with input re-parameterization, to attain maximal input reduction in sequential netlists, using min-cut analysis in a structural manner [12]. The reduced netlists are then subject to verification using several techniques, including decomposing the netlist into several sub-netlists, each with a bounded state transition diameter, and then applying bounded model checking $[10,11]$ to each sub-netlist. Our work differs from the above techniques in that (1) we do not limit our technique to parameterized systems, (2) we characterize deadlock freedom with a structural supercycle property that governs the wait-for-graph of the system interactions, (3) we compute our local subsystems based on interactions, (4) we establish deadlock freedom by performing the structural supercycle violation check for each interaction using its local subsystems, and (5) our technique is complete for BIP systems. In the future, we would like to explore whether the local supercycle violation check is enough to prove deadlock-freedom of parameterized systems. 
We would also like to consider characterizing other interesting safety properties with similar structural checks in the context of BIP.

\subsection{Discussion}

Our approach has the following advantages:

- Local and global deadlock: our method shows that no subset of processes can be deadlocked, i.e., absence of both local and global deadlock.

- Check works for realistic formalism: by applying the approach to BIP, we provide an efficient deadlock-freedom check within a formalism from which efficient distributed implementations can be generated [15].

- Locality: if a component $\mathrm{B}_{i}$ is modified, or is added to an existing system, then $\mathcal{L} \mathcal{A} \mathcal{L} \mathcal{T}\left(\mathrm{B}, Q_{0}, \mathrm{a}, \ell\right)$ only has to be re-checked for $\mathrm{B}_{i}$ and components within distance $\ell$ of $B_{i}$. A condition whose evaluation considers the entire system at once, e.g., [2,13,21] would have to be re-checked for the entire system.

- Easily parallelizable: since the checking of each subsystem D is independent of the others, the checks can be carried out in parallel. Hence our method can be easily parallelized and distributed, for speedup, if needed. Alternatively, performing the checks sequentially minimizes the amount of memory needed.

- Framework aspect: supercycles and in/out-depth provide a framework for deadlock-freedom. Conditions more general and/or discriminating than the one presented here should be devisable in this framework. This is a topic for future work. In addition, our approach is applicable to any model of concurrency in which our notions of wait-for graph and supercycle can be defined. For example, [7] give two methods for verifying global and local deadlock freedom of shared-memory concurrent programs in pairwise normal form, as noted above. Hence, our methods are applicable to other formalisms such as CSP, CCS, I/O Automata, etc.

\subsection{Further work}

Our implementation uses explicit state enumeration. Using BDD's may improve the running time when $\mathcal{L} \mathcal{A} \mathcal{L} \mathcal{T}\left(\mathrm{B}, Q_{0}, \mathrm{a}, \ell\right)$ holds only for large $\ell$. Another potential method for improving the running time is to use SAT solving, cf. [3]. An enabled port $p$ enables all interactions containing $p$. Deadlock-freedom conditions based on ports could exploit this interdependence among interaction enablement. Our implementation should produce counterexamples when a system fails to satisfy $\mathcal{L} \mathcal{A} \mathcal{L} \mathcal{T}\left(\mathrm{B}, Q_{0}, \mathrm{a}, \ell\right)$. These can be used to manually modify the system to eliminate a possible deadlock. Also, when $\mathcal{L} \mathcal{A} \mathcal{L} \mathcal{T}\left(\mathrm{B}, Q_{0}\right.$, a,$\left.\ell\right)$ fails to verify deadlock-freedom, we increment $\ell$, in effect extending the subsystem being checked "in all directions" away from $a$ (in the structure graph). A counterexample may provide guidance to a more discriminating extension, when adds only a few components, so we now consider subsystems whose boundary has varying distance from $a$, in the structure graph. This has the benefit that we might verify deadlock freedom using a smaller subsystem than with our current approach. Design rules for ensuring $\mathcal{L} \mathcal{A} \mathcal{L} \mathcal{T}\left(\mathrm{B}, Q_{0}, \mathrm{a}, \ell\right)$ will help users to produce deadlock-free systems, and also to interpret counterexamples. A fault may create a deadlock, i.e., a supercycle, by creating wait-for-edges that would not normally arise. Tolerating a fault that creates up to $f$ such spurious wait-for-edges requires that there do not arise during normal (fault-free) operation subgraphs of $W_{\mathrm{B}}(s)$ that can be made into a supercycle by adding $f$ edges. We will investigate criteria for preventing formation of such subgraphs. Methods for evaluating $\mathcal{L} \mathcal{A} \mathcal{L} \mathcal{T}\left(\mathrm{B}, Q_{0}, \mathrm{a}, \ell\right)$ on infinite state systems will be devised, e.g.,, by extracting proof obligations and verifying using SMT solvers. We will extend our method to Dynamic BIP, [16], where participants can add and remove interactions at run time. 


\section{ACKNOWLEDGMENTS}

The research leading to these results has received funding from University Research Board (URB) at the American University of Beirut.

\section{REFERENCES}

[1] Parosh Aziz Abdulla, Frédéric Haziza, and Lukás Holík. 2013. All for the Price of Few. In Verification, Model Checking, and Abstract Interpretation, International Conference, VMCAI. Springer-Verlag, Rome, Italy, 476-495. https://doi.org/10. 1007/978-3-642-35873-9_28

[2] Alessandro Aldini and Marco Bernardo. 2003. A General Approach to Deadlock Freedom Verification for Software Architectures. FME 2805 (2003), 658-677.

[3] Pedro Antonino, Thomas Gibson-Robinson, and A. W. Roscoe. 2016. Efficient Deadlock-Freedom Checking Using Local Analysis and SAT Solving. In Integrated Formal Methods - 12th International Conference, IFM. Springer-Verlag, Reykjavik, Iceland, 345-360. https://doi.org/10.1007/978-3-319-33693-0_22

[4] Paul C. Attie. 2016. Finite-state concurrent programs can be expressed in pairwise normal form. Theoretical Computer Science 619 (2016), 1 - 31. https://doi.org/10.1016/j.tcs.2015.11.032

[5] Paul C. Attie. 2016. Synthesis of large dynamic concurrent programs from dynamic specifications. Formal Methods in System Design 48, 1-2 (2016), 1-54. https://doi.org/10.1007/s10703-016-0252-9

[6] Paul C. Attie, Saddek Bensalem, Marius Bozga, Mohamad Jaber, Joseph Sifakis, and Fadi A. Zaraket. 2013. An Abstract Framework for Deadlock Prevention in BIP. In Formal Techniques for Distributed Systems - foint IFIP WG 6.1 International Conference, FMOODS/FORTE 2013, Held as Part of the 8th International Federated Conference on Distributed Computing Techniques, DisCoTec 2013, Florence, Italy, fune 3-5, 2013. Proceedings. Springer-Verlag, Florence, Italy, 161-177. https://doi.org/10.1007/978-3-642-38592-6_12

[7] Paul C. Attie and Hana Chockler. 2005. Efficiently Verifiable Conditions for Deadlock-Freedom of Large Concurrent Programs.. In VMCAI (Lecture Notes in Computer Science), Radhia Cousot (Ed.), Vol. 3385. Springer, Paris, France, 465-481.

[8] Paul C. Attie and E. Allen Emerson. 1998. Synthesis of Concurrent Systems with Many Similar Processes. TOPLAS 20, 1 (Jan. 1998), 51-115.

[9] Paul C. Attie, Nissim Francez, and Orna Grumberg. 1993. Fairness and Hyperfairness in Multiparty Interactions. Distributed Computing 6 (1993), 245-254.

[10] Jason Baumgartner and Andreas Kuehlmann. 2004. Enhanced Diameter Bounding via Structural Transformation. In Design, Automation and Test in Europe Conference and Exposition DATE. IEEE, Paris, France, 36-41. https://doi.org/10. 1109/DATE.2004.1268824

[11] Jason Baumgartner, Andreas Kuehlmann, and Jacob A. Abraham. 2002. Property Checking via Structural Analysis. In Computer Aided Verification, CAV. Springer-Verlag, Copenhagen, Denmark, 151-165. https://doi.org/10.1007/ 3-540-45657-0_12

[12] Jason Baumgartner and Hari Mony. 2005. Maximal Input Reduction of Sequential Netlists via Synergistic Reparameterization and Localization Strategies. In Correct Hardware Design and Verification Methods, CHARME. Springer-Verlag, SaarbrÃijcken, Germany, 222-237. https://doi.org/10.1007/11560548_18

[13] Saddek Bensalem, Andreas Griesmayer, Axel Legay, Thanh-Hung Nguyen, Joseph Sifakis, and Rongjie Yan. 2011. D-Finder 2: Towards Efficient Correctness of Incremental Design. In NASA Formal Methods. Springer-Verlag, Pasadena, CA, USA, 453-458.

[14] Simon Bliudze and Joseph Sifakis. 2008. The Algebra of Connectors-Structuring Interaction in BIP. IEEE Trans. Comput. 57, 10 (2008), 1315-1330. https://doi.org/10.1109/TC.2008.26

[15] Borzoo Bonakdarpour, Marius Bozga, Mohamad Jaber, Jean Quilbeuf, and Joseph Sifakis. 2010. From High-level Component-based Models to Distributed Implementations. In EMSOFT. ACM, Scottsdale, Arizona, USA, 209-218.

[16] Marius Bozga, Mohamad Jaber, Nikolaos Maris, and Joseph Sifakis. 2012. Modeling Dynamic Architectures Using Dy-BIP. In Software Composition. Springer-Verlag, Prague, Czech Republic, 1-16.

[17] Stephen Brookes and Andrew William Roscoe. 1991. Deadlock analysis in networks of communicating processes. Distributed Computing 4 (1991), 209-230. Issue 4.

[18] Edmund M. Clarke, Robert P. Kurshan, and Helmut Veith. 2010. The Localization Reduction and CounterexampleGuided Abstraction Refinement. In Time for Verification, Essays in Memory of Amir Pnueli. Springer-Verlag, New York, NY, USA, 61-71. https://doi.org/10.1007/978-3-642-13754-9_4

[19] Ariel Cohen and Kedar S. Namjoshi. 2009. Local proofs for global safety properties. Formal Methods in System Design 34, 2 (5 2009), 104-125. https://doi.org/10.1007/s10703-008-0063-8

[20] Brian Davey and Hilary Priestly. 2002. Itroduction to Lattices and Order. Cambridge University Press, Cambridge, UK. 
[21] Gregor Gössler and Joseph Sifakis. 2003. Component-based Construction of Deadlock-free Systems. In FSTTCS. Springer, Mumbai, India, 420-433.

[22] Robert P. Kurshan. 1994. Computer-aided Verification of Coordinating Processes: The Automata-theoretic Approach Princeton University Press, Princeton, NJ, USA.

[23] Moritz Martens and Mila Majster-Cederbaum. 2012. Deadlock-freedom in component systems with architectural constraints. FMSD 41 (2012), 129-177. Issue 2. https://doi.org/10.1007/s10703-012-0160-6

[24] Jeremy Malcolm Randolph Martin. 1996. The Design and Construction of Deadlock-Free Concurrent Systems. Ph.D. Dissertation. The University of Buckingham.

[25] Robin Milner. 1989. Communication and concurrency. Prentice Hall, New Jersey, USA.

[26] Christos H. Papadimitriou. 1994. Computational complexity. Addison-Wesley, Boston Massachusetts, USA.

[27] David Park. 1969. Fixpoint induction and proofs of program properties. Machine Intelligence 5 (1969), 59-78.

[28] Amir Pnueli, Sitvanit Ruah, and Lenore D. Zuck. 2001. Automatic Deductive Verification with Invisible Invariants. In Tools and Algorithms for the Construction and Analysis of Systems, TACAS. Springer-Verlag, Genova, Italy, 82-97. https://doi.org/10.1007/3-540-45319-9_7

[29] Andrew William Roscoe and Naiem Dathi. 1987. The pursuit of deadlock freedom. Information and Computation 75, 3 (1987), 289 - 327. https://doi.org/10.1016/0890-5401(87)90004-6

[30] Robert Tarjan. 1972. Depth-First Search and Linear Graph Algorithms. SIAM J. Comput. 1, 2 (1972), 146-160 https://doi.org/10.1137/0201010

[31] Rob J. van Glabbeek, Bas Luttik, and Nikola Trcka. 2009. Computation Tree Logic with Deadlock Detection. Logical Methods in Computer Science 5, 4 (10 2009), 1-24.

Received February 2007; revised March 2009; accepted June 2009 